\title{
Regiodivergent Synthesis of Butenolide-Based $\alpha$ - and $\beta$-Amino Acid Derivatives via Base-Controlled Azirine Ring Expansion
}

Pavel A. Sakharov, Nikolai V. Rostovskii, Alexander F. Khlebnikov, and Mikhail S. Novikov*

${ }^{\dagger}$ Saint Petersburg State University, Institute of Chemistry, 7/9 Universitetskaya nab., Saint Petersburg 199034, Russia

*E-mail: m.novikov@spbu.ru

\section{Table of Contents}

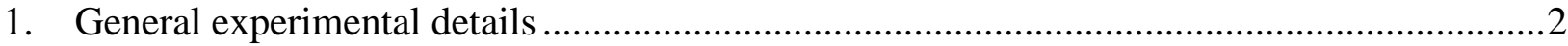

2. Optimization of the reaction of azirine $\mathbf{1 a}$ with acid $\mathbf{2 a}$.....................................................

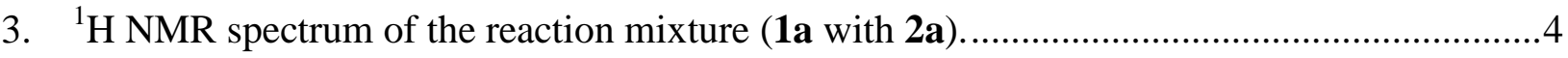

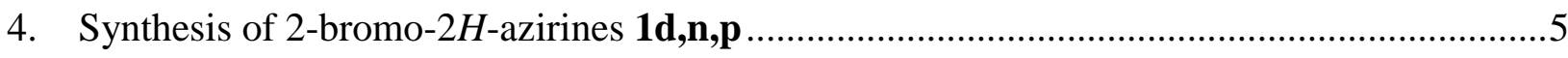

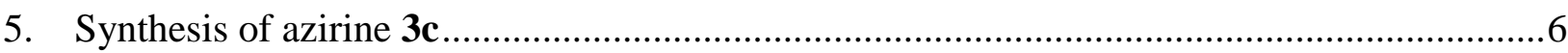

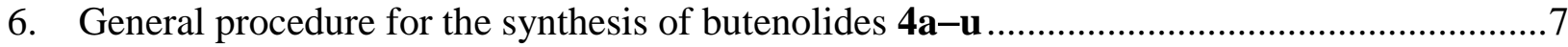

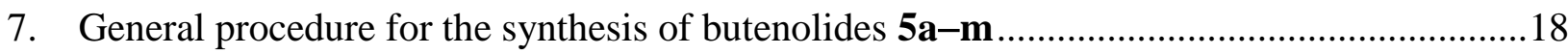

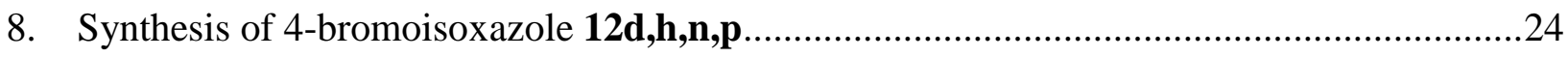

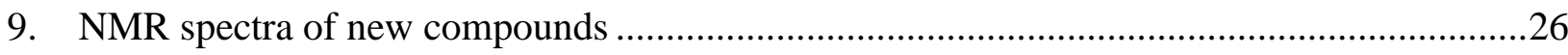

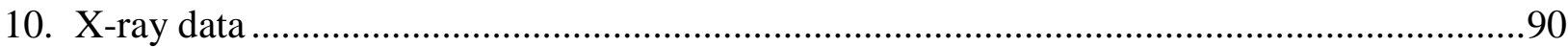

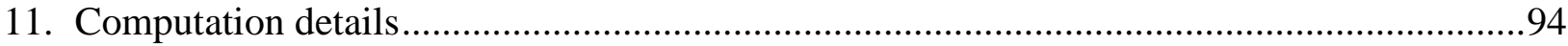

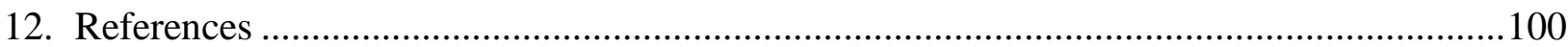




\section{General experimental details}

Melting points were determined on a melting point apparatus and are uncorrected. ${ }^{1} \mathrm{H}(400 \mathrm{MHz})$ and ${ }^{13} \mathrm{C}(100 \mathrm{MHz})$ NMR spectra are reported in parts per million downfield from tetramethylsilane. High-resolution mass spectra were recorded on an HRMS-ESI-QTOF instrument, electrospray ionization. Single crystal X-ray data were collected by means of diffractometer at $100 \mathrm{~K}$ using monochromated $\mathrm{CuK \alpha}$ (for 5a) and $\mathrm{MoK} \alpha$ (for 4o) radiation. Crystallographic data for the structures 4o (CCDC 1902292) and 5a (CCDC 1986590) have been deposited with the Cambridge Crystallographic Data Centre. Thin-layer chromatography (TLC) was conducted on aluminum sheets precoated with $\mathrm{SiO}_{2}$ ALUGRAM SIL G/UV254. All solvents were distilled and dried prior to use. Acetonitrile distilled from $\mathrm{P}_{2} \mathrm{O}_{5}$, then distilled from anhydrous $\mathrm{K}_{2} \mathrm{CO}_{3}$ and stored over anhydrous $\mathrm{K}_{2} \mathrm{CO}_{3}$.

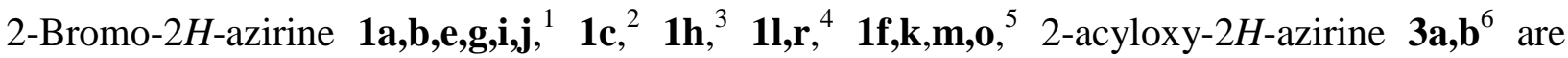
known compounds, which were prepared by the reported procedures. 
2. Table S1. Optimization of the reaction of azirine $\mathbf{1 a}$ with acid $\mathbf{2} \mathbf{a}^{\mathbf{a}}$

\begin{tabular}{|c|c|c|c|c|c|c|c|c|}
\hline \multirow[b]{2}{*}{ entry } & \multirow[b]{2}{*}{$\mathrm{X}$} & $e^{P N}$ & $\mathrm{CO}_{2} \mathrm{Y}$ & ${ }_{\mathrm{O}}^{\mathrm{PNP}} \underbrace{\mathrm{Ph}}_{3 \mathbf{a}}$ & PNP & PN & $=\sum_{5 \mathrm{Ph}}^{\mathrm{CO}_{2} \mathrm{~N}}$ & \\
\hline & & $\mathrm{Y}$ & base & additive & solvent & $\begin{array}{l}\text { yield of } \\
\mathbf{3 a},{ }^{b} \%\end{array}$ & $\begin{array}{c}\text { yield of } \\
\mathbf{4 a},{ }^{b} \%\end{array}$ & $\begin{array}{l}\text { yield of } \\
\mathbf{5 a}{ }^{b} \%\end{array}$ \\
\hline 1 & $\mathrm{Br}$ & $\mathrm{H}$ & $\mathrm{Et}_{3} \mathrm{~N}$ & - & toluene & 98 & 0 & 0 \\
\hline 2 & $\mathrm{Br}$ & $\mathrm{H}$ & $\mathrm{Et}_{3} \mathrm{~N}$ & - & $\mathrm{MeOH}$ & 0 & 0 & 0 \\
\hline 3 & $\mathrm{Br}$ & $\mathrm{H}$ & $\mathrm{Et}_{3} \mathrm{~N}$ & - & $\mathrm{CHCl}_{3}$ & 80 & 0 & 0 \\
\hline 4 & $\mathrm{Br}$ & $\mathrm{H}$ & $\mathrm{Et}_{3} \mathrm{~N}$ & - & $\mathrm{MeCN}$ & 0 & 13 & 76 \\
\hline 5 & $\mathrm{Br}$ & $\mathrm{H}$ & DIPEA & - & $\mathrm{MeCN}$ & 0 & 33 & 54 \\
\hline 6 & $\mathrm{Br}$ & $\mathrm{H}$ & $\mathrm{K}_{2} \mathrm{CO}_{3}$ & - & $\mathrm{MeCN}$ & 0 & 85 & 10 \\
\hline $7^{c}$ & $\mathrm{Br}$ & $\mathrm{H}$ & $\mathrm{Li}_{2} \mathrm{CO}_{3}$ & - & $\mathrm{MeCN}$ & 85 & 0 & 0 \\
\hline $8^{c}$ & $\mathrm{Br}$ & $\mathrm{H}$ & $\mathrm{Na}_{2} \mathrm{CO}_{3}$ & - & $\mathrm{MeCN}$ & 0 & 74 & 16 \\
\hline $9^{c}$ & $\mathrm{Br}$ & $\mathrm{H}$ & $\mathrm{Cs}_{2} \mathrm{CO}_{3}$ & - & $\mathrm{MeCN}$ & 0 & 59 & 33 \\
\hline 10 & I & $\mathrm{H}$ & $\mathrm{Et}_{3} \mathrm{~N}$ & - & $\mathrm{MeCN}$ & 0 & 18 & 56 \\
\hline $11^{d}$ & $4-\mathrm{NO}_{2} \mathrm{C}_{6} \mathrm{H}_{4} \mathrm{CO}_{2}$ & $\mathrm{H}$ & $\mathrm{Et}_{3} \mathrm{~N}$ & - & $\mathrm{MeCN}$ & 0 & 14 & 60 \\
\hline 12 & $\mathrm{Br}$ & $\mathrm{K}^{e}$ & - & - & $\mathrm{MeCN}$ & 0 & 45 & 45 \\
\hline 13 & $\mathrm{Br}$ & $\mathrm{K}$ & $\mathrm{Et}_{3} \mathrm{~N}$ & - & $\mathrm{MeCN}$ & 0 & 26 & 64 \\
\hline 14 & $\mathrm{Br}$ & $\mathrm{K}^{e}$ & - & $\mathrm{Bu}_{4} \mathrm{NBr}$ & $\mathrm{MeCN}$ & 0 & 34 & 51 \\
\hline 15 & $\mathrm{Br}$ & $\mathrm{K}^{f}$ & - & 18-crown-6 & $\mathrm{MeCN}$ & 0 & 33 & 47 \\
\hline \multicolumn{9}{|c|}{${ }^{a}$ Reaction conditions: azirine $1(0.08 \mathrm{mmol}), \mathrm{RCO}_{2} \mathrm{Y}(0.088 \mathrm{mmol})$, base $(0.16 \mathrm{mmol})$, solvent $(1.5 \mathrm{ml}), \mathrm{rt}, 24 \mathrm{~h}}$. \\
\hline \multicolumn{9}{|c|}{${ }^{c}$ For $96 \mathrm{~h}}$. \\
\hline \multicolumn{9}{|c|}{${ }^{d}$ For $14 \mathrm{~d}$} \\
\hline \multicolumn{9}{|c|}{${ }^{\mathrm{e}}$ The 2 equiv of the salt was used. } \\
\hline \multicolumn{9}{|c|}{$\begin{array}{l}{ }^{f} \text { Potassium salt of 4-nitrophenylacetic acid }(0.16 \mathrm{mmol}) \text { was added to a solution of 2-bromo- } 2 \mathrm{H} \text {-azirine } \mathbf{1 a}(0.08 \\
\mathrm{mmol}) \text { and } 18 \text {-crown- } 6(0.2 \mathrm{mmol}) \text { in } \mathrm{MeCN}(2 \mathrm{~mL}) \text { at } \mathrm{rt} \text {, and the reaction mixture was stirred for } 2 \mathrm{~h} \text {. All volatiles } \\
\text { were removed in vacuo. } \mathrm{CDCl}_{3}(0.5 \mathrm{~mL}) \text { and } \mathrm{CH}_{2} \mathrm{Br}_{2}(15 \mathrm{mg}) \text { were added, and the mixture was analyzed by }{ }^{1} \mathrm{H} \\
\text { NMR spectroscopy. }\end{array}$} \\
\hline
\end{tabular}


3. Figure S1. ${ }^{1} \mathrm{H}$ NMR spectrum of the reaction mixture $[\mathbf{1 a}(0.039 \mathrm{mmol}), \mathbf{2 a}$ (0.045 mmol), DIPEA $(0.07 \mathrm{mmol})$ in $\left.\mathrm{MeCN}^{-\mathrm{d}_{3}}(0.5 \mathrm{~mL}), \mathrm{rt}\right] 8 \mathrm{~min}$ after mixing the reagents $(400 \mathrm{MHz})$.

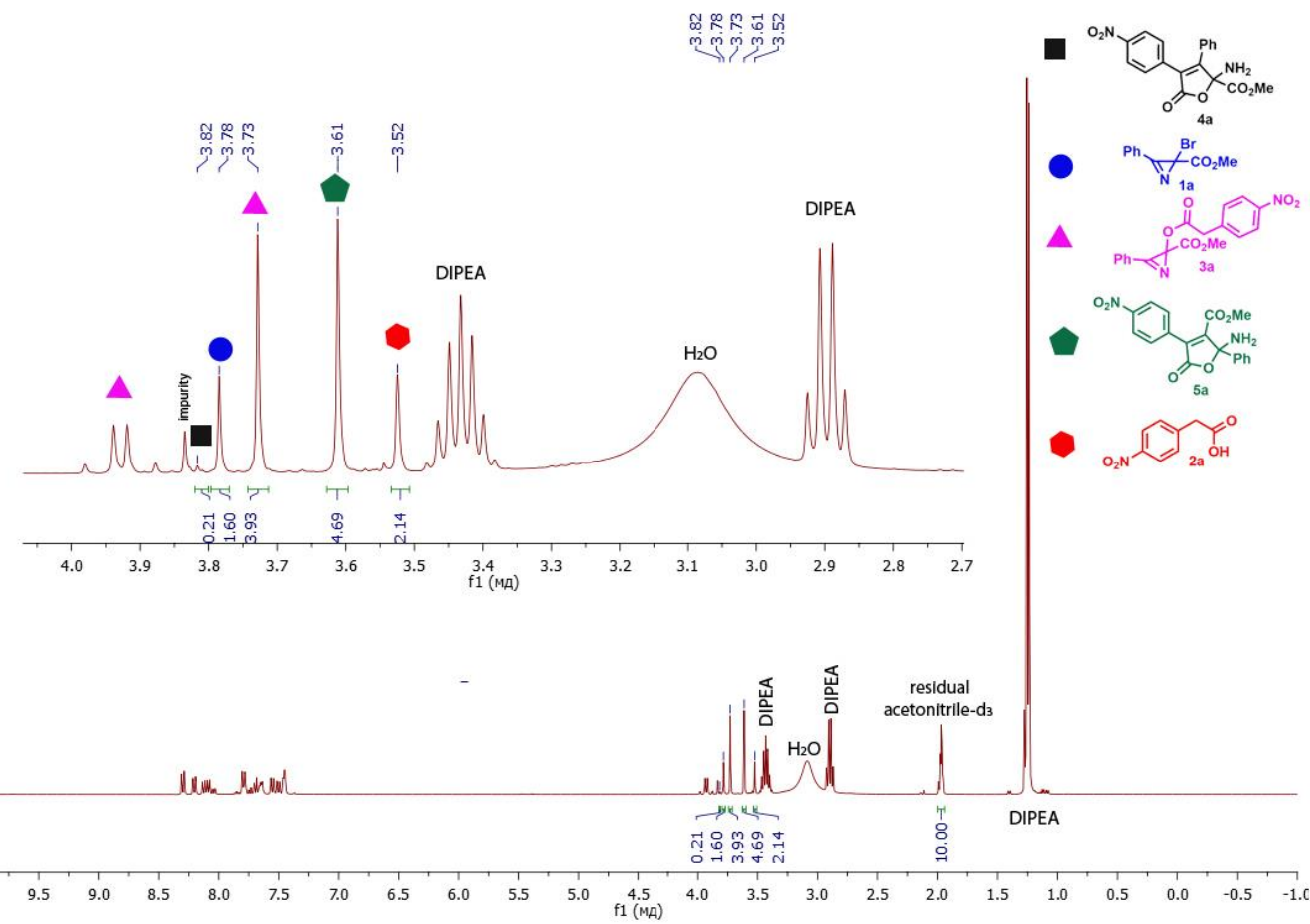




\section{Synthesis of 2-bromo-2H-azirines 1d,n,p}

2-Bromo-2 $H$-azirines 1d,n,p were synthesized from 4-bromoisoxazoles 12d,n,p and $\mathrm{FeCl}_{2} \cdot 4 \mathrm{H}_{2} \mathrm{O}$ according to the reported procedure ${ }^{1}$.

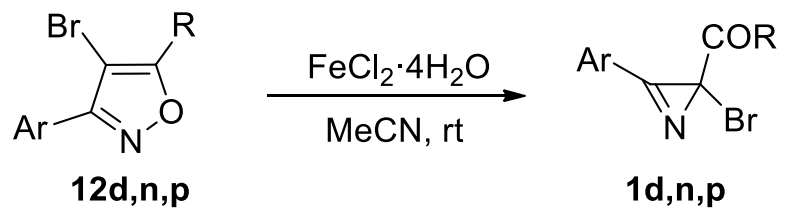

Methyl 2-bromo-3-(4-fluorophenyl)-2H-azirine-2-carboxylate (1d)<smiles>CC(=O)C1(Br)N=C1c1ccc(F)cc1</smiles>

Colorless solid (442 mg, 95\%); mp 75-76 ${ }^{\circ} \mathrm{C}\left(\mathrm{Et}_{2} \mathrm{O} /\right.$ hexane). ${ }^{1} \mathrm{H}$ NMR (400 MHz, $\left.\mathrm{CDCl}_{3}\right) \delta 3.84$ (s, 3H), 7.33-7.37 (m, 2H), 7.99-8.02 (m, 2H); $\left.{ }^{13} \mathrm{C} \mathrm{NMR} \mathrm{(100} \mathrm{MHz,} \mathrm{CDCl}_{3}\right) \delta$ 43.7, 54.2, 116.1 $(\mathrm{d}, J=3.1 \mathrm{~Hz}), 117.4(\mathrm{~d}, J=22.7 \mathrm{~Hz}), 133.6(\mathrm{~d}, J=9.9 \mathrm{~Hz}), 163.5,166.8(\mathrm{~d}, J=259.6 \mathrm{~Hz})$, 167.1; HRMS (ESI) m/z: $[\mathrm{M}+\mathrm{Na}]^{+}$Calcd for $\mathrm{C}_{10} \mathrm{H}_{7}{ }^{79} \mathrm{BrFNNaO}_{2}{ }^{+}$293.9536; Found 293.9538.

\section{$N$-Benzyl-2-bromo- $N$-methyl-3-phenyl-2H-azirine-2-carboxamide (1n)}<smiles>CN(Cc1ccccc1)C(=O)C1(Br)N=C1c1ccccc1</smiles>

Colorless oil (398 mg, 88\%). $1: 1.75$ mixture of rotamers. ${ }^{1} \mathrm{H}$ NMR $\left(400 \mathrm{MHz}, \mathrm{CDCl}_{3}\right) \delta 2.85$ $(\mathrm{s}, 3 \mathrm{H}), 3.52(\mathrm{~s}, 5.25 \mathrm{H}), 4.52(\mathrm{~d}, J=14.7 \mathrm{~Hz}, 1.75 \mathrm{H}), 4.71(\mathrm{~d}, J=14.7 \mathrm{~Hz}, 1.75 \mathrm{H}), 5.09(\mathrm{~d}, J=$ $15.4 \mathrm{~Hz}, 1 \mathrm{H}), 5.45(\mathrm{~d}, J=15.4 \mathrm{~Hz}, 1 \mathrm{H}), 7.27-7.40(\mathrm{~m}, 13.75 \mathrm{H}), 7.63-7.67(\mathrm{~m}, 8.25 \mathrm{H}), 8.19(\mathrm{~d}, J$ $=7.8 \mathrm{~Hz}, 5.5 \mathrm{H}) ;{ }^{13} \mathrm{C} \mathrm{NMR}\left(100 \mathrm{MHz}, \mathrm{CDCl}_{3}\right) \delta 32.3,35.3,47.7,48.3,50.8,53.9,120.7$ (2C), 127.5, 127.8, 127.9 (2C), 128.7 (2C), 129.5 (2C), 130.3, 131.3 (2C), 135.1, 135.7, 136.1, 164.6, 164.8, 172.4, 172.7; HRMS (ESI) m/z: $[\mathrm{M}+\mathrm{Na}]^{+} \mathrm{Calcd}$ for $\mathrm{C}_{17} \mathrm{H}_{15}{ }^{79} \mathrm{BrN}_{2} \mathrm{NaO}^{+} 365.0260$; Found 365.0252 .

tert-Butyl 2-bromo-3-phenyl-2H-azirine-2-carboxylate (1p)<smiles>CCOC(=O)C1(Br)N=C1c1ccccc1</smiles>

Yellow oil (1.1 g, 90\%). ${ }^{1} \mathrm{H}$ NMR (400 MHz, $\left.\mathrm{CDCl}_{3}\right) \delta 1.48(\mathrm{~s}, 9 \mathrm{H}), 7.63-7.67(\mathrm{~m}, 2 \mathrm{H}), 7.72-$ $7.76(\mathrm{~m}, 1 \mathrm{H}), 7.95-7.97(\mathrm{~m}, 2 \mathrm{H}) ;{ }^{13} \mathrm{C} \mathrm{NMR}\left(100 \mathrm{MHz}, \mathrm{CDCl}_{3}\right) \delta 27.7,45.8,84.1,120.1,129.6$, 130.7, 134.9, 165.0, 165.4; HRMS (ESI) m/z: $[\mathrm{M}+\mathrm{Na}]^{+}$Calcd for $\mathrm{C}_{13} \mathrm{H}_{14}{ }^{79} \mathrm{BrNNaO}_{2}{ }^{+} 318.0100$; Found 318.0094. 


\section{Synthesis of azirine $3 \mathrm{c}$}

3-Phenyl-2-(pyrrolidine-1-carbonyl)-2H-azirin-2-yl 2-(4-nitrophenyl)acetate (3c) was prepared from azirine $\mathbf{1 m}$, acid $\mathbf{2 a}$, and $\mathrm{Et}_{3} \mathrm{~N}$ according to the reported procedure 6 .
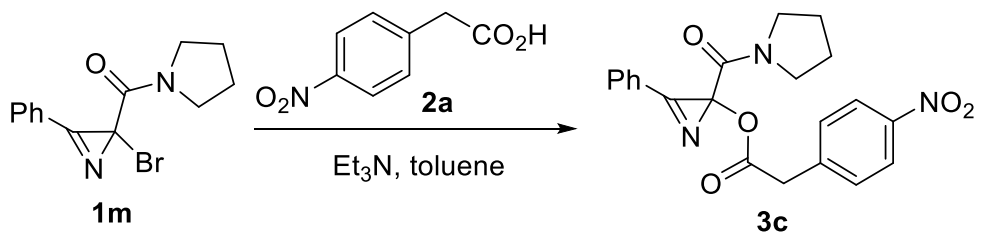

Colorless oil (76 mg, 82\%). ${ }^{1} \mathrm{H}$ NMR (400 MHz, $\left.\mathrm{CDCl}_{3}\right) \delta 1.75-1.87$ (m, 4H), 3.45-3.51 (m, $4 \mathrm{H}), 3.79$ and $3.84(\mathrm{AB}-\mathrm{q}, J=16.4 \mathrm{~Hz}, 2 \mathrm{H}), 7.45(\mathrm{~d}, J=8.6 \mathrm{~Hz}, 2 \mathrm{H}), 7.55-7.59(\mathrm{~m}, 2 \mathrm{H}), 7.64$ $7.68(\mathrm{~m}, 1 \mathrm{H}), 8.07-8.09(\mathrm{~m}, 2 \mathrm{H}), 8.14(\mathrm{~d}, J=8.6 \mathrm{~Hz}, 2 \mathrm{H}),{ }^{13} \mathrm{C} \mathrm{NMR}\left(100 \mathrm{MHz}, \mathrm{CDCl}_{3}\right) \delta 23.4$, 26.4, 40.2, 46.8, 47.1, 66.3, 121.7, 123.6, 129.2, 130.3, 130.8, 134.4, 140.2, 147.1, 163.9, 169.1, 169.5; HRMS (ESI) m/z: [M+Na] ${ }^{+}$Calcd for $\mathrm{C}_{21} \mathrm{H}_{19} \mathrm{~N}_{3} \mathrm{NaO}_{5}{ }^{+} 416.1217$; Found 416.1208. 


\section{General procedure for the synthesis of 2-amino-5-oxo-2,5-dihydrofuran-2- carboxylates $4 \mathbf{a}-\mathbf{u}$}

\section{Method A (from 2-bromoazirines)}

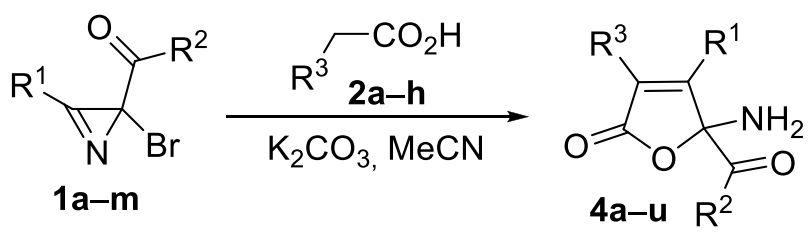

Anhydrous $\mathrm{K}_{2} \mathrm{CO}_{3}(65 \mathrm{mg}, 0.47 \mathrm{mmol})$ was added to a solution of 2-bromo- $2 \mathrm{H}$-azirine $1(0.24$ $\mathrm{mmol})$ and acid $2(0.26 \mathrm{mmol})$ in $\mathrm{MeCN}(4 \mathrm{~mL})$ at $\mathrm{rt}$, and the reaction mixture was stirred for 24 to $140 \mathrm{~h}$ as indicated below. The resulting mixture was filtered through celite using EtOAc (10 $\mathrm{mL}$ ) to completely wash away the product. The combined filtrate was concentrated in vacuo, and the product was purified by a silica gel column chromatography followed by recrystallization.

\section{Method B (from 2-acyloxyazirines)}

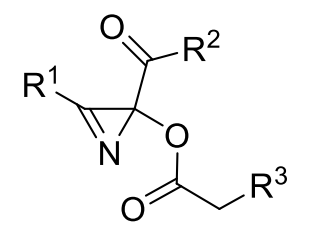

$3 a-c$

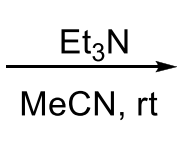

$\mathrm{Et}_{3} \mathrm{~N}(0.24 \mathrm{mmol})$ was added to a solution of acyloxyazirine $3(0.24 \mathrm{mmol})$ in $\mathrm{MeCN}(4 \mathrm{~mL})$ at $\mathrm{rt}$, and the reaction mixture was stirred for 24 to $140 \mathrm{~h}$ as indicated below. The solvent was removed in vacuo and the product was purified by a silica gel column chromatography followed by recrystallization.

Methyl 2-amino-4-(4-nitrophenyl)-5-oxo-3-phenyl-2,5-dihydrofuran-2-carboxylate (4a)<smiles>CC(=O)C1(N)OC(=O)C(c2ccc([N+](=O)[O-])cc2)=C1c1ccccc1</smiles>

Compound 4a was obtained from azirine 1a and acid 2a according to method A (67 mg, 80\%, reaction time $24 \mathrm{~h}$ ); column chromatography (petroleum ether/EtOAc $3: 1$ ). Compound 4a was also obtained according to method $\mathrm{B}$ from azirine $3 \mathbf{a}$ and $\mathrm{Et}_{3} \mathrm{~N}$ in the $0.24 \mathrm{mmol}$ scale reaction (78 $\mathrm{mg}, 92 \%$ on two steps, reaction time $24 \mathrm{~h}$ ) and in the $1 \mathrm{mmol}$ scale reaction $(322 \mathrm{mg}$, $91 \%$ on two steps). Colorless solid; mp $127-129{ }^{\circ} \mathrm{C}\left(\mathrm{Et}_{2} \mathrm{O} /\right.$ hexane). ${ }^{1} \mathrm{H}$ NMR $\left(400 \mathrm{MHz}, \mathrm{CDCl}_{3}\right) \delta 2.91$ (s, 2H), $3.85(\mathrm{~s}, 3 \mathrm{H}), 7.33-7.38(\mathrm{~m}, 4 \mathrm{H}), 7.41-7.45(\mathrm{~m}, 1 \mathrm{H}), 7.64-7.66(\mathrm{~m}, 2 \mathrm{H}), 8.18-8.20(\mathrm{~m}$, 
$2 \mathrm{H}) ;{ }^{13} \mathrm{C}$ NMR $\left(100 \mathrm{MHz}, \mathrm{CDCl}_{3}\right) \delta 54.0,94.6,123.6,127.8,128.9,129.0,129.5,130.6,130.9$, 136.1, 147.9, 157.7, 166.4, 169.2; ${ }^{15} \mathrm{~N}$ NMR from $\mathrm{HMBC}{ }^{1} \mathrm{H}-{ }^{15} \mathrm{~N}\left(400 \mathrm{MHz}, \mathrm{CDCl}_{3}\right) \delta{ }^{15} \mathrm{~N} 42.8$, 382.4; HRMS (ESI) m/z: [M+Na] $]^{+}$Calcd for $\mathrm{C}_{18} \mathrm{H}_{14} \mathrm{~N}_{2} \mathrm{NaO}_{6}{ }^{+}$377.0744; Found 377.0755.

Methyl 2-amino-3-(4-methylphenyl)-4-(4-nitrophenyl)-5-oxo-2,5-dihydrofuran-2carboxylate (4b)

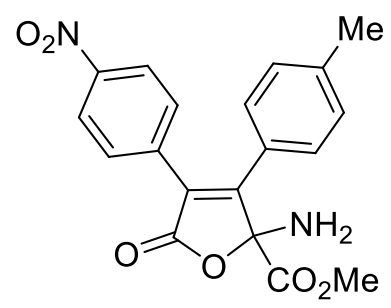

Compound 4b was obtained from azirine 1b and acid 2a according to method A (63 mg, 72\%, reaction time $24 \mathrm{~h}$ ); column chromatography (petroleum ether/EtOAc $3: 1$ ). Colorless solid; mp $192-193{ }^{\circ} \mathrm{C}\left(\mathrm{Et}_{2} \mathrm{O} /\right.$ hexane). ${ }^{1} \mathrm{H}$ NMR $\left(400 \mathrm{MHz}, \mathrm{CDCl}_{3}-\mathrm{DMSO}-d_{6}\right.$ mixture) $\delta 2.28(\mathrm{~s}, 3 \mathrm{H}), 3.15$ $(\mathrm{s}, 2 \mathrm{H}), 3.74(\mathrm{~s}, 3 \mathrm{H}), 7.06(\mathrm{~d}, J=8.0 \mathrm{~Hz}, 2 \mathrm{H}), 7.20(\mathrm{~d}, J=8.0 \mathrm{~Hz}, 2 \mathrm{H}), 7.58(\mathrm{~d}, J=8.7 \mathrm{~Hz}, 2 \mathrm{H})$, $8.12(\mathrm{~d}, J=8.7 \mathrm{~Hz}, 2 \mathrm{H}) ;{ }^{13} \mathrm{C}$ NMR $\left(100 \mathrm{MHz}, \mathrm{CDCl}_{3}-\mathrm{DMSO}-d_{6}\right.$ mixture $) \delta 20.8,53.3$, 94.6, 123.0, 126.1, 126.3, 128.4, 129.0, 130.1, 136.2, 140.7, 147.1, 157.4, 166.1, 169.0; HRMS (ESI) $\mathrm{m} / \mathrm{z}:[\mathrm{M}+\mathrm{Na}]^{+}$Calcd for $\mathrm{C}_{19} \mathrm{H}_{16} \mathrm{~N}_{2} \mathrm{NaO}_{6}{ }^{+} 391.0901$; Found 391.0893 .

Methyl 2-amino-3-(4-(tert-butyl)phenyl)-4-(4-nitrophenyl)-5-oxo-2,5-dihydrofuran-2carboxylate (4c)

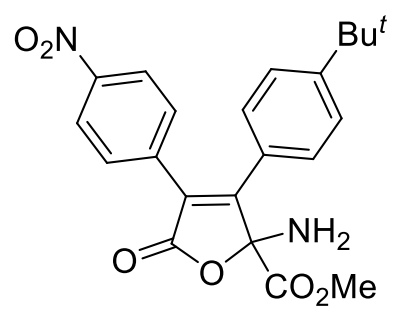

Compound $\mathbf{4 c}$ was obtained from azirine 1c and acid $\mathbf{2 a}$ according to method A (59 mg, 61\%, reaction time $48 \mathrm{~h}$ ); column chromatography (petroleum ether/EtOAc $3: 1$ ). Colorless solid; mp $173-174{ }^{\circ} \mathrm{C}\left(\mathrm{Et}_{2} \mathrm{O} /\right.$ hexane). ${ }^{1} \mathrm{H} \mathrm{NMR}\left(400 \mathrm{MHz}, \mathrm{CDCl}_{3}\right) \delta 1.31(\mathrm{~s}, 9 \mathrm{H}), 2.90(\mathrm{~s}, 2 \mathrm{H}), 3.86(\mathrm{~s}$, $3 \mathrm{H}), 7.29(\mathrm{~d}, J=8.7 \mathrm{~Hz}, 2 \mathrm{H}), 7.36(\mathrm{~d}, J=8.7 \mathrm{~Hz}, 2 \mathrm{H}), 7.67(\mathrm{~d}, J=8.8 \mathrm{~Hz}, 2 \mathrm{H}), 8.22(\mathrm{~d}, J=8.8$ $\mathrm{Hz}, 2 \mathrm{H}) ;{ }^{13} \mathrm{C} \mathrm{NMR}\left(100 \mathrm{MHz}, \mathrm{CDCl}_{3}\right) \delta 30.9,34.9,54.0,94.5,123.7,126.0,126.2,127.0,128.9$, 130.6, 136.7, 147.9, 154.7, 157.4, 166.7, 169.4, HRMS (ESI) m/z: [M-H] Calcd for $\mathrm{C}_{22} \mathrm{H}_{21} \mathrm{~N}_{2} \mathrm{O}_{6}{ }^{-}$ 409.1405; Found 409.1401.

Methyl 2-amino-3-(4-fluorophenyl)-4-(4-nitrophenyl)-5-oxo-2,5-dihydrofuran-2carboxylate (4d) 


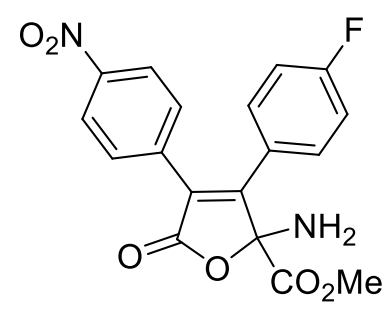

Compound 4d was obtained from azirine 1d and acid 2a according to method A (78 mg, 88\%, reaction time $48 \mathrm{~h}$ ); column chromatography (petroleum ether/EtOAc $3: 1$ ). Colorless solid; mp 169-170 ${ }^{\circ} \mathrm{C}$ (Et $2 \mathrm{O} /$ hexane). ${ }^{1} \mathrm{H}$ NMR (400 MHz, $\mathrm{CDCl}_{3}-\mathrm{DMSO}_{6} \mathrm{~d}_{6}$ mixture) $\delta 3.75(\mathrm{~s}, 3 \mathrm{H}), 3.96$ $(\mathrm{s}, 2 \mathrm{H}), 7.12-7.14(\mathrm{~m}, 2 \mathrm{H}), 7.41-7.45(\mathrm{~m}, 2 \mathrm{H}), 7.60(\mathrm{~d}, J=8.9 \mathrm{~Hz}, 2 \mathrm{H}), 8.23(\mathrm{~d}, J=8.9 \mathrm{~Hz}$, $2 \mathrm{H}) ;{ }^{13} \mathrm{C}$ NMR (100 MHz, $\mathrm{CDCl}_{3}-\mathrm{DMSO}_{-} \mathrm{d}_{6}$ mixture) $\delta 53.8,96.7,116.1$ (d, $\left.J=21.8 \mathrm{~Hz}\right), 123.9$, $126.7(\mathrm{~d}, J=3.5 \mathrm{~Hz}), 127.8,130.9,131.8(\mathrm{~d}, J=8.6 \mathrm{~Hz}), 136.9,147.8,157.0,163.3(\mathrm{~d}, J=$ $250.2 \mathrm{~Hz}$ ), 166.7, 169.5; HRMS (ESI) m/z: [M-H] Calcd for $\mathrm{C}_{18} \mathrm{H}_{12} \mathrm{FN}_{2} \mathrm{O}_{6}{ }^{-}$371.0685; Found 371.0675 .

Methyl 2-amino-3-(4-chlorophenyl)-4-(4-nitrophenyl)-5-oxo-2,5-dihydrofuran-2carboxylate (4e)

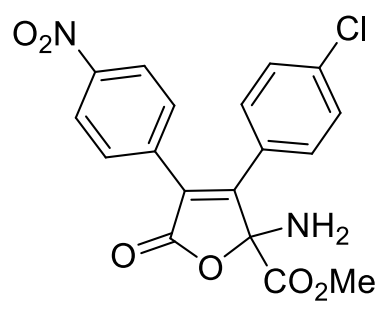

Compound 4e was obtained from azirine 1e and acid $\mathbf{2 a}$ according to method A (85 mg, 92\%, reaction time $48 \mathrm{~h}$ ); column chromatography (petroleum ether/EtOAc $3: 1$ ). Colorless solid; mp 182-183 ${ }^{\circ} \mathrm{C}$ (Et $2 \mathrm{O} /$ hexane). ${ }^{1} \mathrm{H}$ NMR (400 MHz, $\mathrm{CDCl}_{3}-\mathrm{DMSO}-\mathrm{d}_{6}$ mixture) $\delta 3.75$ (s, 3H), 3.98 $(\mathrm{s}, 2 \mathrm{H}), 7.39(\mathrm{~s}, 4 \mathrm{H}), 7.60(\mathrm{~d}, J=8.9 \mathrm{~Hz}, 2 \mathrm{H}), 8.24(\mathrm{~d}, J=8.9 \mathrm{~Hz}, 2 \mathrm{H}) ;{ }^{13} \mathrm{C} \mathrm{NMR}(100 \mathrm{MHz}$, $\mathrm{CDCl}_{3}-\mathrm{DMSO}_{6} \mathrm{~d}_{6}$ mixture) $\delta 53.9,96.7,124.0,128.3,129.1,129.2,131.0(2 \mathrm{C}), 135.6,136.7$, 147.8, 156.8, 166.6, 169.4; HRMS (ESI) m/z: [M-H] $]^{-}$Calcd for $\mathrm{C}_{18} \mathrm{H}_{12}{ }^{35} \mathrm{ClN}_{2} \mathrm{O}_{6}{ }^{-} 387.0389$; Found 387.0379 .

Methyl 2-amino-3-(4-bromophenyl)-4-(4-nitrophenyl)-5-oxo-2,5-dihydrofuran-2carboxylate (4f)<smiles>CC(=O)C1(N)OC(=O)C(c2ccc([N+](=O)[O-])cc2)=C1c1ccc(Br)cc1</smiles> 
Compound $4 \mathbf{f}$ was obtained from azirine $1 \mathbf{f}$ and acid 2a according to method A (77 mg, 75\%, reaction time $48 \mathrm{~h}$ ); column chromatography (petroleum ether/EtOAc $3: 1$ ). Colorless solid; mp $179-180{ }^{\circ} \mathrm{C}\left(\mathrm{Et}_{2} \mathrm{O} /\right.$ hexane). ${ }^{1} \mathrm{H}$ NMR $\left(400 \mathrm{MHz}, \mathrm{CDCl}_{3}-\mathrm{DMSO}_{6} \mathrm{~d}_{6}\right.$ mixture) $\delta 3.75(\mathrm{~s}, 3 \mathrm{H}), 3.89$ (s, 2H), 7.32 (d, $J=8.2 \mathrm{~Hz}, 2 \mathrm{H}), 7.49$ (d, $J=8.2 \mathrm{~Hz}, 2 \mathrm{H}), 7.60(\mathrm{~d}, J=8.4 \mathrm{~Hz}, 2 \mathrm{H}), 8.21(\mathrm{~d}, J=$ $8.4 \mathrm{~Hz}, 2 \mathrm{H}) ;{ }^{13} \mathrm{C}$ NMR (100 MHz, $\mathrm{CDCl}_{3}-\mathrm{DMSO}-\mathrm{d}_{6}$ mixture) $\delta 53.8,96.4,123.8,124.4,128.3$, 129.5, 130.8, 131.1, 131.9, 136.6, 147.8, 156.8, 166.5, 169.4; HRMS (ESI) m/z: [M-H] ${ }^{-}$Calcd for $\mathrm{C}_{18} \mathrm{H}_{12}{ }^{79} \mathrm{BrN}_{2} \mathrm{O}_{6}{ }^{-} 430.9884$; Found 430.9878 .

\section{Methyl 2-amino-3-(4-methoxyphenyl)-4-(4-nitrophenyl)-5-oxo-2,5-dihydrofuran-2- carboxylate $(4 \mathrm{~g})$}

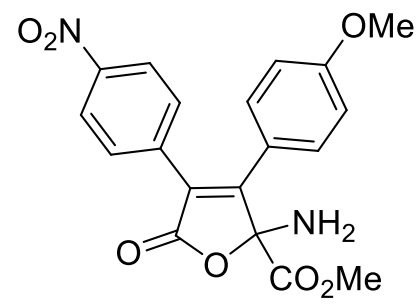

Compound $\mathbf{4 g}$ was obtained from azirine $\mathbf{1 g}$ and acid $\mathbf{2 a}$ according to method A (61 $\mathrm{mg}, 67 \%$, reaction time $48 \mathrm{~h}$ ); column chromatography (petroleum ether/EtOAc $3: 1$ ). Colorless solid; mp $138-139{ }^{\circ} \mathrm{C}\left(\mathrm{Et}_{2} \mathrm{O} / \mathrm{hexane}\right) .{ }^{1} \mathrm{H}$ NMR $\left(400 \mathrm{MHz}, \mathrm{CDCl}_{3}\right) \delta 2.90(\mathrm{~s}, 2 \mathrm{H}), 3.83(\mathrm{~s}, 6 \mathrm{H}), 6.84(\mathrm{~d}, J=$ $8.8 \mathrm{~Hz}, 2 \mathrm{H}), 7.38(\mathrm{~d}, J=8.8 \mathrm{~Hz}, 2 \mathrm{H}), 7.68(\mathrm{~d}, J=8.7 \mathrm{~Hz}, 2 \mathrm{H}), 8.22(\mathrm{~d}, J=8.7 \mathrm{~Hz}, 2 \mathrm{H}) ;{ }^{13} \mathrm{C}$ NMR $\left(100 \mathrm{MHz}, \mathrm{CDCl}_{3}\right) \delta 53.9,55.3,94.3,114.4,121.4,123.7,125.6,130.6,131.0,136.9$, 147.8, 157.1, 161.7, 166.7, 169.5; HRMS (ESI) m/z: $[\mathrm{M}+\mathrm{H}]^{+}$Calcd for $\mathrm{C}_{19} \mathrm{H}_{17} \mathrm{~N}_{2} \mathrm{O}_{7}{ }^{+} 385.1030$; Found 385.1041.

Methyl 2-amino-3-(3,4-dimethoxyphenyl)-4-(4-nitrophenyl)-5-oxo-2,5-dihydrofuran-2carboxylate (4h)

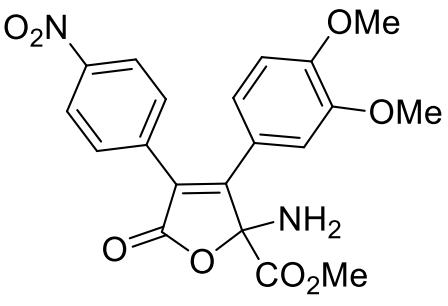

Compound 4h was obtained from azirine $\mathbf{1 h}$ and acid 2a according to method A (64 mg, 65\%, reaction time $48 \mathrm{~h}$ ); column chromatography (petroleum ether/EtOAc $3: 1$ ). Colorless oil. ${ }^{1} \mathrm{H}$ NMR (400 MHz, $\left.\mathrm{CDCl}_{3}\right) \delta 2.94(\mathrm{~s}, 2 \mathrm{H}), 3.61(\mathrm{~s}, 3 \mathrm{H}), 3.81(\mathrm{~s}, 3 \mathrm{H}), 3.89$ (s, 3H), 6.80 (d, J = 8.5 $\mathrm{Hz}, 1 \mathrm{H}), 6.99(\mathrm{~d}, J=1.7 \mathrm{~Hz}, 1 \mathrm{H}), 7.09(\mathrm{dd}, J=8.5$ and $1.7 \mathrm{~Hz}, 1 \mathrm{H}), 7.69(\mathrm{~d}, J=8.7 \mathrm{~Hz}, 2 \mathrm{H})$, $8.23(\mathrm{~d}, J=8.7 \mathrm{~Hz}, 2 \mathrm{H}) ;{ }^{13} \mathrm{C}$ NMR $\left(100 \mathrm{MHz}, \mathrm{CDCl}_{3}\right) \delta 53.9,55.6,55.9,94.3,111.1,112.0$, 121.5, 123.2, 123.7, 125.5, 130.7, 137.0, 147.7, 148.8, 151.4, 156.9, 166.7, 169.4; HRMS (ESI) $\mathrm{m} / \mathrm{z}$ : [M-H] ${ }^{-}$Calcd for $\mathrm{C}_{20} \mathrm{H}_{17} \mathrm{~N}_{2} \mathrm{O}_{8}{ }^{-} 413.0990$; Found 413.0982 . 
Methyl 2-amino-3-(2,3-dihydrobenzo[b][1,4]dioxin-6-yl)-4-(4-nitrophenyl)-5-oxo-2,5dihydrofuran-2-carboxylate (4i)

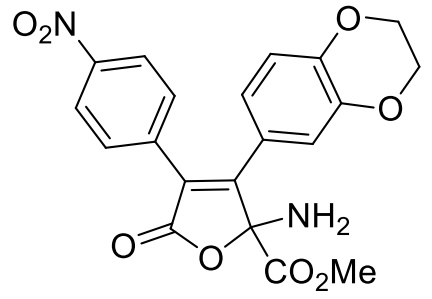

Compound 4i was obtained from azirine $1 \mathbf{i}$ and acid $2 \mathbf{a}$ according to method A (70 mg, 72\%, reaction time $48 \mathrm{~h}$ ); column chromatography (petroleum ether/EtOAc $3: 1$ ). Colorless solid; mp $128-129{ }^{\circ} \mathrm{C}\left(\mathrm{Et}_{2} \mathrm{O} /\right.$ hexane $^{1}{ }^{1} \mathrm{H}$ NMR $\left(400 \mathrm{MHz}, \mathrm{CDCl}_{3}\right) \delta 2.89(\mathrm{~s}, 2 \mathrm{H}), 3.84(\mathrm{~s}, 3 \mathrm{H}), 4.23-4.30$ $(\mathrm{m}, 4 \mathrm{H}), 6.79(\mathrm{~d}, J=8.5 \mathrm{~Hz}, 1 \mathrm{H}), 6.87(\mathrm{dd}, J=8.5$ and $2.1 \mathrm{~Hz}, 1 \mathrm{H}), 6.99(\mathrm{~d}, J=2.1 \mathrm{~Hz}, 1 \mathrm{H})$, $7.68(\mathrm{~d}, J=8.8 \mathrm{~Hz}, 2 \mathrm{H}), 8.23(\mathrm{~d}, J=8.8 \mathrm{~Hz}, 2 \mathrm{H}) ;{ }^{13} \mathrm{C} \mathrm{NMR}\left(100 \mathrm{MHz}, \mathrm{CDCl}_{3}\right) \delta 54.0,64.1$, 64.5, 94.4, 117.9, 118.2, 122.3, 123.0, 123.7, 126.2, 130.6, 136.7, 143.6, 146.1, 147.8, 156.9, 166.6, 169.4; HRMS (ESI) m/z: [M+Na] ${ }^{+}$Calcd for $\mathrm{C}_{20} \mathrm{H}_{16} \mathrm{~N}_{2} \mathrm{NaO}_{8}{ }^{+} 435.0799$; Found 435.0793.

\section{Methyl 2-amino-3,4-bis(4-nitrophenyl)-5-oxo-2,5-dihydrofuran-2-carboxylate $(4 \mathbf{j})$}

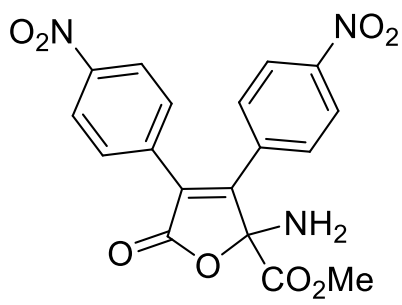

Compound $\mathbf{4} \mathbf{j}$ was obtained from azirine $\mathbf{1} \mathbf{j}$ and acid $\mathbf{2 a}$ according to method A (93 $\mathrm{mg}, 98 \%$, reaction time $48 \mathrm{~h}$ ); column chromatography (petroleum ether/EtOAc $3: 1$ ). Colorless solid; mp 169-170 ${ }^{\circ} \mathrm{C}\left(\mathrm{Et}_{2} \mathrm{O} / \mathrm{hexane}\right){ }^{1} \mathrm{H}$ NMR $\left(400 \mathrm{MHz}, \mathrm{CDCl}_{3}-\mathrm{DMSO}_{6}-\mathrm{d}_{6}\right.$ mixture) $\delta 3.77$ (s, 3H), 4.03 $(\mathrm{s}, 2 \mathrm{H}), 7.59-7.67(\mathrm{~m}, 4 \mathrm{H}), 8.20(\mathrm{~m}, 4 \mathrm{H}) ;{ }^{13} \mathrm{C} \mathrm{NMR}\left(100 \mathrm{MHz}, \mathrm{CDCl}_{3}-\mathrm{DMSO}_{6} \mathrm{~d}_{6}\right.$ mixture $) \delta$ 53.9, 96.8, 123.9 (2C), 130.1, 130.7, 131.0, 135.9, 137.2, 148.0, 148.3, 155.9, 166.2, 169.1; HRMS (ESI) m/z: [M-H] Calcd for $\mathrm{C}_{18} \mathrm{H}_{12} \mathrm{~N}_{3} \mathrm{O}_{8}{ }^{-}$398.0630; Found 398.0620.

Methyl 2-amino-3-(4-cyanophenyl)-4-(4-nitrophenyl)-5-oxo-2,5-dihydrofuran-2carboxylate (4k)

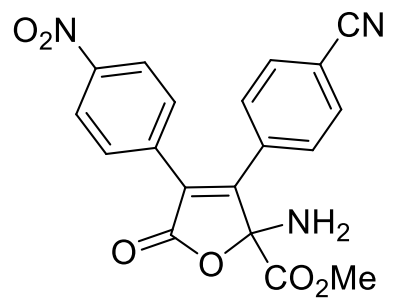

Compound $4 \mathbf{k}$ was obtained from azirine $1 \mathbf{k}$ and acid $\mathbf{2 a}$ according to method A (70 $\mathrm{mg}, 78 \%$, reaction time $48 \mathrm{~h}$ ); column chromatography (petroleum ether/EtOAc $3: 1$ ). Colorless solid; mp 
$72-74{ }^{\circ} \mathrm{C}\left(\mathrm{Et}_{2} \mathrm{O} / \mathrm{hexane}\right) .{ }^{1} \mathrm{H} \mathrm{NMR}\left(400 \mathrm{MHz}, \mathrm{CDCl}_{3}\right) \delta 3.01(\mathrm{~s}, 2 \mathrm{H}), 3.82(\mathrm{~s}, 3 \mathrm{H}), 7.54-7.64(\mathrm{~m}$, $6 \mathrm{H}), 8.17(\mathrm{~d}, J=8.8 \mathrm{~Hz}, 2 \mathrm{H}) ;{ }^{13} \mathrm{C} \mathrm{NMR}\left(100 \mathrm{MHz}, \mathrm{CDCl}_{3}\right) \delta 54.1,94.6,114.1,117.7,123.8$, 129.7, 129.9, 130.5, 132.5, 134.1, 135.0, 148.1, 155.1, 165.6, 168.5; HRMS (ESI) m/z: [M-H] Calcd for $\mathrm{C}_{19} \mathrm{H}_{12} \mathrm{~N}_{3} \mathrm{O}_{6}{ }^{-} 378.0732$; Found 378.0739.

Methyl 2-amino-3-methyl-4-(4-nitrophenyl)-5-oxo-2,5-dihydrofuran-2-carboxylate (4l)

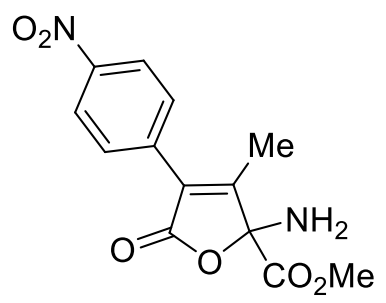

Compound $4 \mathbf{l}$ was obtained from azirine $\mathbf{1 l}$ and acid 2a according to method A (66 mg, 95\%, reaction time $24 \mathrm{~h}$ ); column chromatography (petroleum ether/EtOAc $3: 1$ ). Colorless solid; mp 152-153 ${ }^{\circ} \mathrm{C}\left(\mathrm{Et}_{2} \mathrm{O} / \mathrm{hexane}\right) .{ }^{1} \mathrm{H}$ NMR $\left(400 \mathrm{MHz}, \mathrm{CDCl}_{3}\right) \delta 2.23(\mathrm{~s}, 3 \mathrm{H}), 2.76(\mathrm{~s}, 2 \mathrm{H}), 3.86(\mathrm{~s}$, $3 \mathrm{H}), 7.73(\mathrm{~d}, J=8.8 \mathrm{~Hz}, 2 \mathrm{H}), 8.30(\mathrm{~d}, J=8.8 \mathrm{~Hz}, 2 \mathrm{H}) ;{ }^{13} \mathrm{C} \mathrm{NMR}\left(100 \mathrm{MHz}, \mathrm{CDCl}_{3}\right) \delta 13.0$, 53.8, 95.0, 123.6, 128.0, 130.0, 135.7, 147.8, 159.2, 166.0, 169.2; HRMS (ESI) m/z: [M-H] Calcd for $\mathrm{C}_{13} \mathrm{H}_{11} \mathrm{~N}_{2} \mathrm{O}_{6}{ }^{-} 291.0623$; Found 291.0611.

Methyl 2-amino-5-oxo-3,4-diphenyl-2,5-dihydrofuran-2-carboxylate (4m)<smiles>CC(=O)C1(N)OC(=O)C(c2ccccc2)=C1c1ccccc1</smiles>

Compound $\mathbf{4 m}$ was obtained from azirine $\mathbf{1 a}$ and acid $\mathbf{2 b}$ according to method A (70 $\mathrm{mg}, 95 \%$, reaction time $72 \mathrm{~h}$ ); column chromatography (petroleum ether/EtOAc $3: 1$ ). Colorless solid; mp $123-124{ }^{\circ} \mathrm{C}\left(\mathrm{Et}_{2} \mathrm{O} / \mathrm{hexane}\right) .{ }^{1} \mathrm{H}$ NMR $\left(400 \mathrm{MHz}, \mathrm{CDCl}_{3}\right) \delta 2.84(\mathrm{~s}, 2 \mathrm{H}), 3.82(\mathrm{~s}, 3 \mathrm{H}), 7.28-7.47$ $(\mathrm{m}, 10 \mathrm{H}) ;{ }^{13} \mathrm{C} \mathrm{NMR}\left(100 \mathrm{MHz}, \mathrm{CDCl}_{3}\right) \delta 53.7,94.2,128.5,128.6,129.0,129.1,129.4,129.5$, 129.7, 130.1, 130.3, 154.7, 167.0, 170.2; HRMS (ESI) m/z: $[\mathrm{M}+\mathrm{Na}]^{+}$Calcd for $\mathrm{C}_{18} \mathrm{H}_{15} \mathrm{NNaO}_{4}{ }^{+}$ 332.0893; Found 332.0898.

Methyl 2-amino-4-(4-fluorophenyl)-5-oxo-3-phenyl-2,5-dihydrofuran-2-carboxylate (4n)<smiles>CC(=O)C1(N)OC(=O)C(c2ccc(F)cc2)=C1c1ccccc1</smiles>

Compound 4n was obtained from azirine 1a and acid 2c according to method A (65 mg, 84\%, reaction time $60 \mathrm{~h}$ ); column chromatography (petroleum ether/EtOAc $3: 1$ ). Colorless solid; mp 
153-154 ${ }^{\circ} \mathrm{C}\left(\mathrm{Et}_{2} \mathrm{O} /\right.$ hexane). ${ }^{1} \mathrm{H}$ NMR $\left(400 \mathrm{MHz}, \mathrm{CDCl}_{3}\right) \delta 2.85(\mathrm{~s}, 2 \mathrm{H}), 3.82(\mathrm{~s}, 3 \mathrm{H}), 7.01-7.06$ $(\mathrm{m}, 2 \mathrm{H}), 7.31-7.47(\mathrm{~m}, 7 \mathrm{H}) ;{ }^{13} \mathrm{C} \mathrm{NMR}\left(100 \mathrm{MHz}, \mathrm{CDCl}_{3}\right) \delta 53.8,94.3,115.6(\mathrm{~d}, J=21.6 \mathrm{~Hz})$, 125.5 (d, $J=3.5 \mathrm{~Hz}), 128.7$ (2C), 128.9 (2C), 130.2, 131.5 (d, $J=8.4 \mathrm{~Hz}), 154.8,163.0$ (d, $J=$ $250.1 \mathrm{~Hz}$ ), 166.9, 170.1; HRMS (ESI) m/z: $[\mathrm{M}+\mathrm{H}]^{+}$Calcd for $\mathrm{C}_{18} \mathrm{H}_{15} \mathrm{FNO}_{4}{ }^{+}$328.0980; Found 328.0980 .

Methyl 2-amino-4-(4-chlorophenyl)-5-oxo-3-phenyl-2,5-dihydrofuran-2-carboxylate (40) (CCDC 1902292)

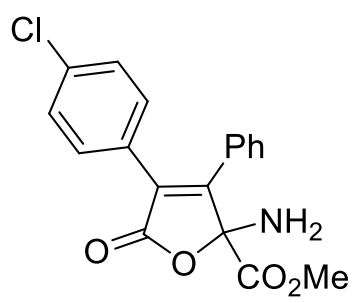

Compound 4o was obtained from azirine 1a and acid 2d according to method A (57 mg, 70\%, reaction time $60 \mathrm{~h}$ ); column chromatography (petroleum ether/EtOAc $3: 1$ ). Colorless solid; mp 161-162 ${ }^{\circ} \mathrm{C}\left(\mathrm{Et}_{2} \mathrm{O} /\right.$ hexane). ${ }^{1} \mathrm{H}$ NMR $\left(400 \mathrm{MHz}, \mathrm{CDCl}_{3}\right) \delta 2.85(\mathrm{~s}, 2 \mathrm{H}), 3.83(\mathrm{~s}, 3 \mathrm{H}), 7.31-7.42$ $(\mathrm{m}, 9 \mathrm{H}) ;{ }^{13} \mathrm{C} \mathrm{NMR}\left(100 \mathrm{MHz}, \mathrm{CDCl}_{3}\right) \delta 53.8,94.3,127.9,128.6,128.8(2 \mathrm{C}), 128.9,130.0$, 130.3, 130.8, 135.3, 155.2, 166.8, 169.9; HRMS (ESI) m/z: $[\mathrm{M}+\mathrm{H}]^{+}$Calcd for $\mathrm{C}_{18} \mathrm{H}_{15}{ }^{35} \mathrm{ClNO}_{4}{ }^{+}$ 344.0684; Found 344.0687.

Methyl 2-amino-4-(2-bromophenyl)-5-oxo-3-phenyl-2,5-dihydrofuran-2-carboxylate (4p)<smiles>CC(=O)C1(N)OC(=O)C(c2ccccc2Br)=C1c1ccccc1</smiles>

Compound 4p was obtained from azirine 1a and acid $\mathbf{2 e}$ according to method A (85 mg, 93\%, reaction time $72 \mathrm{~h}$ ); column chromatography (petroleum ether/EtOAc $3: 1$ ). Colorless solid; mp $133-134{ }^{\circ} \mathrm{C}\left(\mathrm{Et}_{2} \mathrm{O} /\right.$ hexane $) .1$ : 1.2 mixture of rotamers. ${ }^{1} \mathrm{H}$ NMR $\left(400 \mathrm{MHz}, \mathrm{CDCl}_{3}\right) \delta 2.95(\mathrm{~s}$, 4.4), 3.80 (s, 3H), 3.84 (s, 3.6H), 7.25-7.44 (m, 17.6 H), 7.67-7.71 (m, 2.2H); ${ }^{13} \mathrm{C}$ NMR (100 $\left.\mathrm{MHz}, \mathrm{CDCl}_{3}\right) \delta 53.7,53.9,94.5$ (2C), 123.4, 123.6, 127.8, 127.9, 128.5 (2C), 128.8, 128.9, 129.5, 129.8 (2C), 130.2, 130.5 (2C), 130.6 (2C), 130.9, 131.3, 131.7, 132.0, 133.1, 133.4, 155.8, 155.9, 167.0 (2C), 169.2, 169.4; HRMS (ESI) m/z: $[\mathrm{M}+\mathrm{H}]^{+}$Calcd for $\mathrm{C}_{18} \mathrm{H}_{15}{ }^{79} \mathrm{BrNO}_{4}{ }^{+}$ 388.0179; Found 388.0176. 


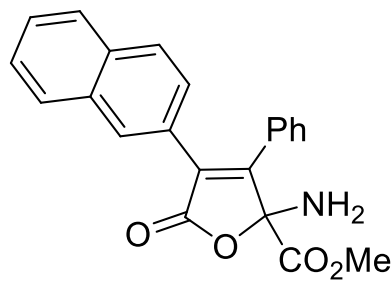

Compound $\mathbf{4 q}$ was obtained from azirine $1 \mathbf{a}$ and acid $\mathbf{2 f}$ according to method A (77 $\mathrm{mg}, 90 \%$ reaction time $72 \mathrm{~h}$ ); column chromatography (petroleum ether/EtOAc $3: 1$ ). Colorless solid; mp 169-170 ${ }^{\circ} \mathrm{C}\left(\mathrm{Et}_{2} \mathrm{O} / \mathrm{hexane}\right) .{ }^{1} \mathrm{H}$ NMR $\left(400 \mathrm{MHz}, \mathrm{CDCl}_{3}\right) \delta 2.90(\mathrm{~s}, 2 \mathrm{H}), 3.85(\mathrm{~s}, 3 \mathrm{H}), 7.28-7.32$ $(\mathrm{m}, 2 \mathrm{H}), 7.36-7.40(\mathrm{~m}, 2 \mathrm{H}), 7.44-7.46(\mathrm{~m}, 2 \mathrm{H}), 7.49-7.56(\mathrm{~m}, 2 \mathrm{H}), 7.75-7.77(\mathrm{~m}, 1 \mathrm{H}), 7.82-$ $7.86(\mathrm{~m}, 2 \mathrm{H}), 8.17(\mathrm{~s}, 1 \mathrm{H}) ;{ }^{13} \mathrm{C}$ NMR $\left(100 \mathrm{MHz}, \mathrm{CDCl}_{3}\right) \delta 53.8,94.2,126.3,126.4,126.9,127.0$, 127.6, 128.0, 128.5, 128.6, 129.1, 129.5, 129.6, 130.2, 130.3, 133.1, 133.3, 154.8, 167.1, 170.3; HRMS (ESI) m/z: [M+H] ${ }^{+}$Calcd for $\mathrm{C}_{22} \mathrm{H}_{18} \mathrm{NO}_{4}{ }^{+} 360.1231$; Found 360.1230.

Methyl 2-amino-5-oxo-3-phenyl-4-(thiophen-2-yl)-2,5-dihydrofuran-2-carboxylate (4r)<smiles>CC(=O)C1(N)OC(=O)C(c2cccs2)=C1c1ccccc1</smiles>

Compound 4r was obtained from azirine 1a and acid $2 \mathrm{~g}$ according to method A $(63 \mathrm{mg}, 85 \%$, reaction time $140 \mathrm{~h}$ ); column chromatography (petroleum ether/EtOAc $3: 1$ ). Colorless oil. ${ }^{1} \mathrm{H}$ NMR (400 MHz, $\left.\mathrm{CDCl}_{3}\right) \delta 2.79(\mathrm{~s}, 2 \mathrm{H}), 3.83(\mathrm{~s}, 3 \mathrm{H}), 6.98-7.01(\mathrm{~m}, 1 \mathrm{H}), 7.37(\mathrm{~d}, J=5.0 \mathrm{~Hz}$, $1 \mathrm{H}), 7.41-7.42(\mathrm{~m}, 2 \mathrm{H}), 7.47-7.48(\mathrm{~m}, 3 \mathrm{H}), 7.60(\mathrm{~d}, J=3.5 \mathrm{~Hz}, 1 \mathrm{H}) ;{ }^{13} \mathrm{C}$ NMR $(100 \mathrm{MHz}$, $\left.\mathrm{CDCl}_{3}\right) \delta 53.7,94.8,124.4,127.0,128.5,128.6,129.0,129.7,130.0,130.4,130.7,151.8,166.6$, 169.3; HRMS (ESI) m/z: [M+Na $]^{+}$Calcd for $\mathrm{C}_{16} \mathrm{H}_{13} \mathrm{NNaO}_{4} \mathrm{~S}^{+} 338.0457$; Found 338.0461.

\section{Dimethyl 2-amino-5-oxo-3-phenyl-2,5-dihydrofuran-2,4-dicarboxylate (4s)}<smiles>COC(=O)C1=C(c2ccccc2)C(N)(C(C)=O)OC1=O</smiles>

Compound 4s was obtained from azirine 1a and acid $\mathbf{2 h}$ according to method A (19 $\mathrm{mg}, 28 \%$, reaction time $48 \mathrm{~h}$ ); column chromatography (petroleum ether/EtOAc $3: 1$ ). Compound $4 \mathrm{~s}$ was also obtained according to method $\mathrm{B}$ from azirine $3 \mathrm{~b}$ and $\mathrm{Et}_{3} \mathrm{~N}$ (45 mg, 64\% on two steps, reaction time $48 \mathrm{~h}$ ). Colorless solid; mp $98-100{ }^{\circ} \mathrm{C}\left(\mathrm{Et}_{2} \mathrm{O} / \mathrm{hexane}\right) .{ }^{1} \mathrm{H} \mathrm{NMR}\left(400 \mathrm{MHz}, \mathrm{CDCl}_{3}\right)$ $\delta 2.89(\mathrm{~s}, 2 \mathrm{H}), 3.80(\mathrm{~s}, 3 \mathrm{H}), 3.88(\mathrm{~s}, 3 \mathrm{H}), 7.43-7.51(\mathrm{~m}, 3 \mathrm{H}), 7.59-7.61(\mathrm{~m}, 2 \mathrm{H}) ;{ }^{13} \mathrm{C} \mathrm{NMR}(100$ $\left.\mathrm{MHz}_{1} \mathrm{CDCl}_{3}\right) \delta 52.9,54.0,94.6,122.8,128.6,128.8,128.9,131.7,161.9,162.4,166.0,166.2$; HRMS (ESI) m/z: [M+Na] ${ }^{+}$Calcd for $\mathrm{C}_{14} \mathrm{H}_{13} \mathrm{NNaO}_{6}{ }^{+} 314.0635$; Found 314.0647. 


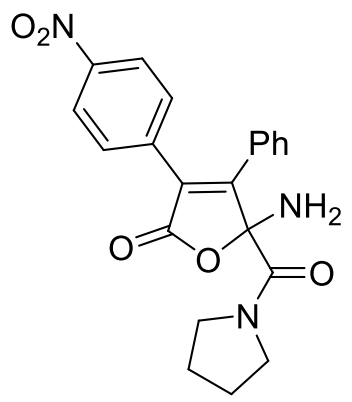

Compounds $\mathbf{4 t}(7 \mathrm{mg}, 8 \%)$ and $\mathbf{5 j}$ (70 $\mathrm{mg}, 74 \%)$ were obtained from azirine $\mathbf{1 m}$ and acid $\mathbf{2 a}$ according to method A (reaction time $48 \mathrm{~h}$ ) and separated by column chromatography (petroleum ether/EtOAc $2: 1$ ). Compounds $4 \mathbf{t}$ (56 mg, 59\% on two steps) and $\mathbf{5 j}$ (37 mg, 39\% on two steps) were also obtained according to method $\mathrm{B}$ from azirine $3 \mathbf{c}$ and $\mathrm{Et}_{3} \mathrm{~N}$ (reaction time 48 h). Compound 4t: colorless solid; mp 184-185 ${ }^{\circ} \mathrm{C}\left(\mathrm{Et}_{2} \mathrm{O} /\right.$ hexane). ${ }^{1} \mathrm{H}$ NMR $\left(400 \mathrm{MHz}, \mathrm{CDCl}_{3}\right) \delta$ 1.77-2.00 (m, 4H), 3.10 (br.s, 2H), 3.39-3.67 (m, 4H), 7.34-7.47 (m, 5H), 7.66 (d, J = 8.8 Hz, $2 \mathrm{H}), 8.23(\mathrm{~d}, J=8.8 \mathrm{~Hz}, 2 \mathrm{H}) ;{ }^{13} \mathrm{C} \mathrm{NMR}\left(100 \mathrm{MHz} \mathrm{CDCl}_{3}\right) \delta$ 23.1, 27.0, 46.3, 49.1, 95.6, 123.9, 126.5, 128.8, 129.3, 129.4, 130.4, 131.3, 136.1, 148.0, 159.3, 162.9, 170.0; HRMS (ESI) m/z: $[\mathrm{M}-\mathrm{H}]^{-}$Calcd for $\mathrm{C}_{21} \mathrm{H}_{18} \mathrm{~N}_{3} \mathrm{O}_{5}{ }^{-} 392.1252$; Found 392.1243.

\section{5-Amino-3-(4-chlorophenyl)-4-phenyl-5-(pyrrolidine-1-carbonyl)furan-2(5H)-one (4u)}

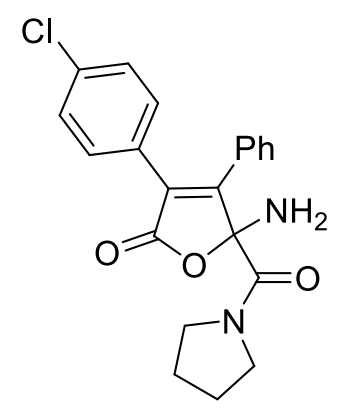

Compounds 4u (62 mg, 68\%) and 5k (12 mg, 13\%) were obtained from azirine $\mathbf{1 m}$ and acid $\mathbf{2 d}$ according to method A (reaction time $140 \mathrm{~h}$ ) and separated by column chromatography (petroleum ether/EtOAc 2 : 1). Compound 4u: colorless solid; mp $175-177{ }^{\circ} \mathrm{C}\left(\mathrm{Et}_{2} \mathrm{O} /\right.$ hexane $) .{ }^{1} \mathrm{H}$ NMR (400 MHz, $\left.\mathrm{CDCl}_{3}\right) \delta$ 1.74-1.99 (m, 4H), $3.04(\mathrm{~s}, 2 \mathrm{H}), 3.35-3.67$ (m, 4H), 7.31-7.43 (m, $9 \mathrm{H}) ;{ }^{13} \mathrm{C} \mathrm{NMR}\left(100 \mathrm{MHz}, \mathrm{CDCl}_{3}\right) \delta 23.1,26.9,46.2,48.9,95.3,127.4,127.9,128.6,129.0(2 \mathrm{C})$, $129.9,130.6,130.7,135.3,156.7,163.2,170.7$; HRMS (ESI) m/z: $[\mathrm{M}+\mathrm{Na}]^{+} \mathrm{Calcd}$ for $\mathrm{C}_{21} \mathrm{H}_{19}{ }^{35} \mathrm{ClN}_{2} \mathrm{NaO}_{3}{ }^{+} 405.0976$; Found 405.0969. 
2-Amino- $N$-benzyl- $N$-methyl-4-(4-nitrophenyl)-5-oxo-3-phenyl-2,5-dihydrofuran-2carboxamide (4w) and 2-amino- $N$-benzyl- $N$-methyl-4-(4-nitrophenyl)-5-oxo-2-phenyl-2,5dihydrofuran-3-carboxamide (5n)

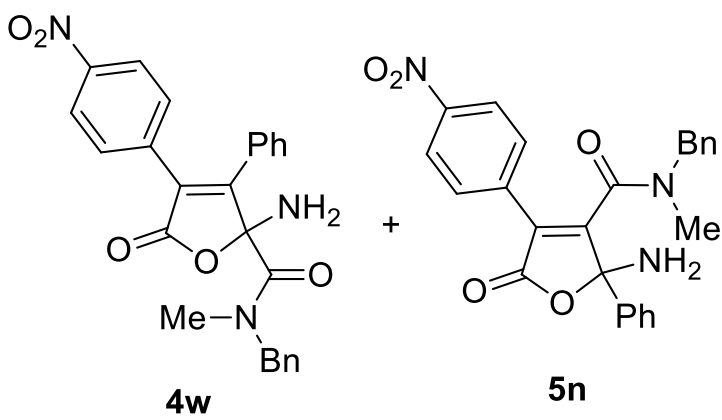

Unseparated 1 : 3.4 mixture of $\mathbf{4 w}$ and $\mathbf{5 n}(83 \mathrm{mg}, 79 \%)$ was obtained from azirine $\mathbf{1 n}$ and acid 2a according to method A (reaction time $48 \mathrm{~h}$ ); column chromatography (petroleum ether/EtOAc 2 : 1). Colorless solid. ${ }^{1} \mathrm{H}$ NMR $\left(400 \mathrm{MHz}, \mathrm{CDCl}_{3}\right) \delta 1.78(\mathrm{~s}, 8.6 \mathrm{H}), 2.77(\mathrm{~s}, 3 \mathrm{H}), 3.02(\mathrm{~s}, 9.9 \mathrm{H})$, $4.52-4.53(\mathrm{~m}, 6.6 \mathrm{H}), 6.13(\mathrm{~s}, 1 \mathrm{H}), 6.92(\mathrm{~s}, 1 \mathrm{H}), 7.20-7.21(\mathrm{~m}, 7 \mathrm{H}), 7.31-7.36(\mathrm{~m}, 24.4 \mathrm{H}), 7.46-$ 7.47 (m, 3H), 7.61-7.63 (m, 6.6H), 7.70 (br.m, 2H), 7.81-7.86 (m, 8.6H), 8.09-8.11 (m, 6.6H), 8.21-8.23 (m, 2H); ${ }^{13} \mathrm{C}$ NMR $\left(100 \mathrm{MHz}, \mathrm{CDCl}_{3}\right) \delta 32.0,33.8,50.2,53.0,97.7,97.9,123.7(2 \mathrm{C})$, $124.1,125.2,126.0,126.2,126.6,127.7,128.2$, 128.4, 128.7, 128.9, 129.0, 129.1, 129.2, 129.7 (2C), 129.9, 133.8, 134.3, 134.7, 134.9, 137.2, 137.4, 148.2, 148.3, 156.6, 156.9, 162.8, 163.1, 168.6, 168.7; HRMS (ESI) m/z: [M-H] Calcd for $\mathrm{C}_{25} \mathrm{H}_{20} \mathrm{~N}_{3} \mathrm{O}_{5}{ }^{-} 442.1408$; Found 442.1397.

5-Amino-5-(morpholine-4-carbonyl)-3-(4-nitrophenyl)-4-phenylfuran-2(5H)-one (4x) and 5-amino-4-(morpholine-4-carbonyl)-3-(4-nitrophenyl)-5-phenylfuran-2(5H)-one (5o)

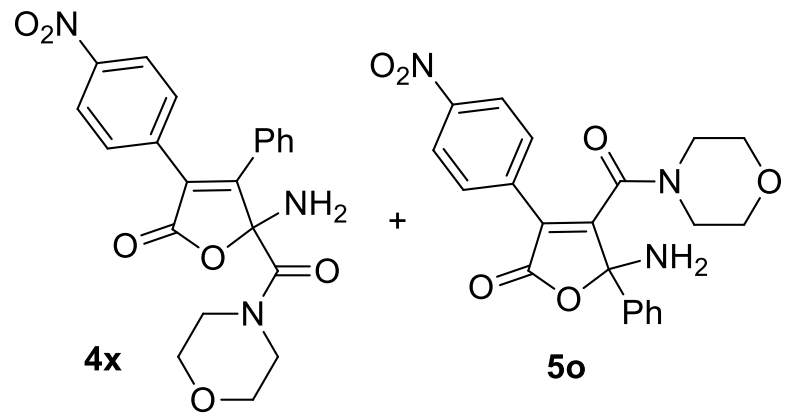

Unseparated $1: 2.5$ mixture of $\mathbf{4 x}$ and $\mathbf{5 0}$ (91 $\mathrm{mg}$, 93\%) was obtained from azirine 10 and acid 2a according to method A (reaction time $48 \mathrm{~h}$ ); column chromatography (petroleum ether/EtOAc 2 : 1). Colorless solid. ${ }^{1} \mathrm{H}$ NMR (400 MHz, $\mathrm{CDCl}_{3}$ ) $\delta 2.20$ (br.m, 2H), 2.41 (br.m, 5H), 2.69 (br.m, 3H), 3.01 (br.m, 6H), 3.41-3.52 (m, 8H), 3.66-3.69 (m, 11H), 7.35-7.39 (m, 2H), 7.45-7.52 (m, $10.5 \mathrm{H}), 7.63-7.67(\mathrm{~m}, 7 \mathrm{H}), 7.91(\mathrm{~d}, J=8.7 \mathrm{~Hz}, 5 \mathrm{H}), 8.19(\mathrm{~d}, J=8.7 \mathrm{~Hz}, 2 \mathrm{H}), 8.28(\mathrm{~d}, J=8.7 \mathrm{~Hz}$ $5 \mathrm{H}) ;{ }^{13} \mathrm{C} \mathrm{NMR}\left(100 \mathrm{MHz}, \mathrm{CDCl}_{3}\right) \delta 41.5,45.6(2 \mathrm{C}), 45.9,65.4,65.9,66.5(2 \mathrm{C}), 95.8,97.7$, $123.7,124.7,123.9,124.7,126.1$ (2C), 129.1, 129.2, 129.3 (2C), 130.0, 130.5, 131.1, 134.2, 136.0, 137.0, 147.9, 148.4, 155.6, 161.2, 161.4, 163.8, 168.5, 169.3; HRMS (ESI) m/z: [M-H] Calcd for $\mathrm{C}_{21} \mathrm{H}_{18} \mathrm{~N}_{3} \mathrm{O}_{6}{ }^{-} 408.1201$; Found 408.1194 . 
tert-Butyl 2-amino-4-(4-nitrophenyl)-5-oxo-3-phenyl-2,5-dihydrofuran-2-carboxylate (4y) and tert-butyl 2-amino-4-(4-nitrophenyl)-5-oxo-2-phenyl-2,5-dihydrofuran-3-carboxylate (5p)

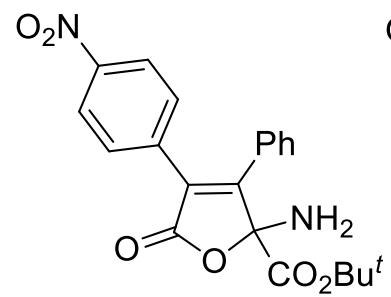

$4 y$<smiles>CCCCOC(=O)C1=C(c2ccc([N+](=O)[O-])cc2)C(=O)OC1(N)c1ccccc1</smiles>

$5 p$

Unseparated 1: 1 mixture of $\mathbf{4 y}$ and $\mathbf{5 p}(76 \mathrm{mg}, 80 \%)$ was obtained from azirine $1 \mathbf{p}$ and acid 2a according to method A (reaction time $24 \mathrm{~h}$ ); column chromatography (petroleum ether/EtOAc 3 : 1). Unseparated 1 : 5 mixture of $\mathbf{4 y}$ and $\mathbf{5 p}(80 \mathrm{mg}, 85 \%)$ was obtained from azirine $\mathbf{1 p}$ and acid 2a using $\mathrm{Et}_{3} \mathrm{~N}$ as a base (reaction time $\left.24 \mathrm{~h}\right)$. Compound $4 \mathbf{y}:{ }^{1} \mathrm{H} \mathrm{NMR}\left(400 \mathrm{MHz}, \mathrm{CDCl}_{3}\right) \delta 1.41$ (s, 9H), $2.87(\mathrm{~s}, 2 \mathrm{H}), 7.32-7.36(\mathrm{~m}, 2 \mathrm{H}), 7.40-7.43(\mathrm{~m}, 3 \mathrm{H}), 7.61-7.64(\mathrm{~m}, 2 \mathrm{H})$ 8.17-8.19 (m, $2 \mathrm{H}) ;{ }^{13} \mathrm{C}$ NMR $\left(100 \mathrm{MHz}, \mathrm{CDCl}_{3}\right) \delta 27.4,84.6,95.2,123.7,127.4,128.8,129.1,129.7,130.4$, 130.7, 136.5, 147.8, 158.2, 164.5, 169.5; HRMS (ESI) m/z: [M-H] Calcd for $\mathrm{C}_{21} \mathrm{H}_{19} \mathrm{~N}_{2} \mathrm{O}_{6}{ }^{-}$ 395.1249; Found 395.1234. Compound 5p: ${ }^{1} \mathrm{H} \mathrm{NMR}\left(400 \mathrm{MHz}, \mathrm{CDCl}_{3}\right) \delta 1.33(\mathrm{~s}, 9 \mathrm{H}), 2.82$ (br.s, 2H), 7.40-7.43 (m, 3H), 7.58-7.61 (m, 2H), 7.82-7.84 (m, 2H), 8.24-8.27 (m, 2H); ${ }^{13} \mathrm{C}$ NMR $\left(100 \mathrm{MHz}, \mathrm{CDCl}_{3}\right) \delta 27.6,84.7,97.6,123.3,126.2,128.3,128.5,129.5,129.9,134.6$, 136.4, 148.3, 154.4, 161.2, 169.0; HRMS (ESI) m/z: [M-H] ${ }^{-}$Calcd for $\mathrm{C}_{21} \mathrm{H}_{19} \mathrm{~N}_{2} \mathrm{O}_{6}{ }^{-} 395.1249$; Found 395.1234. 


\section{General procedure for the synthesis of 2-amino-5-oxo-2,5-dihydrofuran-3-}

carboxylates 5a-m
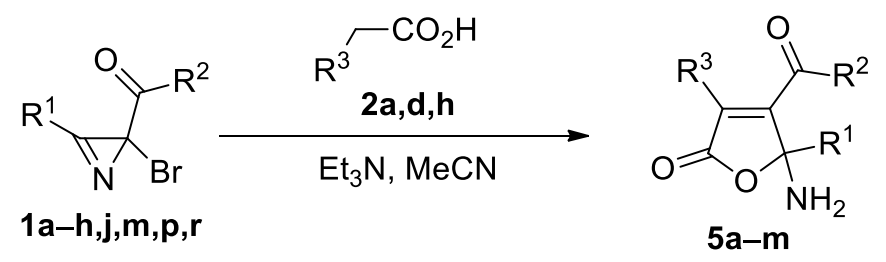

$\mathrm{Et}_{3} \mathrm{~N}(0.47 \mathrm{mmol})$ was added to a solution of 2-bromo- $2 \mathrm{H}$-azirine $1(0.24 \mathrm{mmol})$ and acid $2(0.26$ $\mathrm{mmol}$ ) in $\mathrm{MeCN}(4 \mathrm{~mL})$ at $\mathrm{rt}$, and the reaction mixture was stirred for 24 to $140 \mathrm{~h}$ as indicated below. The solvent was removed in vacuo and the product $\mathbf{5 a - m}$ was purified by a silica gel column chromatography followed by recrystallization.

Methyl 2-amino-4-(4-nitrophenyl)-5-oxo-2-phenyl-2,5-dihydrofuran-3-carboxylate (CCDC 1986590)

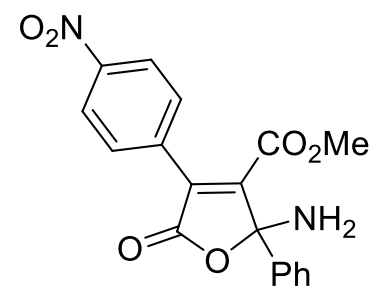

Compounds 4a (10 mg, 12\%) and 5a (60 mg, 71\%) were obtained according to the general procedure from azirine 1a and acid 2a (reaction time $24 \mathrm{~h}$ ) and separated by column chromatography (petroleum ether/EtOAc $3: 1$ ). Compound 5a was also obtained according in the $1 \mathrm{mmol}$ scale reaction $(216 \mathrm{mg}, 61 \%$ on two steps). Compound 5a: colorless solid; mp 162$163{ }^{\circ} \mathrm{C}\left(\mathrm{Et}_{2} \mathrm{O} /\right.$ hexane). ${ }^{1} \mathrm{H}$ NMR $\left(400 \mathrm{MHz}, \mathrm{CDCl}_{3}\right) \delta 2.76$ (s, 2H), 3.73 (s, 3H), 7.44-7.45 (m, $3 \mathrm{H}), 7.59-7.62(\mathrm{~m}, 2 \mathrm{H}), 7.78(\mathrm{~d}, J=8.8 \mathrm{~Hz}, 2 \mathrm{H}), 8.29(\mathrm{~d}, J=8.8 \mathrm{~Hz}, 2 \mathrm{H}) ;{ }^{13} \mathrm{C}$ NMR $(100 \mathrm{MHz}$, $\left.\mathrm{CDCl}_{3}\right) \delta 52.9,97.7,123.5,126.2,128.8,129.7,130.1,130.8,134.4,136.3,148.5,152.5,162.4$, 168.6; HRMS (ESI) m/z: $[\mathrm{M}+\mathrm{H}]^{+}$Calcd for $\mathrm{C}_{18} \mathrm{H}_{15} \mathrm{~N}_{2} \mathrm{O}_{6}{ }^{+} 355.0925$; Found 355.0928.

Methyl

2-amino-2-(4-methylphenyl)-4-(4-nitrophenyl)-5-oxo-2,5-dihydrofuran-3carboxylate (5b)

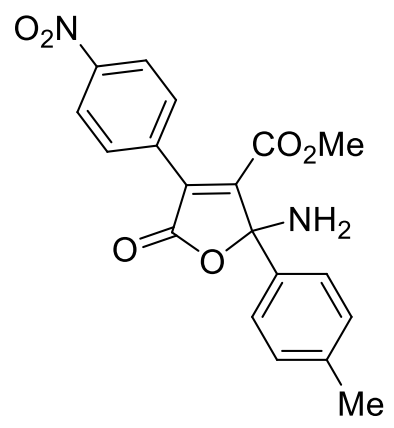

Compound $\mathbf{5 b}$ was obtained according to the general procedure from azirine $\mathbf{1 b}$ and acid $\mathbf{2 a}$ (61 $\mathrm{mg}, 70 \%$, reaction time $24 \mathrm{~h}$ ); column chromatography (petroleum ether/EtOAc $3: 1$ ). Colorless solid; mp $187-188{ }^{\circ} \mathrm{C}\left(\mathrm{Et}_{2} \mathrm{O} /\right.$ hexane). ${ }^{1} \mathrm{H}$ NMR (400 MHz, DMSO-d 6 ) $\delta 2.33$ (s, 3H), 3.59 (s, 
$3 \mathrm{H}), 4.06(\mathrm{~s}, 2 \mathrm{H}), 7.23(\mathrm{~d}, J=8.0 \mathrm{~Hz}, 2 \mathrm{H}), 7.45(\mathrm{~d}, J=8.0 \mathrm{~Hz}, 2 \mathrm{H}), 7.81(\mathrm{~d}, J=8.7 \mathrm{~Hz}, 2 \mathrm{H})$, $8.33(\mathrm{~d}, J=8.7 \mathrm{~Hz}, 2 \mathrm{H}) ;{ }^{13} \mathrm{C}$ NMR $\left(100 \mathrm{MHz}, \mathrm{DMSO}-\mathrm{d}_{6}\right) \delta 21.1,52.9,100.4,123.8,126.9$, 129.3, 130.9, 132.0, 134.6, 135.8, 138.8, 148.3, 152.2, 162.6, 169.3; HRMS (ESI) m/z: [M+H] ${ }^{+}$ Calcd for $\mathrm{C}_{19} \mathrm{H}_{17} \mathrm{~N}_{2} \mathrm{O}_{6}{ }^{+} 369.1081$; Found 369.1070.

\section{Methyl 2-amino-2-(4-(tert-butyl)phenyl)-4-(4-nitrophenyl)-5-oxo-2,5-dihydrofuran-3-} carboxylate (5c)

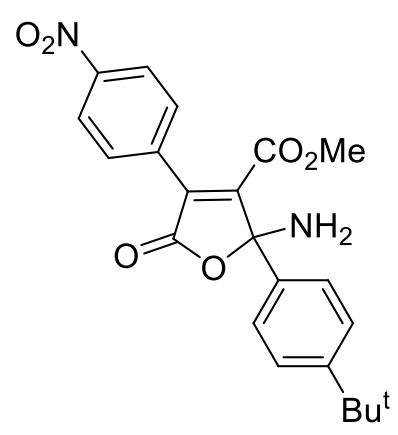

Compound 5c was obtained according to the general procedure from azirine 1c and acid $\mathbf{2 a}$ (73 $\mathrm{mg}, 75 \%$, reaction time $48 \mathrm{~h}$ ); column chromatography (petroleum ether/EtOAc $3: 1$ ). Colorless solid; mp 82-83 ${ }^{\circ} \mathrm{C}\left(\mathrm{Et}_{2} \mathrm{O} /\right.$ hexane). ${ }^{1} \mathrm{H}$ NMR (400 MHz, $\left.\mathrm{CDCl}_{3}\right) \delta 1.35$ (s, 9H), 2.81 (br. s, 2H), $3.74(\mathrm{~s}, 3 \mathrm{H}), 7.45$ and $7.52(\mathrm{AB}-\mathrm{q}, J=8.5 \mathrm{~Hz}, 4 \mathrm{H}), 7.77(\mathrm{~d}, J=8.7 \mathrm{~Hz}, 2 \mathrm{H}), 8.27(\mathrm{~d}, J=8.7 \mathrm{~Hz}$, $2 \mathrm{H}) ;{ }^{13} \mathrm{C}$ NMR $\left(100 \mathrm{MHz}, \mathrm{CDCl}_{3}\right) \delta 31.1,34.6,52.8,97.8,123.4,125.7,125.9,130.1,130.8$, 133.3, 134.5, 148.4, 152.4, 152.9, 162.5, 168.6; HRMS (ESI) m/z: [M-H] Calcd for $\mathrm{C}_{22} \mathrm{H}_{21} \mathrm{~N}_{2} \mathrm{O}_{6}{ }^{-}$ 409.1405; Found 409.1401.

\section{Methyl 2-amino-2-(4-fluorophenyl)-4-(4-nitrophenyl)-5-oxo-2,5-dihydrofuran-3- carboxylate (5d)}

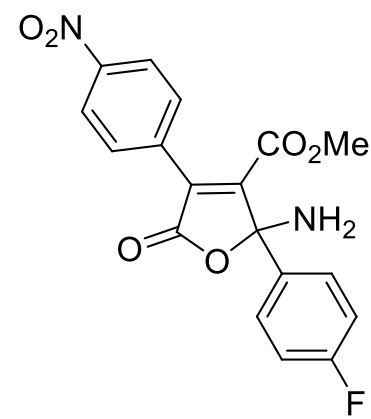

Compound 5d was obtained according to the general procedure from azirine $1 \mathbf{d}$ and acid $\mathbf{2 a}$ (60 $\mathrm{mg}, 68 \%$, reaction time $48 \mathrm{~h}$ ); column chromatography (petroleum ether/EtOAc $3: 1$ ). Colorless solid; mp $167-168{ }^{\circ} \mathrm{C}\left(\mathrm{Et}_{2} \mathrm{O} /\right.$ hexane) ${ }^{1} \mathrm{H}$ NMR (400 MHz, $\mathrm{CDCl}_{3}-\mathrm{DMSO} \mathrm{d}_{6}$ mixture) $\delta 3.59$ (s, $3 \mathrm{H}), 4.01(\mathrm{~s}, 2 \mathrm{H}), 7.14-7.18(\mathrm{~m}, 2 \mathrm{H}), 7.59-7.63(\mathrm{~m}, 2 \mathrm{H}), 7.79(\mathrm{~d}, J=8.8 \mathrm{~Hz}, 2 \mathrm{H}), 8.29(\mathrm{~d}, J=$ $8.8 \mathrm{~Hz}, 2 \mathrm{H}) ;{ }^{13} \mathrm{C}$ NMR $\left(100 \mathrm{MHz}, \mathrm{CDCl}_{3}-\mathrm{DMSO}_{6} \mathrm{~d}_{6}\right.$ mixture) $\delta 52.7,99.5,115.2(\mathrm{~d}, J=21.6$ $\mathrm{Hz}), 123.4,129.3(\mathrm{~d}, J=8.5 \mathrm{~Hz}), 130.8,132.2,133.6$ (d, $J=2.9 \mathrm{~Hz}), 135.6,148.2,151.7,162.3$, $162.8\left(\mathrm{~d}, J=246.4 \mathrm{~Hz}\right.$ ), 169.0; HRMS (ESI) m/z: [M-H] ${ }^{-}$Calcd for $\mathrm{C}_{18} \mathrm{H}_{12} \mathrm{FN}_{2} \mathrm{O}_{6}{ }^{-} 371.0685$; Found 371.0675 . 
Methyl 2-amino-2-(4-chlorophenyl)-4-(4-nitrophenyl)-5-oxo-2,5-dihydrofuran-3carboxylate (5e)

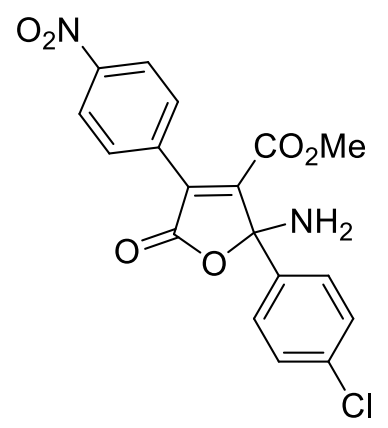

Compound 5e was obtained according to the general procedure from azirine 1e and acid $\mathbf{2 a}$ (63 mg, 69\%, reaction time $48 \mathrm{~h}$ ); column chromatography (petroleum ether/EtOAc $3: 1$ ). Colorless solid; mp 187-189 ${ }^{\circ} \mathrm{C}\left(\mathrm{Et}_{2} \mathrm{O} /\right.$ hexane). ${ }^{1} \mathrm{H}$ NMR (400 MHz, $\mathrm{CDCl}_{3}-\mathrm{DMSO}-\mathrm{d}_{6}$ mixture) $\delta 3.59$ (s, $3 \mathrm{H}), 4.02(\mathrm{~s}, 2 \mathrm{H}), 7.42(\mathrm{~d}, J=8.7 \mathrm{~Hz}, 2 \mathrm{H}), 7.57(\mathrm{~d}, J=8.7 \mathrm{~Hz}, 2 \mathrm{H}), 7.79$ (d, $J=8.9 \mathrm{~Hz}, 2 \mathrm{H})$, $8.29(\mathrm{~d}, J=8.9 \mathrm{~Hz}, 2 \mathrm{H}) ;{ }^{13} \mathrm{C}$ NMR $\left(100 \mathrm{MHz}, \mathrm{CDCl}_{3}-\mathrm{DMSO}_{6} \mathrm{~d}_{6}\right.$ mixture) $\delta 52.7,99.3,123.4$, $128.4,128.9,130.8,132.4,134.3,135.5,136.5,148.2,151.4,162.2,169.0 ;$ HRMS (ESI) m/z: $[\mathrm{M}+\mathrm{Na}]^{+}$Calcd for $\mathrm{C}_{18} \mathrm{H}_{13}{ }^{35} \mathrm{ClN}_{2} \mathrm{NaO}_{6}{ }^{+} 411.0354$; Found 411.0369 .

Methyl

2-amino-2-(4-bromophenyl)-4-(4-nitrophenyl)-5-oxo-2,5-dihydrofuran-3carboxylate (5f)

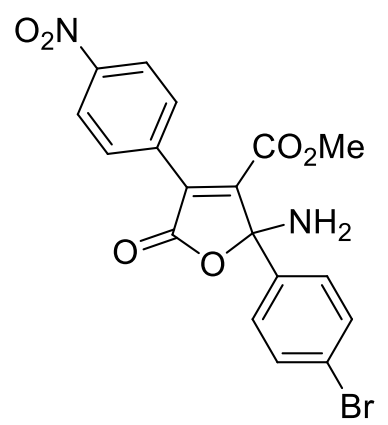

Compounds $\mathbf{5 f}(62 \mathrm{mg}, 61 \%)$ and $\mathbf{4 f}(23 \mathrm{mg}, 23 \%)$ were obtained according to the general procedure from azirine 1f and acid 2a (reaction time $48 \mathrm{~h}$ ) and separated by column chromatography (petroleum ether/EtOAc 3 : 1). Compound 5f: colorless solid; mp $197-198{ }^{\circ} \mathrm{C}$ (Et ${ }_{2} \mathrm{O} /$ hexane). ${ }^{1} \mathrm{H}$ NMR (400 MHz, $\mathrm{CDCl}_{3}-\mathrm{DMSO}-\mathrm{d}_{6}$ mixture) $\delta 3.60(\mathrm{~s}, 3 \mathrm{H}), 4.00(\mathrm{~s}, 2 \mathrm{H}), 7.51$ and $7.56(\mathrm{AB}-\mathrm{q}, J=8.6 \mathrm{~Hz}, 4 \mathrm{H}), 7.79(\mathrm{~d}, J=8.7 \mathrm{~Hz}, 2 \mathrm{H}), 8.29(\mathrm{~d}, J=8.7 \mathrm{~Hz}, 2 \mathrm{H}) ;{ }^{13} \mathrm{C}$ NMR $\left(100 \mathrm{MHz}, \mathrm{CDCl}_{3}-\mathrm{DMSO}_{-} \mathrm{d}_{6}\right.$ mixture) $\delta$ 52.7, 99.3, 122.9, 123.4, 129.2, 130.8, 131.4, 132.4, 135.5, 136.9, 148.2, 151.4, 162.2, 169.0; HRMS (ESI) m/z: [M-H] ${ }^{-}$Calcd for $\mathrm{C}_{18} \mathrm{H}_{12}{ }^{79} \mathrm{BrN}_{2} \mathrm{O}_{6}{ }^{-}$ 430.9884 ; Found 430.9874. 
Methyl 2-amino-2-(4-methoxyphenyl)-4-(4-nitrophenyl)-5-oxo-2,5-dihydrofuran-3carboxylate (5g)

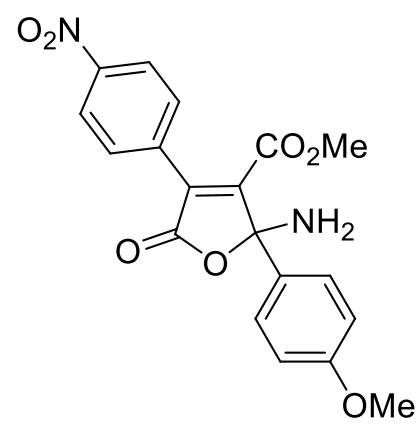

Compound $\mathbf{5 g}$ was obtained according to the general procedure from azirine $\mathbf{1 g}$ and acid $\mathbf{2 a}$ (70 mg, 77\%, reaction time $48 \mathrm{~h}$ ); column chromatography (petroleum ether/EtOAc $3: 1$ ). Colorless solid; mp 166-167 ${ }^{\circ} \mathrm{C}\left(\mathrm{Et}_{2} \mathrm{O} /\right.$ hexane). ${ }^{1} \mathrm{H}$ NMR (400 MHz, $\mathrm{CDCl}_{3}-\mathrm{DMSO}-\mathrm{d}_{6}$ mixture) $\delta 3.60$ (s, $3 \mathrm{H}), 3.78(\mathrm{~s}, 3 \mathrm{H}), 3.84(\mathrm{~s}, 2 \mathrm{H}), 6.90(\mathrm{~d}, J=8.6 \mathrm{~Hz}, 2 \mathrm{H}), 7.46(\mathrm{~d}, J=8.6 \mathrm{~Hz}, 2 \mathrm{H}), 7.77(\mathrm{~d}, J=8.6$ $\mathrm{Hz}, 2 \mathrm{H}), 8.27(\mathrm{~d}, J=8.6 \mathrm{~Hz}, 2 \mathrm{H}) ;{ }^{13} \mathrm{C}$ NMR $\left(100 \mathrm{MHz}, \mathrm{CDCl}_{3}-\mathrm{DMSO}_{6} \mathrm{~d}_{6}\right.$ mixture) $\delta 52.6,55.3$, 99.8, 113.7, 123.4, 128.1, 129.1, 130.6, 131.4, 135.6, 148.1, 152.2, 160.1, 162.4, 169.1; HRMS (ESI) m/z: [M-H] ${ }^{-}$Calcd for $\mathrm{C}_{19} \mathrm{H}_{15} \mathrm{~N}_{2} \mathrm{O}_{7}{ }^{-} 383.0885$; Found 383.0890.

\section{Methyl 2-amino-2-(3,4-dimethoxyphenyl)-4-(4-nitrophenyl)-5-oxo-2,5-dihydrofuran-3-} carboxylate (5h)

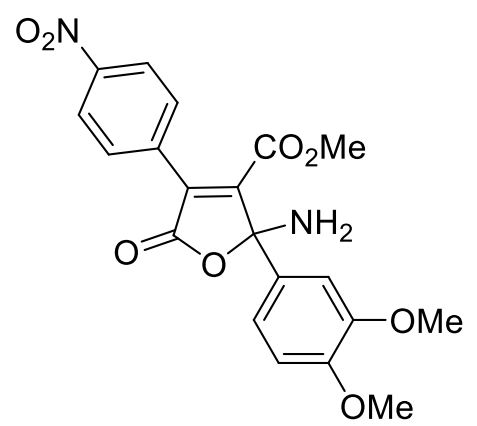

Compound $\mathbf{5 h}$ was obtained according to the general procedure from azirine $\mathbf{1 h}$ and acid $\mathbf{2 a}$ (73 mg, 74\%, reaction time $48 \mathrm{~h}$ ); column chromatography (petroleum ether/EtOAc $3: 1$ ). Colorless oil. ${ }^{1} \mathrm{H}$ NMR (400 MHz, $\mathrm{CDCl}_{3}$ ) $\delta 3.19$ (br. s, 2H), 3.75 (s, 3H), 3.91 (s, 3H), 3.92 (s, 3H), 6.90 $(\mathrm{d}, J=8.5 \mathrm{~Hz}, 1 \mathrm{H}), 7.10(\mathrm{~d}, J=2.0 \mathrm{~Hz}, 1 \mathrm{H}), 7.17(\mathrm{dd}, J=8.5$ and $2.0 \mathrm{~Hz}, 1 \mathrm{H}), 7.78(\mathrm{~d}, J=8.8$ $\mathrm{Hz}, 2 \mathrm{H}), 8.29(\mathrm{~d}, J=8.8 \mathrm{~Hz}, 2 \mathrm{H}) ;{ }^{13} \mathrm{C} \mathrm{NMR}\left(100 \mathrm{MHz}, \mathrm{CDCl}_{3}\right) \delta 52.8,55.8,56.0,97.7,109.5$, $110.9,119.0,123.4,128.4,130.0,130.2$, 134.4, 148.4, 148.9, 150.0, 152.5, 162.5, 168.6; HRMS (ESI) m/z: [M-H] ${ }^{-}$Calcd for $\mathrm{C}_{20} \mathrm{H}_{17} \mathrm{~N}_{2} \mathrm{O}_{8}{ }^{-}$413.0990; Found 413.0983. 


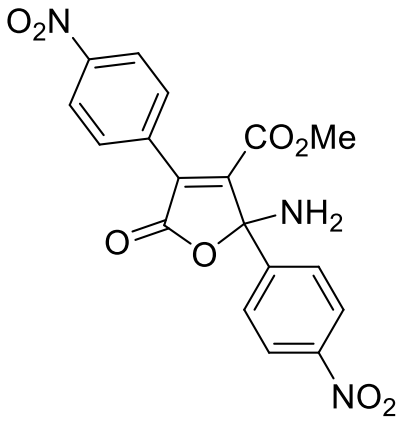

Compounds $\mathbf{5 i}$ (45 $\mathrm{mg}, 48 \%)$ and $\mathbf{4 j}$ (33 $\mathrm{mg}, 35 \%)$ were obtained according to the general procedure from azirine $\mathbf{1 j}$ and acid $\mathbf{2 a}$ (reaction time $48 \mathrm{~h}$ ) and separated by column chromatography (petroleum ether/EtOAc $3: 1$ ). Compound 5i: colorless solid; mp 194-195 ${ }^{\circ} \mathrm{C}$ (Et ${ }_{2} \mathrm{O} /$ hexane). ${ }^{1} \mathrm{H}$ NMR (400 MHz, $\mathrm{CDCl}_{3}-\mathrm{DMSO}-d_{6}$ mixture) $\delta 3.58(\mathrm{~s}, 3 \mathrm{H}), 4.14(\mathrm{~s}, 2 \mathrm{H}), 7.80$ $(\mathrm{d}, J=8.8 \mathrm{~Hz}, 2 \mathrm{H}), 7.86(\mathrm{~d}, J=8.9 \mathrm{~Hz}, 2 \mathrm{H}), 8.23(\mathrm{~d}, J=8.9 \mathrm{~Hz}, 2 \mathrm{H}), 8.30(\mathrm{~d}, J=8.8 \mathrm{~Hz}, 2 \mathrm{H})$; ${ }^{13} \mathrm{C}$ NMR (100 MHz, $\mathrm{CDCl}_{3}-\mathrm{DMSO}_{\mathrm{d}}$ mixture) $\delta$ 52.7, 98.8, 123.4 (2C), 128.7, 130.9, 133.3, 135.3, 144.6, 148.1, 148.3, 150.6, 161.9, 168.8; HRMS (ESI) m/z: [M-H] Calcd for $\mathrm{C}_{18} \mathrm{H}_{12} \mathrm{~N}_{3} \mathrm{O}_{8}{ }^{-}$ 398.0630; Found 398.0626.

\section{5-Amino-3-(4-nitrophenyl)-5-phenyl-4-(pyrrolidine-1-carbonyl)furan-2(5H)-one (5j)}<smiles>NC1(c2ccccc2)OC(=O)C(c2ccc([N+](=O)[O-])cc2)=C1C(=O)N1CCCC1</smiles>

Compound $\mathbf{5 j}$ was obtained according to the general procedure from azirine $\mathbf{1 m}$ and acid $\mathbf{2 a}$ for (76 mg, 82\%, reaction time $48 \mathrm{~h}$ ); column chromatography (petroleum ether/EtOAc $3: 1$ ). Colorless solid; mp $183-184{ }^{\circ} \mathrm{C}\left(\mathrm{Et}_{2} \mathrm{O} /\right.$ hexane). ${ }^{1} \mathrm{H}$ NMR $\left(400 \mathrm{MHz}, \mathrm{CDCl}_{3}\right) \delta 1.16-1.20(\mathrm{~m}$, 1H), 1.28-1.33 (m, 1H), 1.57-1.64 (m, 2H), 1.76 (br.m, 1H), 2.29 (br.m, 1H), 3.03 (s, 2H), 3.39$3.45(\mathrm{~m}, 2 \mathrm{H}), 7.36-7.38(\mathrm{~m}, 3 \mathrm{H}), 7.61-7.63(\mathrm{~m}, 2 \mathrm{H}), 7.88(\mathrm{~d}, J=8.9 \mathrm{~Hz}, 2 \mathrm{H}), 8.18(\mathrm{~d}, J=8.9$ $\mathrm{Hz}, 2 \mathrm{H}) ;{ }^{13} \mathrm{C}$ NMR $\left(100 \mathrm{MHz}, \mathrm{CDCl}_{3}\right) \delta 23.6,25.1,45.4,46.1,97.5,123.7$ (2C), 125.8, 128.8, 129.0, 129.6, 134.6, 137.4, 148.1, 157.3, 161.0, 168.8; HRMS (ESI) m/z: [M-H] Calcd for $\mathrm{C}_{21} \mathrm{H}_{18} \mathrm{~N}_{3} \mathrm{O}_{5}{ }^{-} 392.1252$; Found 392.1243.

5-Amino-3-(4-chlorophenyl)-5-phenyl-4-(pyrrolidine-1-carbonyl)furan-2(5H)-one (5k)<smiles>NC1(c2ccccc2)OC(=O)C(c2ccc(Cl)cc2)=C1C(=O)N1CCCC1</smiles> 
Compound 5k was obtained according to the general procedure from azirine $\mathbf{1} \mathbf{m}$ and acid $\mathbf{2 d}$ (43 $\mathrm{mg}, 47 \%$, reaction time $100 \mathrm{~h}$ ); column chromatography (petroleum ether/EtOAc $3: 1$ ). Colorless oil. ${ }^{1} \mathrm{H}$ NMR $\left(400 \mathrm{MHz}, \mathrm{CDCl}_{3}\right) \delta 1.17-1.24(\mathrm{~m}, 1 \mathrm{H}), 1.32-1.38(\mathrm{~m}, 1 \mathrm{H}), 1.62-1.67$ (m, 2H), 1.80 (br.s, 1H), 2.34 (br.s, 1H), 2.95 (s, 2H), 3.40-3.52 (m, 2H), 7.37-7.41 (m, 5H), 7.65-7.70 (m, 4H); ${ }^{13} \mathrm{C}$ NMR $\left(100 \mathrm{MHz}, \mathrm{CDCl}_{3}\right) \delta$ 23.8, 25.2, 45.4, 46.1, 97.1, 124.8, 125.9, 126.9, 128.9, 129.1, 129.4, 129.5, 136.1, 138.0, 154.7, 161.8, 169.6; HRMS (ESI) m/z: [M+H] ${ }^{+}$ Calcd for $\mathrm{C}_{21} \mathrm{H}_{20}{ }^{35} \mathrm{ClN}_{2} \mathrm{O}_{3}{ }^{+} 383.1157$; Found 383.1162.

\section{Methyl 5-amino-2-oxo-5-phenyl-4-(pyrrolidine-1-carbonyl)-2,5-dihydrofuran-3- carboxylate $(51)$}<smiles>COC(=O)C1=C(C(=O)N2CCCC2)C(N)(c2ccccc2)OC1=O</smiles>

Compound $\mathbf{5 l}$ was obtained according to the general procedure from azirine $\mathbf{1 m}$ and acid $\mathbf{2 h}$ (31 $\mathrm{mg}$, 40\%, reaction time $48 \mathrm{~h}$ ); column chromatography (petroleum ether/EtOAc 3 : 1). Compound $\mathbf{5 l}$ (20 mg, 25\%) was also obtained from azirine $\mathbf{1 m}$, acid $\mathbf{2 h}$ and $\mathrm{K}_{2} \mathrm{CO}_{3}$ in $\mathrm{MeCN}$ at rt. Colorless oil. ${ }^{1} \mathrm{H}$ NMR (400 MHz, $\left.\mathrm{CDCl}_{3}\right) \delta 1.32-1.43(\mathrm{~m}, 1 \mathrm{H}), 1.61-1.86(\mathrm{~m}, 4 \mathrm{H}), 2.87-2.91$ (m, 3H), 3.43-3.49 (m, 1H), 3.56-3.62 (m, 1H), $3.86(\mathrm{~s}, 3 \mathrm{H}), 7.41-7.43(\mathrm{~m}, 3 \mathrm{H}), 7.61-7.63(\mathrm{~m}$, $2 \mathrm{H}) ;{ }^{13} \mathrm{C} \mathrm{NMR}\left(100 \mathrm{MHz}, \mathrm{CDCl}_{3}\right) \delta 23.9,25.4,45.4,46.4,52.9,97.1,118.3,126.3,129.0$, 130.0, 136.5, 159.9, 160.2, 165.6, 167.9; HRMS (ESI) m/z: $[\mathrm{M}+\mathrm{Na}]^{+} \mathrm{Calcd}$ for $\mathrm{C}_{17} \mathrm{H}_{18} \mathrm{~N}_{2} \mathrm{NaO}_{5}{ }^{+}$ 353.1108; Found 353.1100.

Methyl 2-amino-3-benzoyl-4-(4-nitrophenyl)-5-oxo-2,5-dihydrofuran-2-carboxylate (5m)

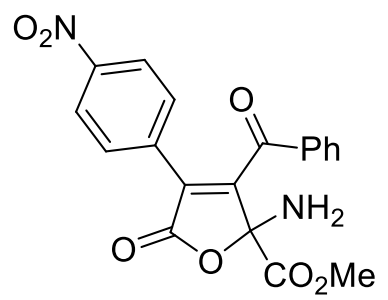

Compound 5m was obtained according to the general procedure from azirine 1r and acid 2a (72 $\mathrm{mg}, 80 \%$, reaction time $24 \mathrm{~h}$ ); column chromatography (petroleum ether/EtOAc $3: 1$ ). Colorless solid; mp 126-127 ${ }^{\circ} \mathrm{C}$ (Et $2 \mathrm{O} /$ hexane). ${ }^{1} \mathrm{H}$ NMR (400 MHz, $\mathrm{CDCl}_{3}$ ) $\delta 3.04$ (br.s, 2H), 3.87 (s, $3 \mathrm{H}), 7.32(\mathrm{t}, J=7.8 \mathrm{~Hz}, 2 \mathrm{H}), 7.81(\mathrm{t}, J=7.4 \mathrm{~Hz}, 1 \mathrm{H}), 7.66(\mathrm{~d}, J=8.8 \mathrm{~Hz}, 2 \mathrm{H}), 7.73(\mathrm{~d}, J=7.4$ $\mathrm{Hz}, 2 \mathrm{H}), 8.06(\mathrm{~d}, J=8.8 \mathrm{~Hz}, 2 \mathrm{H}) ;{ }^{13} \mathrm{C} \mathrm{NMR}\left(100 \mathrm{MHz}, \mathrm{CDCl}_{3}\right) \delta 54.1,93.9,123.5,129.0$, 129.2, 130.3, 131.6, 133.8, 133.9, 135.1, 148.3, 153.7, 165.5, 168.2, 190.5; HRMS (ESI) m/z: [M-H] $]^{-}$Calcd for $\mathrm{C}_{19} \mathrm{H}_{13} \mathrm{~N}_{2} \mathrm{O}_{7}{ }^{-} 381.0728$; Found 381.0719. 


\section{Synthesis of 4-bromoisoxazole 12d,h,n,p}

4-Bromoisoxazoles 12d,h,n,p were synthesized using $N$-bromosuccinimide according to the reported procedure. ${ }^{7}$

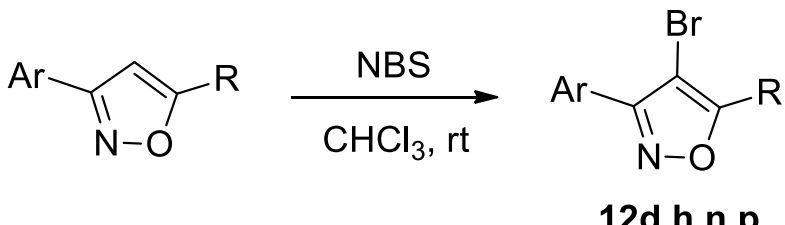

\section{4-Bromo-3-(4-fluorophenyl)-5-methoxyisoxazole (12d)}<smiles>COc1onc(-c2ccc(F)cc2)c1Br</smiles>

Colorless solid (466 mg, 81\%); mp 59-60 ${ }^{\circ} \mathrm{C}\left(\mathrm{Et}_{2} \mathrm{O} /\right.$ hexane). ${ }^{1} \mathrm{H}$ NMR (400 MHz, $\left.\mathrm{CDCl}_{3}\right) \delta 4.24$ (s, 3H), 7.17-7.21 (m, 2H), 7.84-7.87 (m, 2H); $\left.{ }^{13} \mathrm{C} \mathrm{NMR} \mathrm{(100} \mathrm{MHz,} \mathrm{CDCl}_{3}\right) \delta 58.5,66.6,115.8$ $(\mathrm{d}, J=21.8 \mathrm{~Hz}), 124.4(\mathrm{~d}, J=3.5 \mathrm{~Hz}), 130.0(\mathrm{~d}, J=8.5 \mathrm{~Hz}), 161.7,164.0(\mathrm{~d}, J=250.6 \mathrm{~Hz})$, 169.4; HRMS (ESI) m/z: [M+H] ${ }^{+}$Calcd for $\mathrm{C}_{10} \mathrm{H}_{8}{ }^{79} \mathrm{BrFNO}_{2}{ }^{+} 271.9717$; Found 271.9718.

\section{4-Bromo-3-(3,4-dimethoxyphenyl)-5-methoxyisoxazole (12h)}<smiles>COc1ccc(-c2noc(OC)c2Br)cc1OC</smiles>

Colorless solid (510 mg, 86\%); mp 94-96 ${ }^{\circ} \mathrm{C}\left(\mathrm{Et}_{2} \mathrm{O} / \mathrm{hexane}\right) .{ }^{1} \mathrm{H}$ NMR (400 MHz, $\left.\mathrm{CDCl}_{3}\right) \delta 3.94$ $(\mathrm{s}, 3 \mathrm{H}), 3.95(\mathrm{~s}, 3 \mathrm{H}), 4.21(\mathrm{~s}, 3 \mathrm{H}), 6.97(\mathrm{~d}, J=8.4 \mathrm{~Hz}, 1 \mathrm{H}), 7.38(\mathrm{~d}, J=1.9 \mathrm{~Hz}, 1 \mathrm{H}), 7.49$ (dd, $J$ $=8.3$ and $2.0 \mathrm{~Hz}, 1 \mathrm{H}) ;{ }^{13} \mathrm{C} \mathrm{NMR}\left(100 \mathrm{MHz}, \mathrm{CDCl}_{3}\right) \delta 55.9(2 \mathrm{C}), 58.4,66.6,110.6,110.9,120.8$, 120.9, 149.0, 150.7, 161.9, 169.2; HRMS (ESI) m/z: $[\mathrm{M}+\mathrm{H}]^{+}$Calcd for $\mathrm{C}_{12} \mathrm{H}_{13}{ }^{79} \mathrm{BrNO}_{4}{ }^{+}$ 314.0022; Found 314.0026.

\section{$N$-Benzyl-4-bromo- $N$-methyl-3-phenylisoxazol-5-amine (12n)}<smiles>CN(Cc1ccccc1)c1onc(-c2ccccc2)c1Br</smiles>

Colorless oil (452 mg, 75\%). ${ }^{1} \mathrm{H}$ NMR (400 MHz, $\left.\mathrm{CDCl}_{3}\right) \delta 3.15(\mathrm{~s}, 3 \mathrm{H}), 4.78(\mathrm{~s}, 2 \mathrm{H}), 7.33-7.43$ $(\mathrm{m}, 5 \mathrm{H}), 7.49-7.51(\mathrm{~m}, 3 \mathrm{H}), 7.78-7.81(\mathrm{~m}, 2 \mathrm{H}) ;{ }^{13} \mathrm{C} \mathrm{NMR}\left(100 \mathrm{MHz}, \mathrm{CDCl}_{3}\right) \delta 36.4,54.9,65.5$, 127.8 (2C), 128.4, 128.5, 128.7, 128.8, 129.8, 136.6, 163.1, 166.3; HRMS (ESI) m/z: [M+H] ${ }^{+}$ Calcd for $\mathrm{C}_{17} \mathrm{H}_{16}{ }^{79} \mathrm{BrN}_{2} \mathrm{O}^{+} 343.0441$; Found 343.0441. 


\section{4-Bromo-5-(tert-butoxy)-3-phenylisoxazole (12p)}

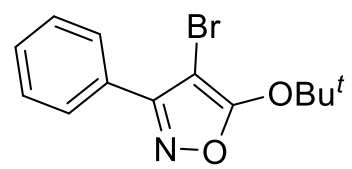

Yellow oil (1.2 g, 85\%). ${ }^{1} \mathrm{H}$ NMR (400 MHz, $\left.\mathrm{CDCl}_{3}\right) \delta 1.60(\mathrm{~s}, 9 \mathrm{H}), 7.49-7.51(\mathrm{~m}, 3 \mathrm{H}), 7.87-$ $7.89(\mathrm{~m}, 2 \mathrm{H}) ;{ }^{13} \mathrm{C} \mathrm{NMR}\left(100 \mathrm{MHz}, \mathrm{CDCl}_{3}\right) \delta 28.8,74.5,88.1,127.8,128.6(2 \mathrm{C}), 130.1,161.6$, 169.2; HRMS (ESI) m/z: [M+Na] ${ }^{+}$Calcd for $\mathrm{C}_{13} \mathrm{H}_{14}{ }^{79} \mathrm{BrNNaO}_{2}{ }^{+}$318.0100; Found 318.0101. 


\section{NMR spectra of new compounds}

${ }^{1} \mathrm{H}\left(400 \mathrm{MHz}, \mathrm{CDCl}_{3}\right)$ and ${ }^{13} \mathrm{C}\left(100 \mathrm{MHz}, \mathrm{CDCl}_{3}\right)$ NMR spectra of compound 1d

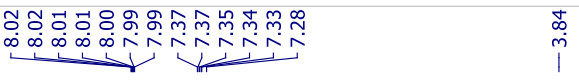<smiles>CC(=O)C1(Br)N=C1c1ccc(F)cc1</smiles>

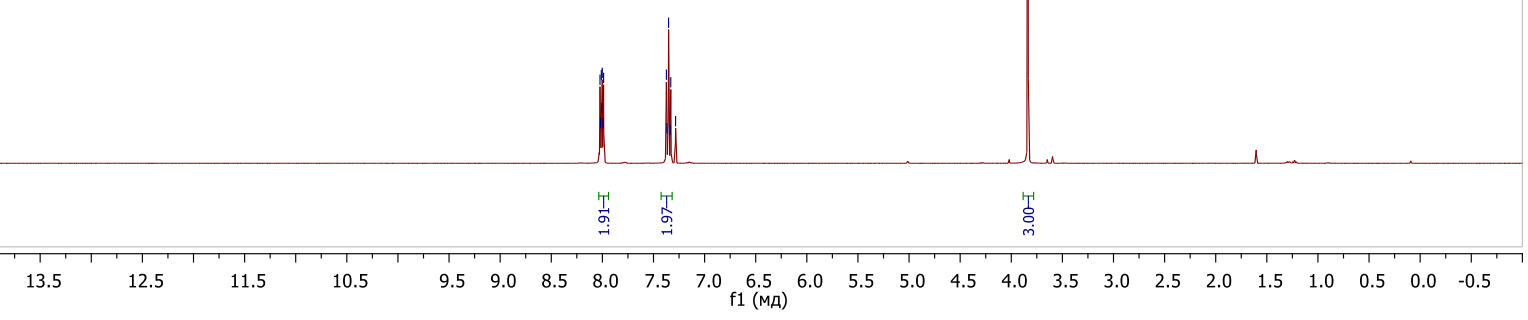

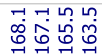

1,

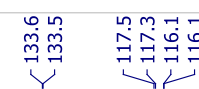

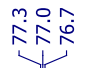

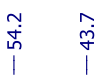

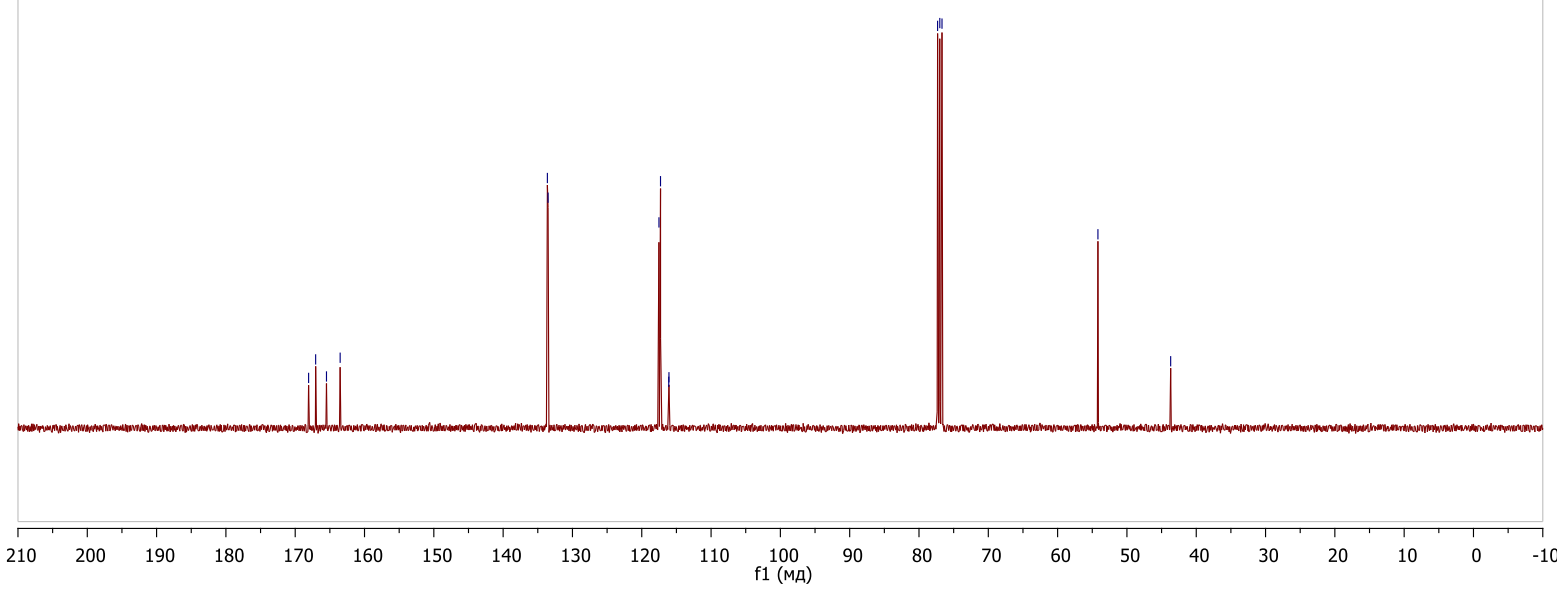


${ }^{1} \mathrm{H}\left(400 \mathrm{MHz}, \mathrm{CDCl}_{3}\right)$ and ${ }^{13} \mathrm{C}\left(100 \mathrm{MHz}, \mathrm{CDCl}_{3}\right)$ NMR spectra of compound $\mathbf{1 n}$

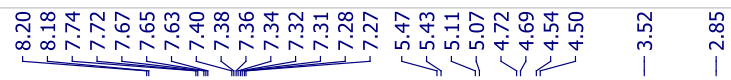<smiles>CN(Cc1ccccc1)C(=O)C1(Br)N=C1c1ccccc1</smiles>
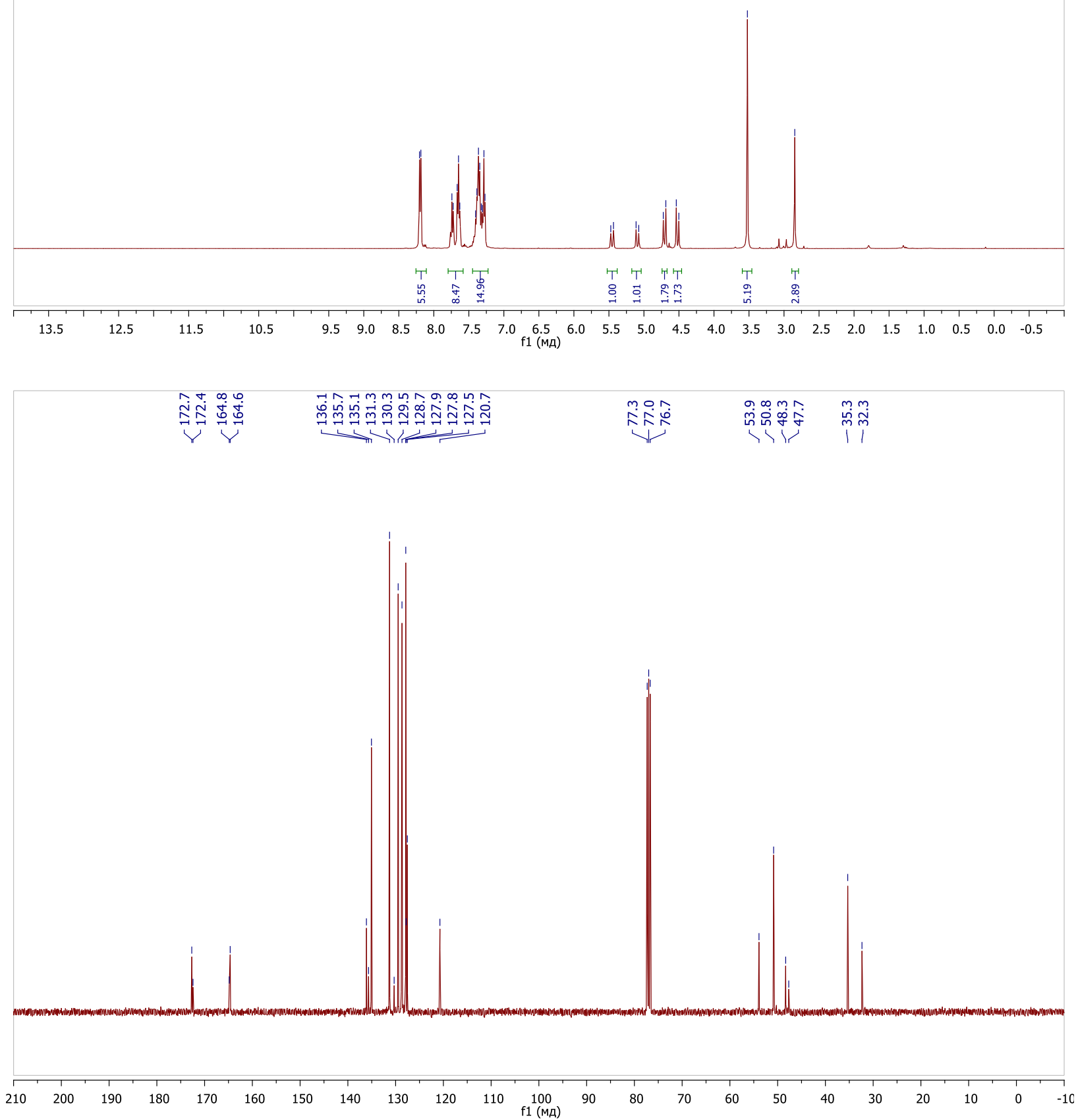
${ }^{1} \mathrm{H}\left(400 \mathrm{MHz}, \mathrm{CDCl}_{3}\right)$ and ${ }^{13} \mathrm{C}\left(100 \mathrm{MHz}, \mathrm{CDCl}_{3}\right)$ NMR spectra of compound $\mathbf{1 p}$
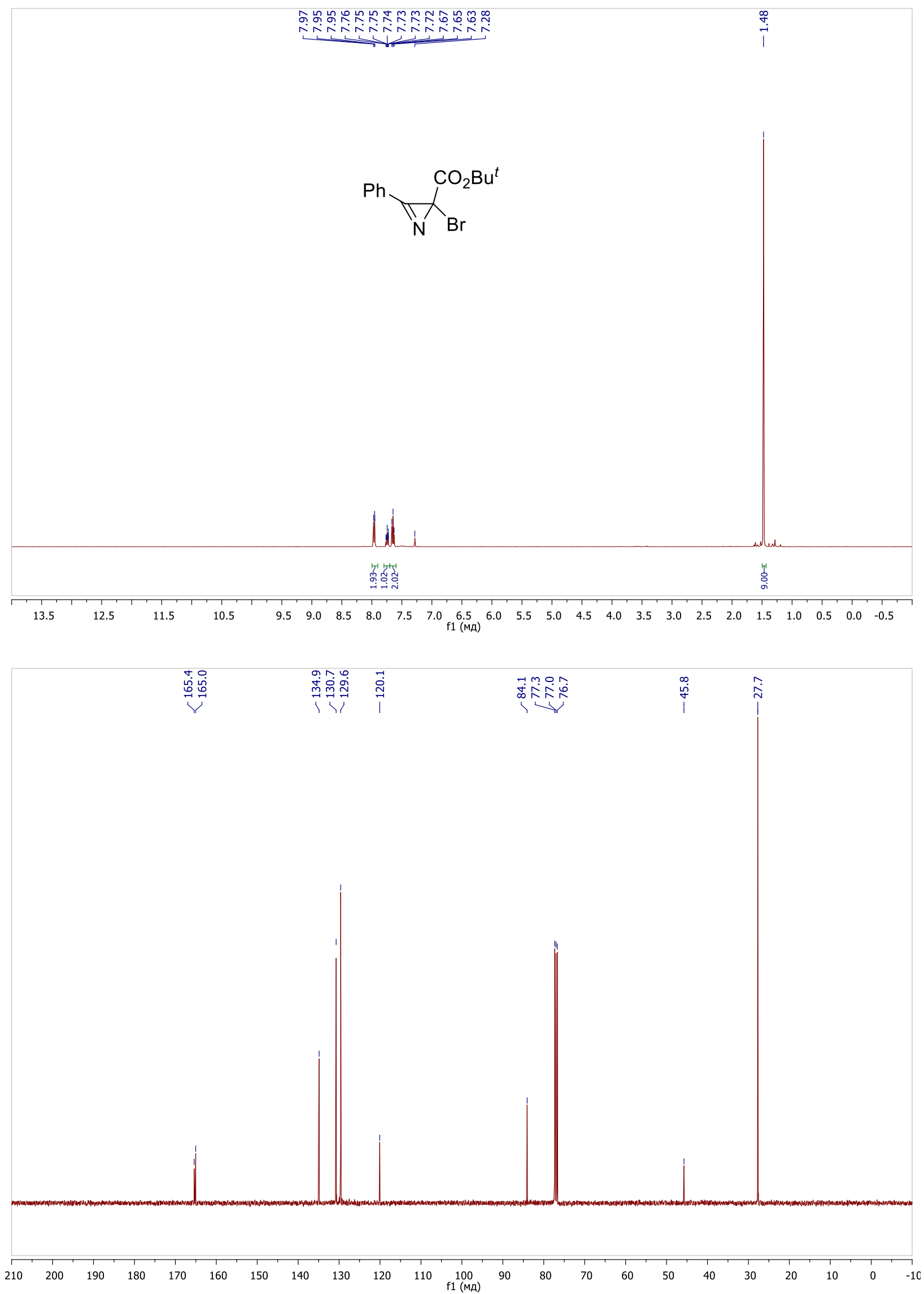
${ }^{1} \mathrm{H}\left(400 \mathrm{MHz}, \mathrm{CDCl}_{3}\right)$ and ${ }^{13} \mathrm{C}\left(100 \mathrm{MHz}, \mathrm{CDCl}_{3}\right)$ NMR spectra of compound $\mathbf{3 c}$

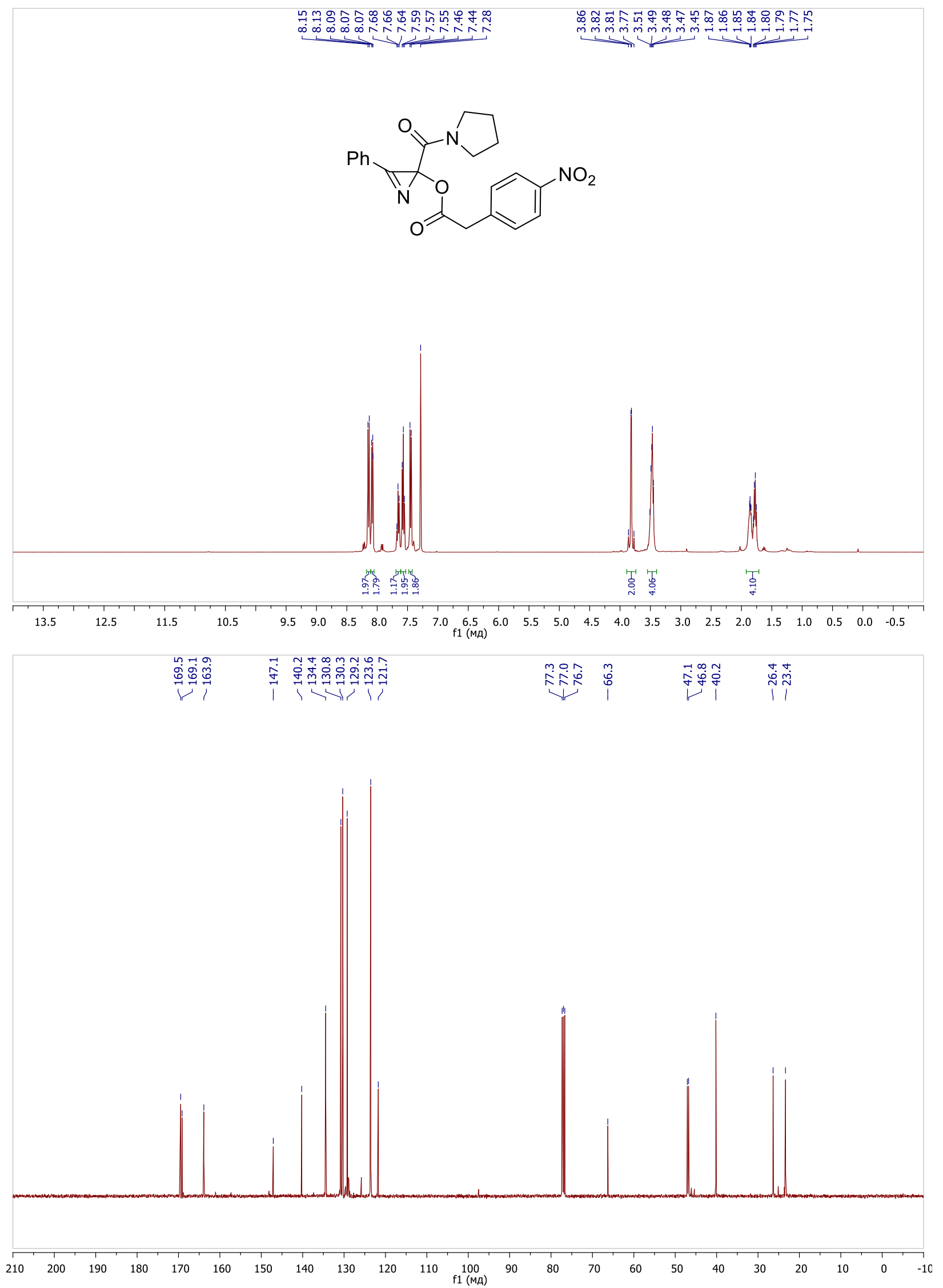


${ }^{1} \mathrm{H}\left(400 \mathrm{MHz}, \mathrm{CDCl}_{3}\right)$ and ${ }^{13} \mathrm{C}\left(100 \mathrm{MHz}, \mathrm{CDCl}_{3}\right) \mathrm{NMR}$ spectra of compound $4 \mathbf{a}$
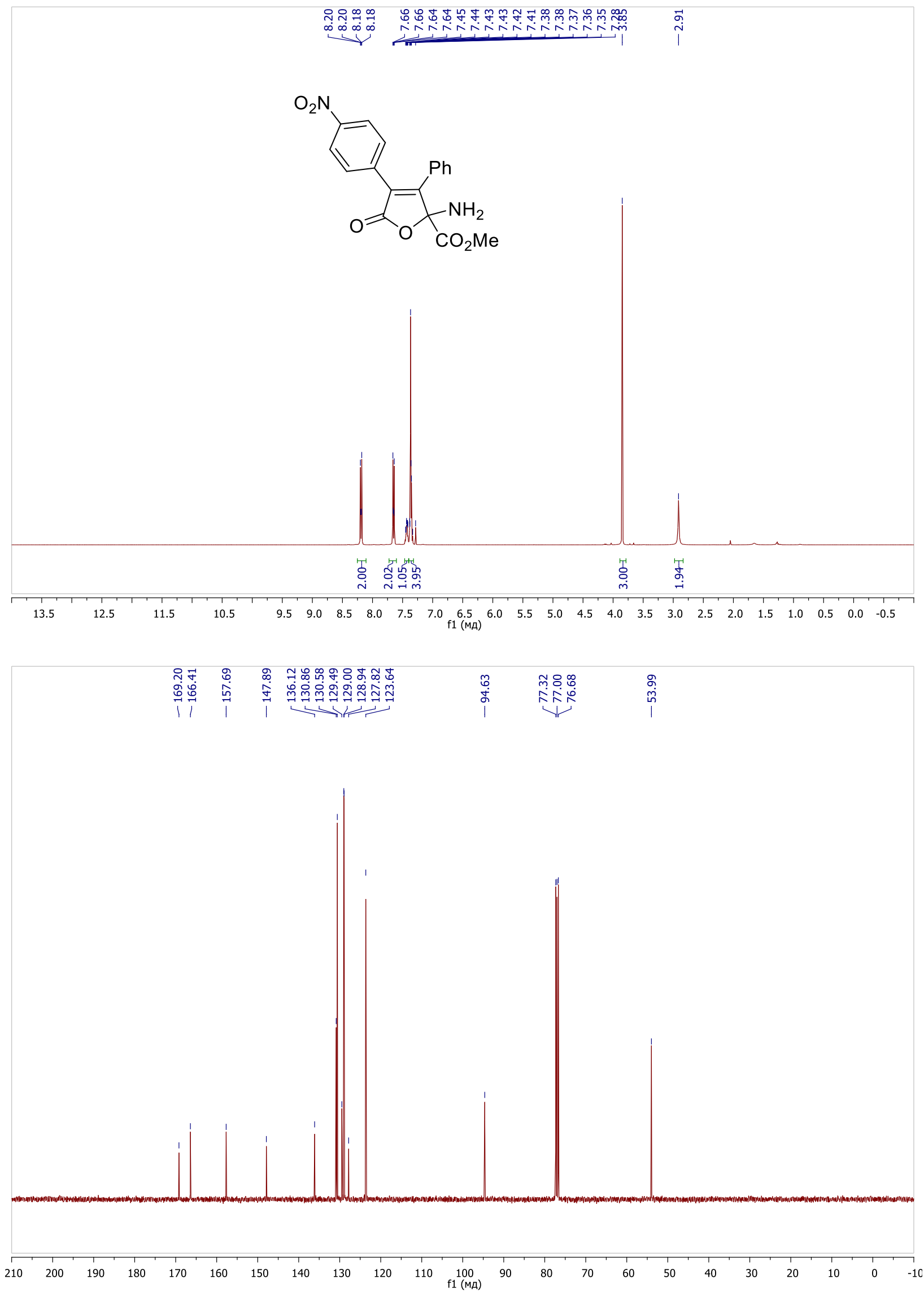
HSQC ${ }^{1} \mathrm{H}_{-}{ }^{13} \mathrm{C}$ and $\mathrm{HMBC}{ }^{1} \mathrm{H}_{-}{ }^{13} \mathrm{C}$ NMR spectra $\left(\mathrm{CDCl}_{3}\right)$ of compound 4a
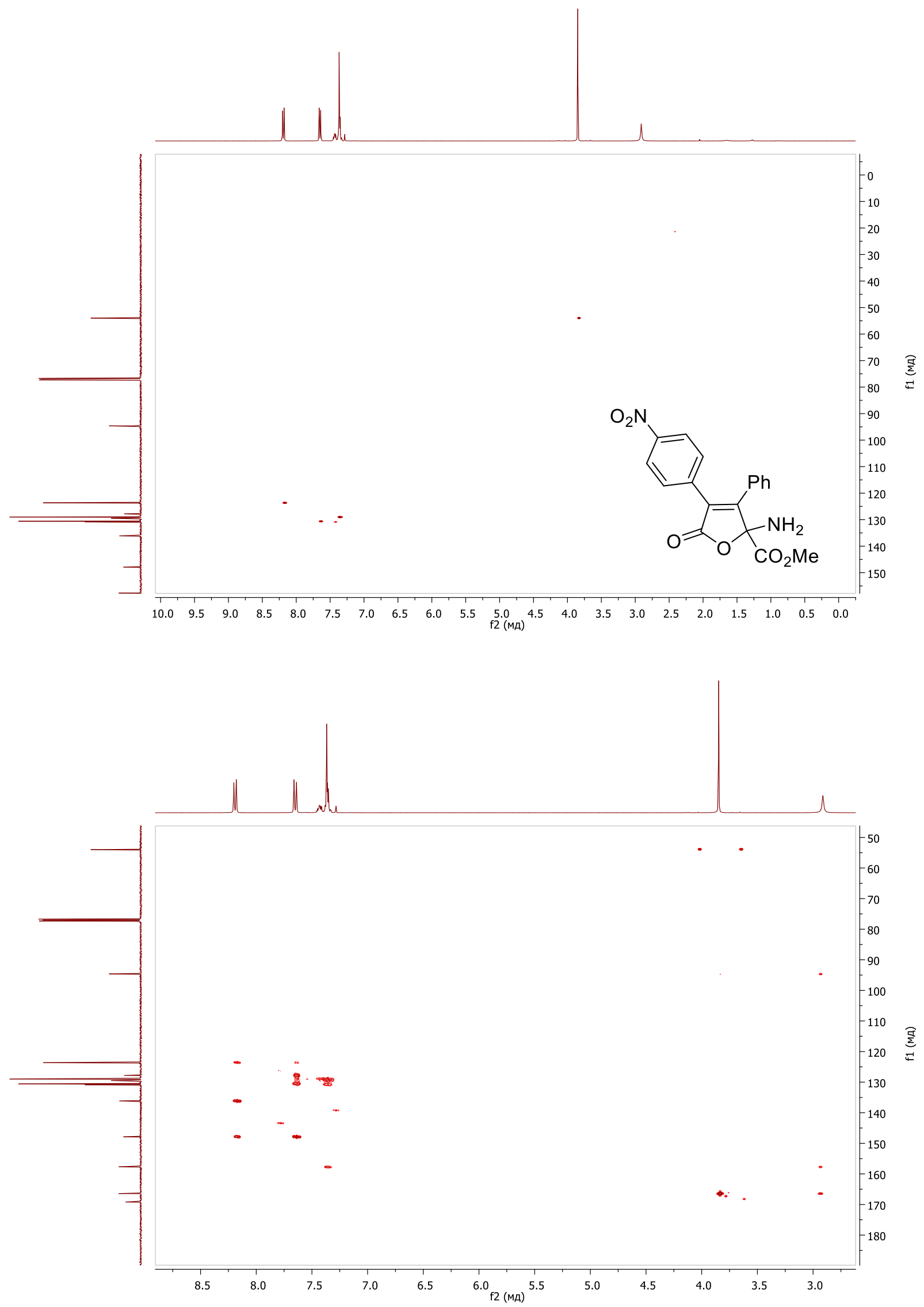
HMBC ${ }^{1} \mathrm{H}^{-15} \mathrm{~N}$ NMR spectrum $\left(\mathrm{CDCl}_{3}\right)$ of compound $\mathbf{4 a}$

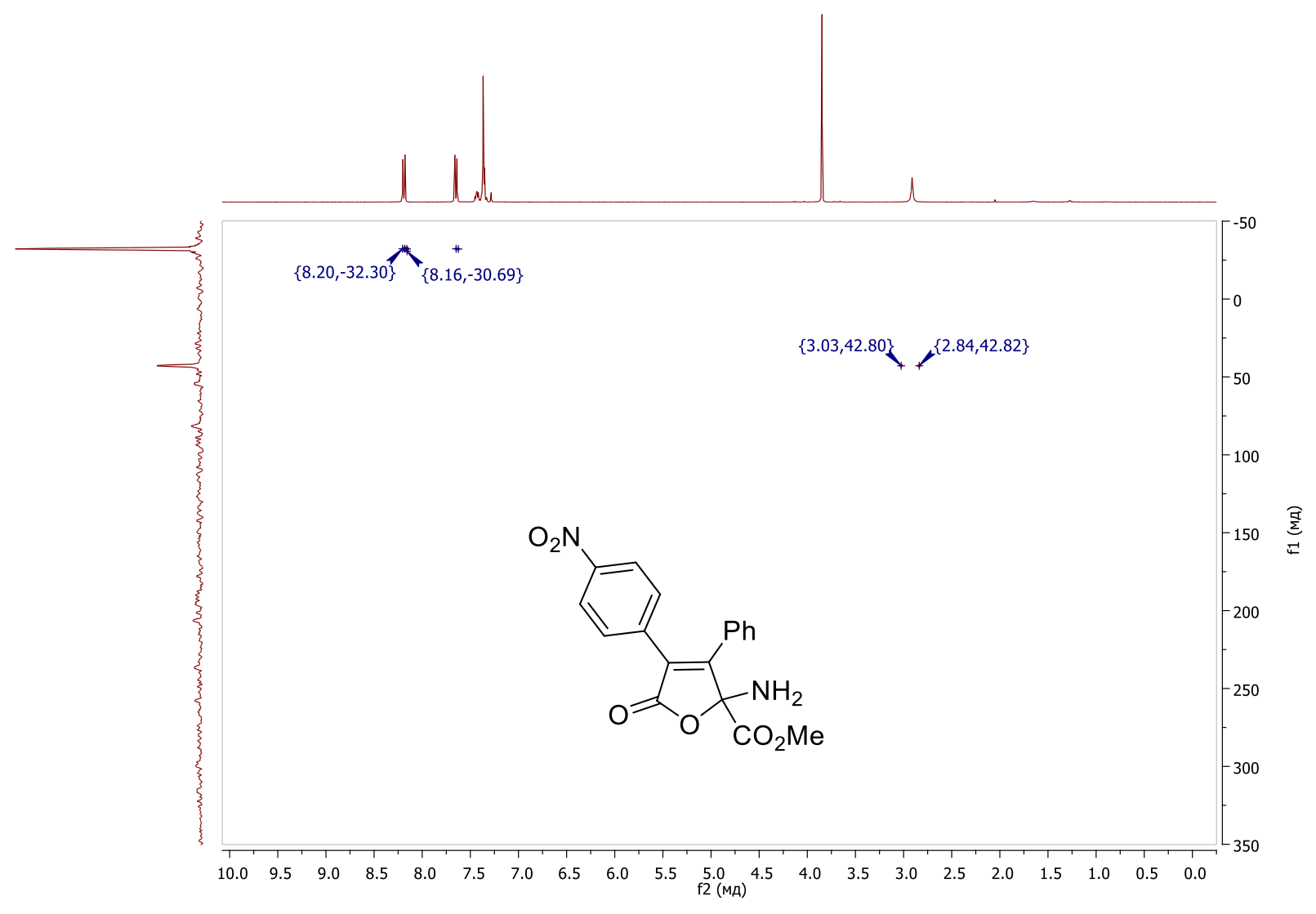


${ }^{1} \mathrm{H}\left(400 \mathrm{MHz}, \mathrm{CDCl}_{3}-\mathrm{DMSO}-\mathrm{d}_{6}\right.$ mixture $)$ and ${ }^{13} \mathrm{C}\left(100 \mathrm{MHz}, \mathrm{CDCl}_{3}-\mathrm{DMSO}_{6}-\mathrm{d}_{6}\right.$ mixture $)$ NMR spectra of compound $\mathbf{4 b}$
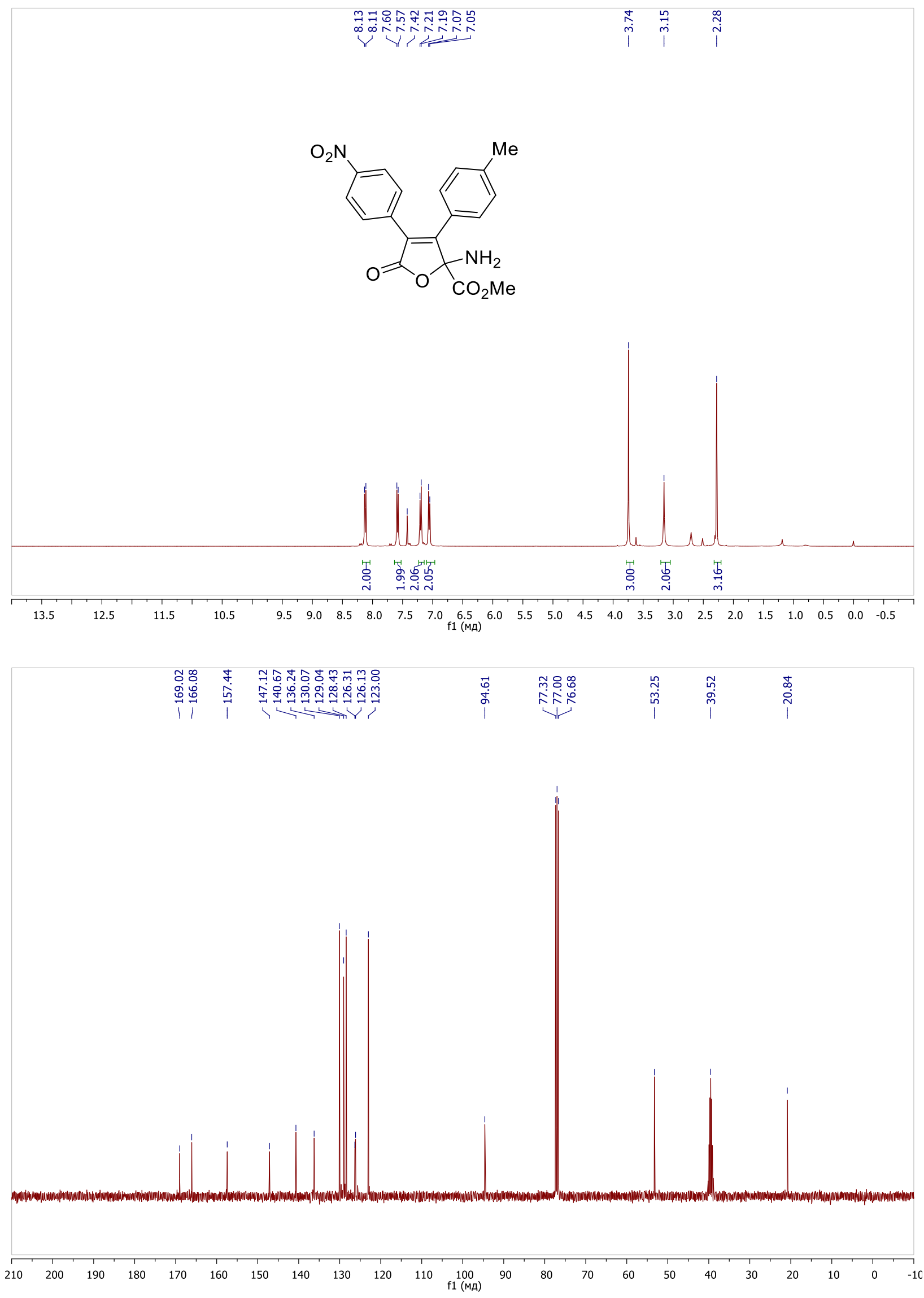
${ }^{1} \mathrm{H}\left(400 \mathrm{MHz}, \mathrm{CDCl}_{3}\right)$ and ${ }^{13} \mathrm{C}\left(100 \mathrm{MHz}, \mathrm{CDCl}_{3}\right)$ NMR spectra of compound $4 \mathbf{c}$
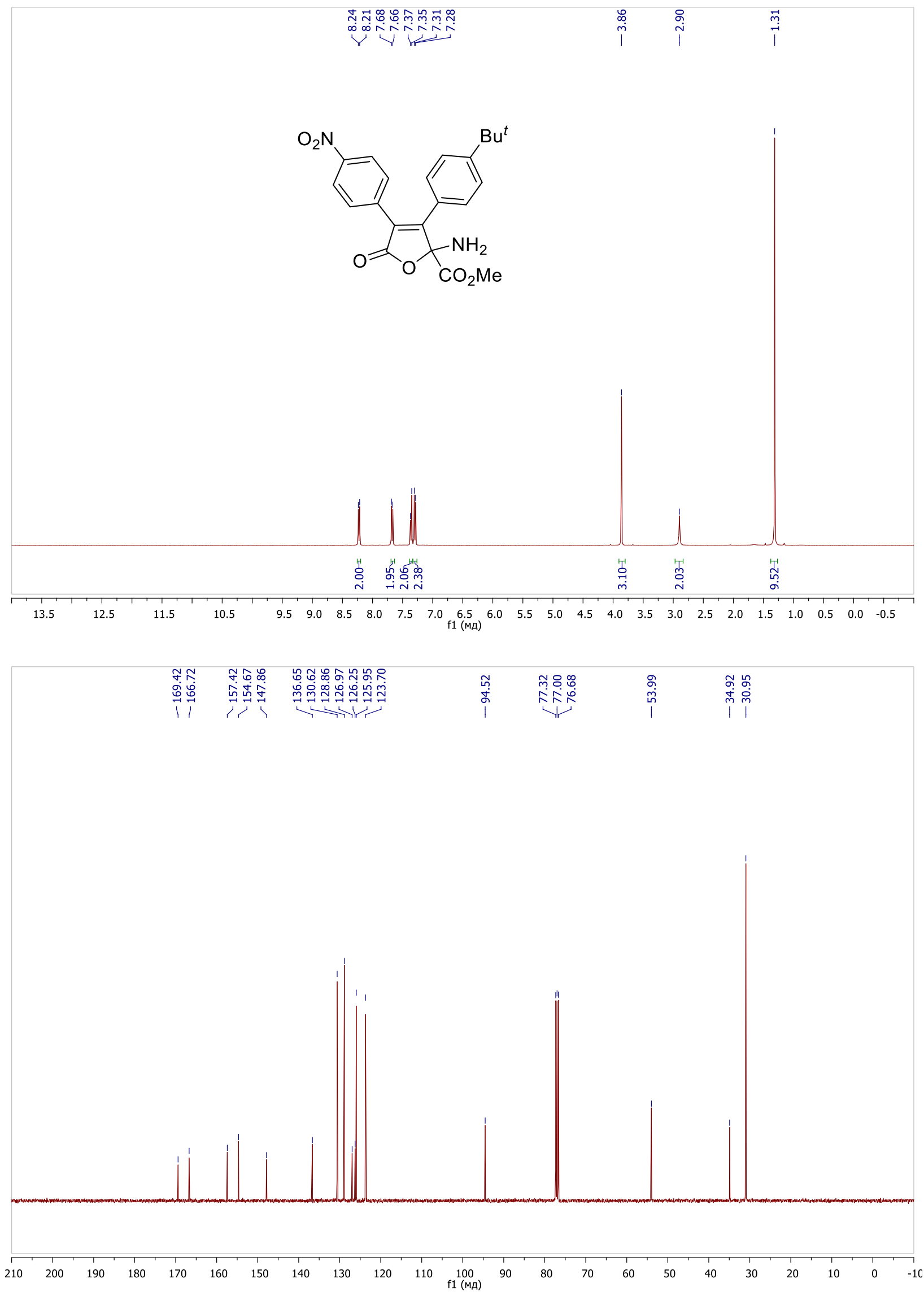
${ }^{1} \mathrm{H}\left(400 \mathrm{MHz}, \mathrm{CDCl}_{3}-\mathrm{DMSO}-\mathrm{d}_{6}\right.$ mixture $)$ and ${ }^{13} \mathrm{C}\left(100 \mathrm{MHz}, \mathrm{CDCl}_{3}-\mathrm{DMSO}_{6}-\mathrm{d}_{6}\right.$ mixture $)$ NMR spectra of compound $\mathbf{4 d}$
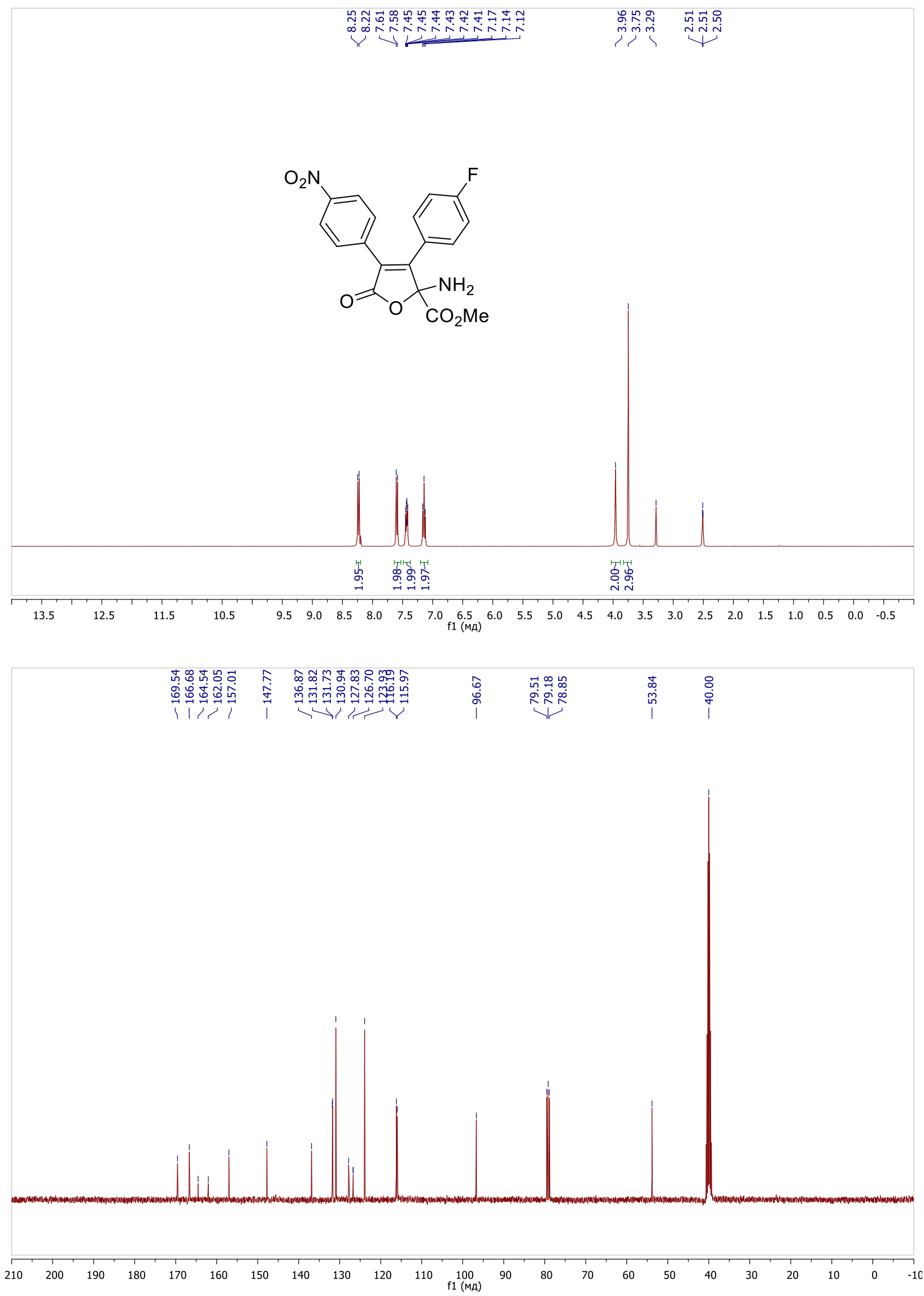
${ }^{1} \mathrm{H}\left(400 \mathrm{MHz}, \mathrm{CDCl}_{3}-\mathrm{DMSO}-\mathrm{d}_{6}\right.$ mixture $)$ and ${ }^{13} \mathrm{C}\left(100 \mathrm{MHz}, \mathrm{CDCl}_{3}-\mathrm{DMSO}_{6}-\mathrm{d}_{6}\right.$ mixture $)$ NMR spectra of compound $\mathbf{4 e}$
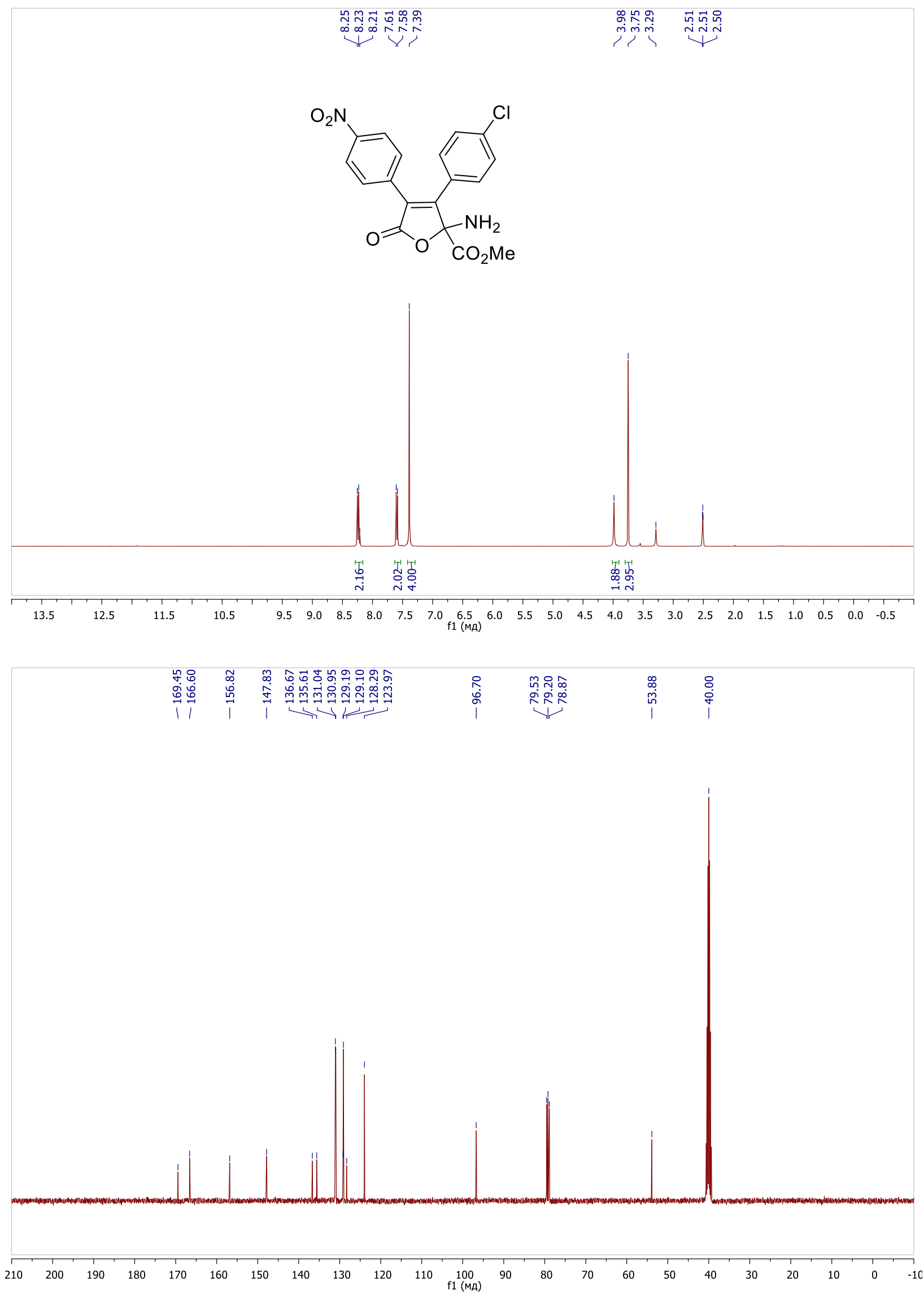
${ }^{1} \mathrm{H}\left(400 \mathrm{MHz}, \mathrm{CDCl}_{3}-\mathrm{DMSO}-\mathrm{d}_{6}\right.$ mixture $)$ and ${ }^{13} \mathrm{C}\left(100 \mathrm{MHz}, \mathrm{CDCl}_{3}-\mathrm{DMSO}_{6}-\mathrm{d}_{6}\right.$ mixture $)$ NMR spectra of compound $\mathbf{4 f}$
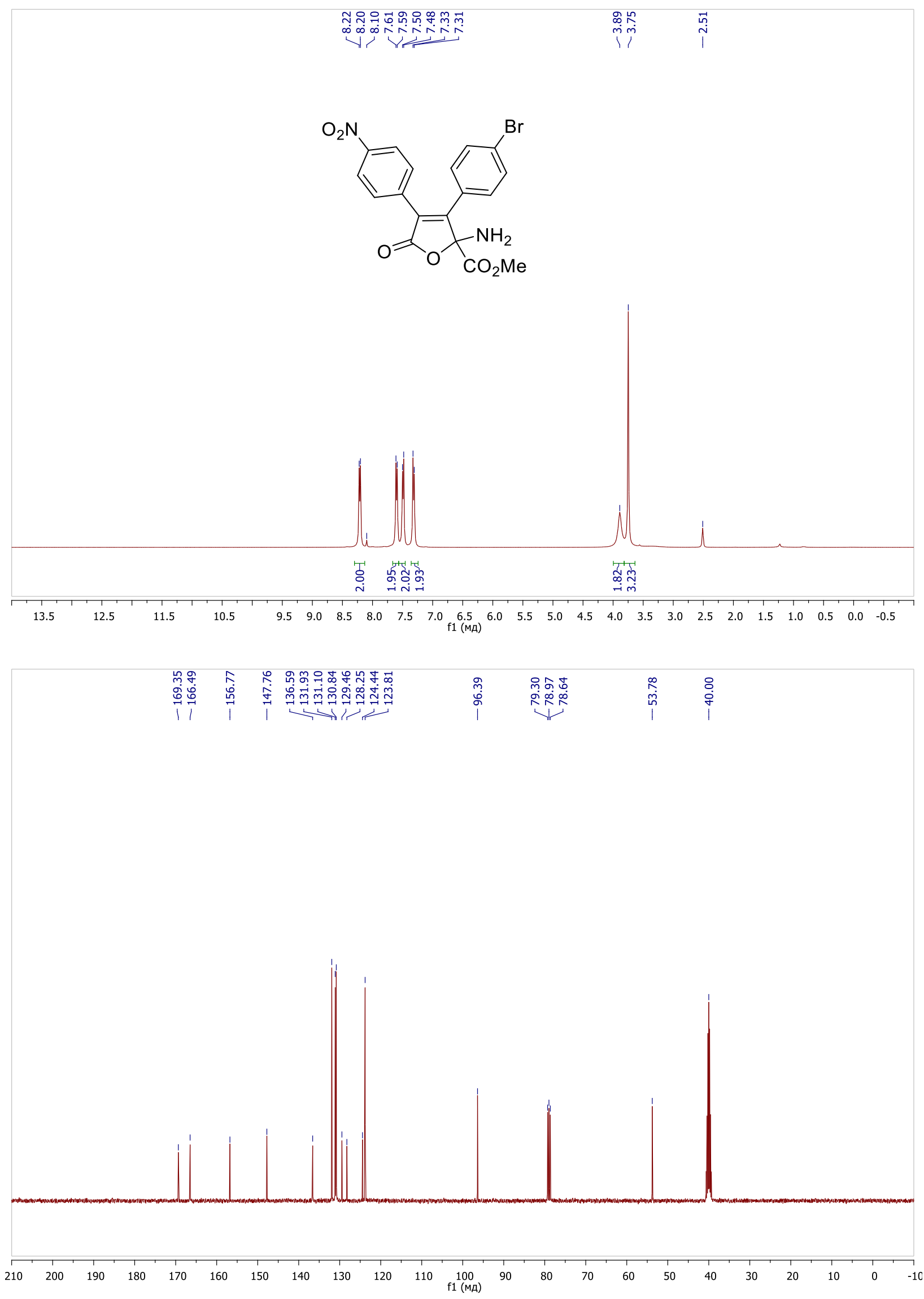
${ }^{1} \mathrm{H}\left(400 \mathrm{MHz}, \mathrm{CDCl}_{3}\right)$ and ${ }^{13} \mathrm{C}\left(100 \mathrm{MHz}, \mathrm{CDCl}_{3}\right) \mathrm{NMR}$ spectra of compound $\mathbf{4 g}$
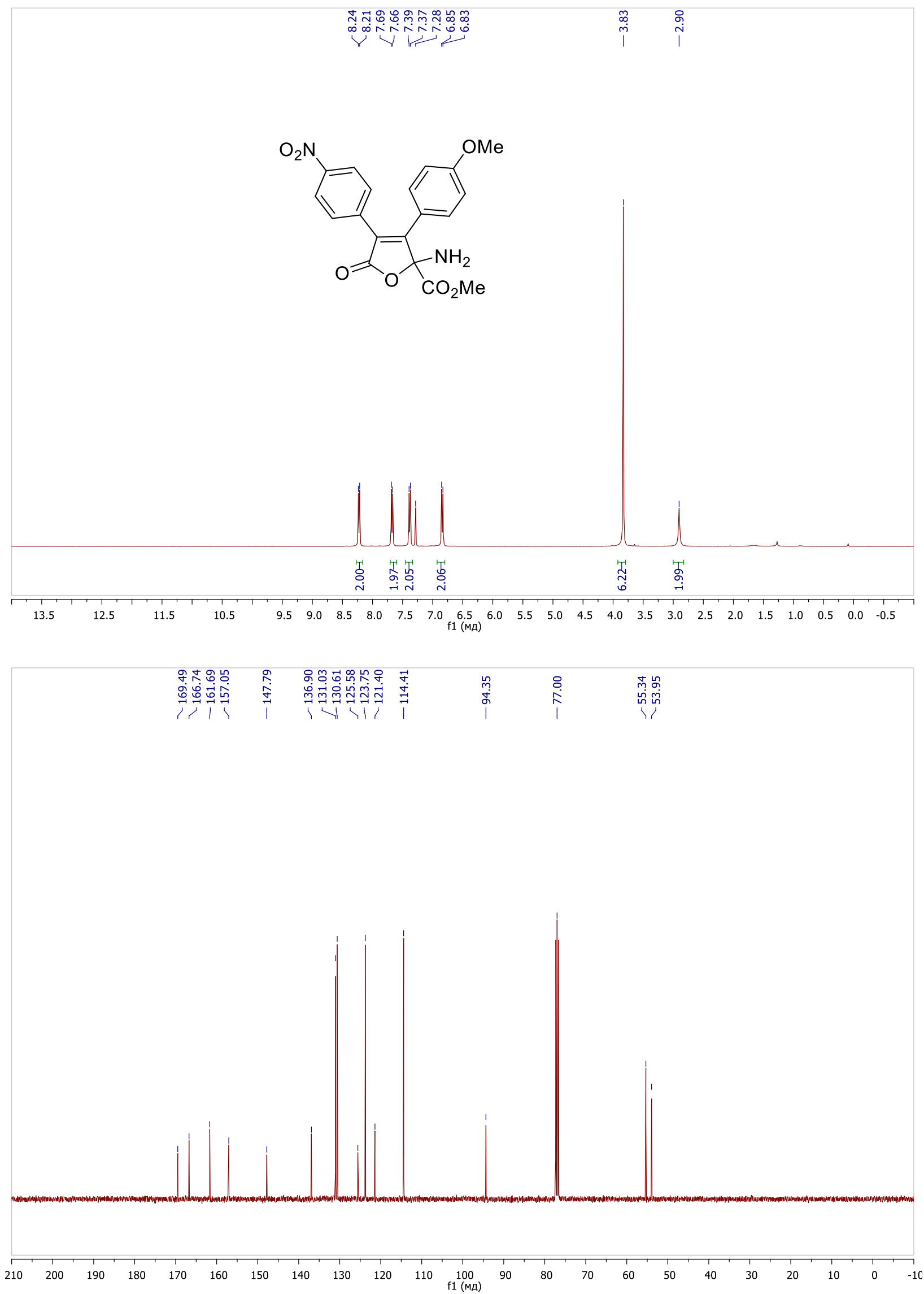
${ }^{1} \mathrm{H}\left(400 \mathrm{MHz}, \mathrm{CDCl}_{3}\right)$ and ${ }^{13} \mathrm{C}\left(100 \mathrm{MHz}, \mathrm{CDCl}_{3}\right) \mathrm{NMR}$ spectra of compound $\mathbf{4 h}$
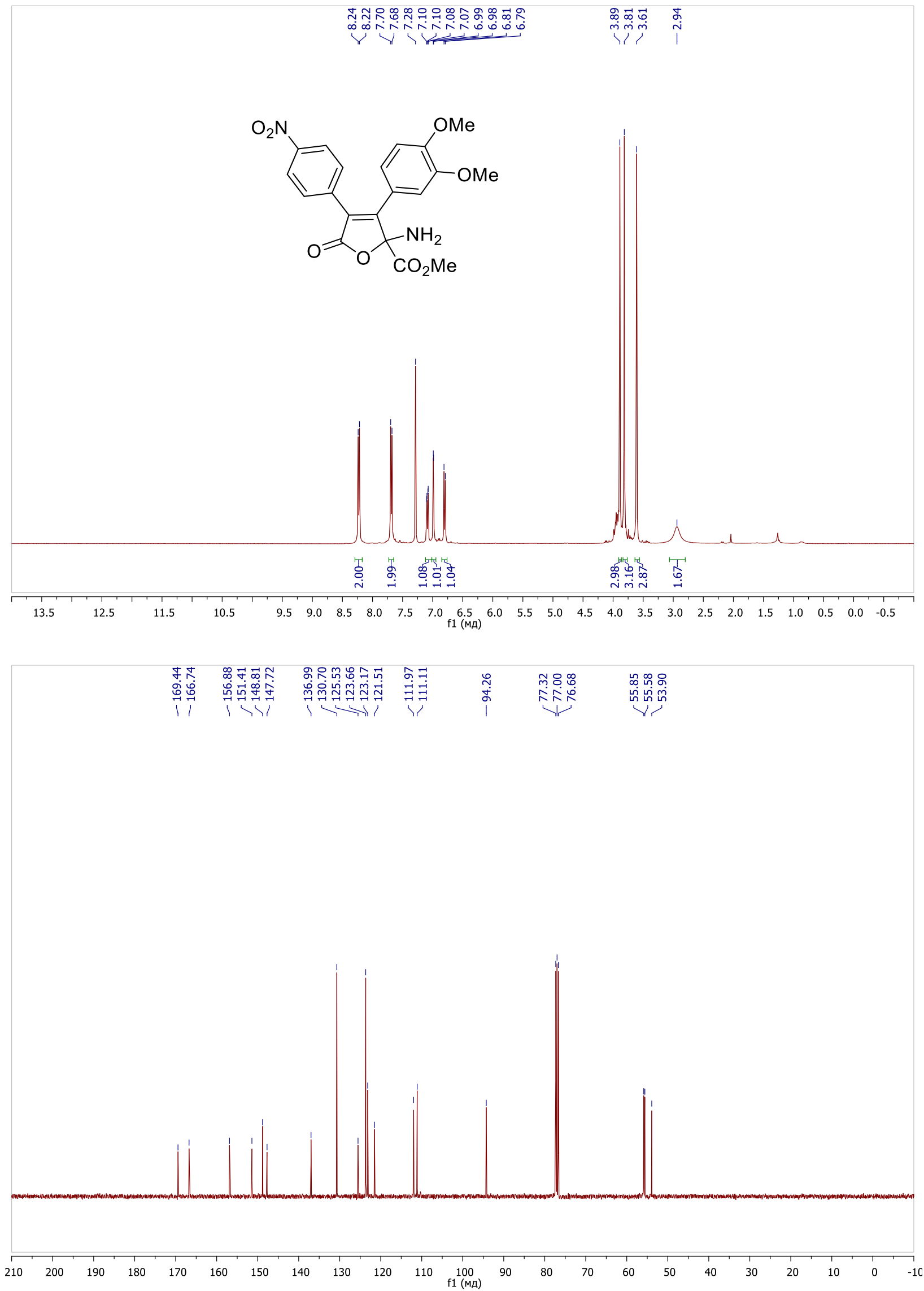
${ }^{1} \mathrm{H}\left(400 \mathrm{MHz}, \mathrm{CDCl}_{3}\right)$ and ${ }^{13} \mathrm{C}\left(100 \mathrm{MHz}, \mathrm{CDCl}_{3}\right)$ NMR spectra of compound $\mathbf{4 i}$
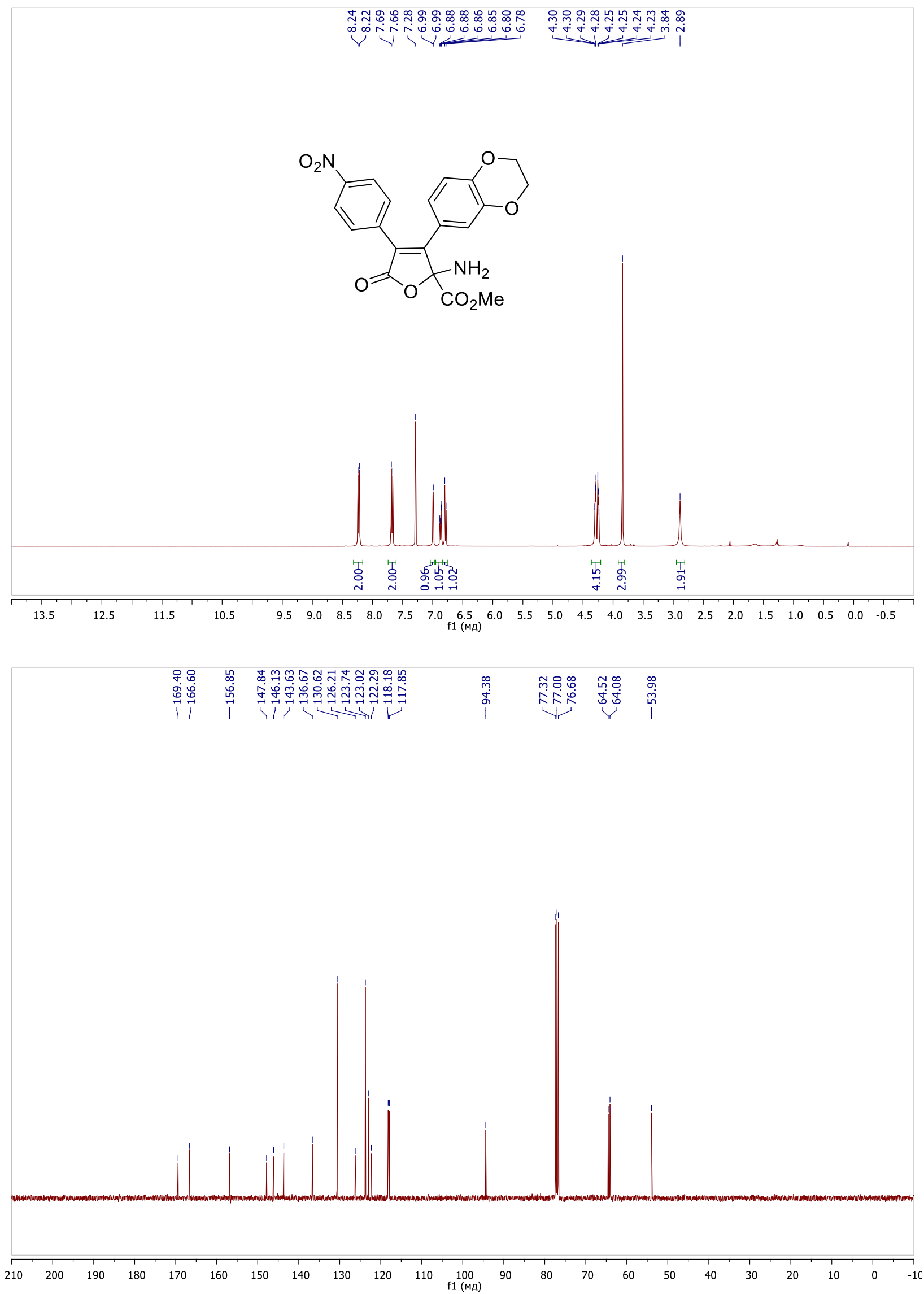
${ }^{1} \mathrm{H}\left(400 \mathrm{MHz}, \mathrm{CDCl}_{3}-\right.$ DMSO-d $\mathrm{d}_{6}$ mixture $)$ and ${ }^{13} \mathrm{C}\left(100 \mathrm{MHz}, \mathrm{CDCl}_{3}-\right.$ DMSO- $\mathrm{d}_{6}$ mixture $)$ NMR $4 \mathbf{j}$
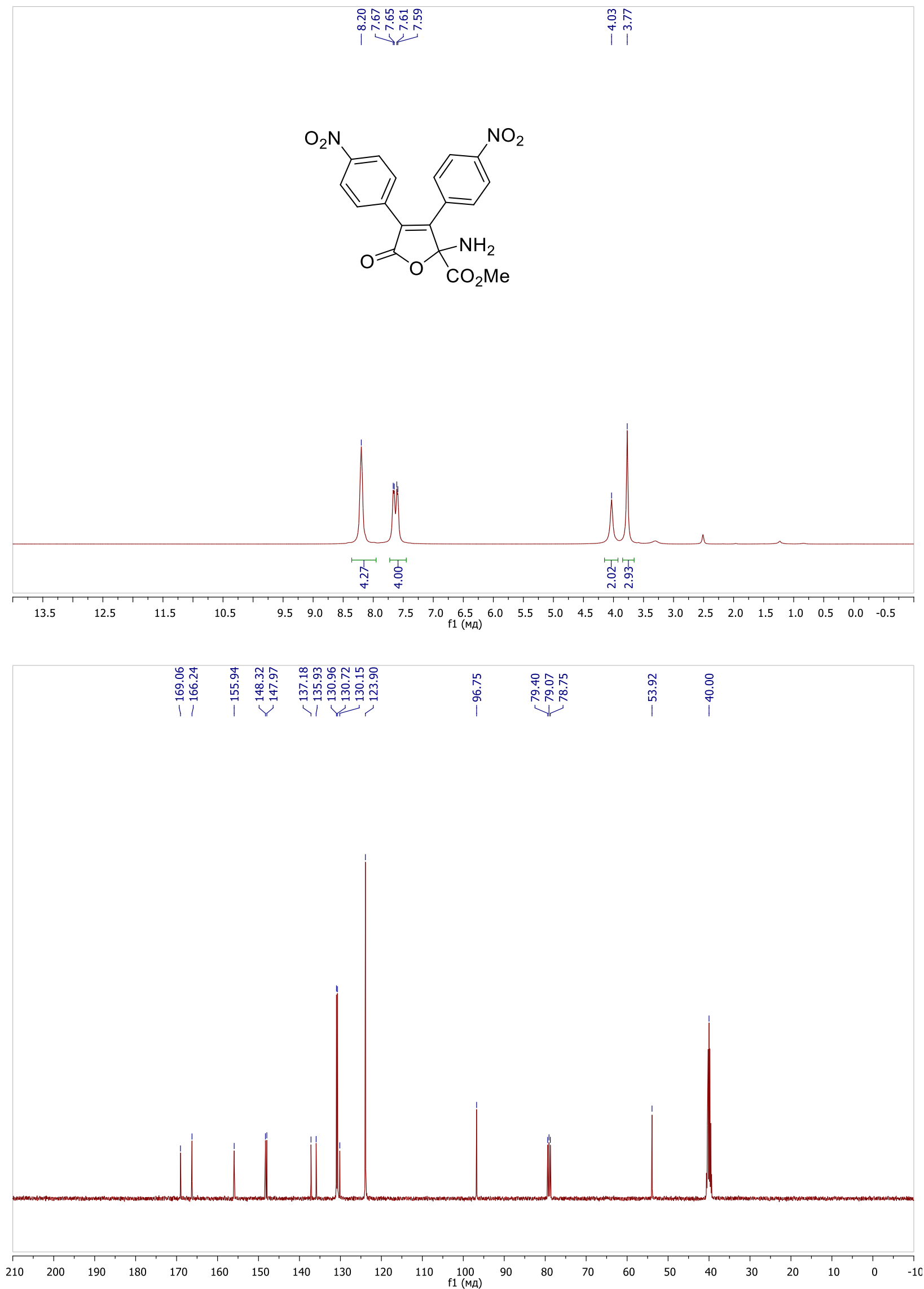
${ }^{1} \mathrm{H}\left(400 \mathrm{MHz}, \mathrm{CDCl}_{3}\right)$ and ${ }^{13} \mathrm{C}\left(100 \mathrm{MHz}, \mathrm{CDCl}_{3}\right) \mathrm{NMR}$ spectra of compound $\mathbf{4 k}$
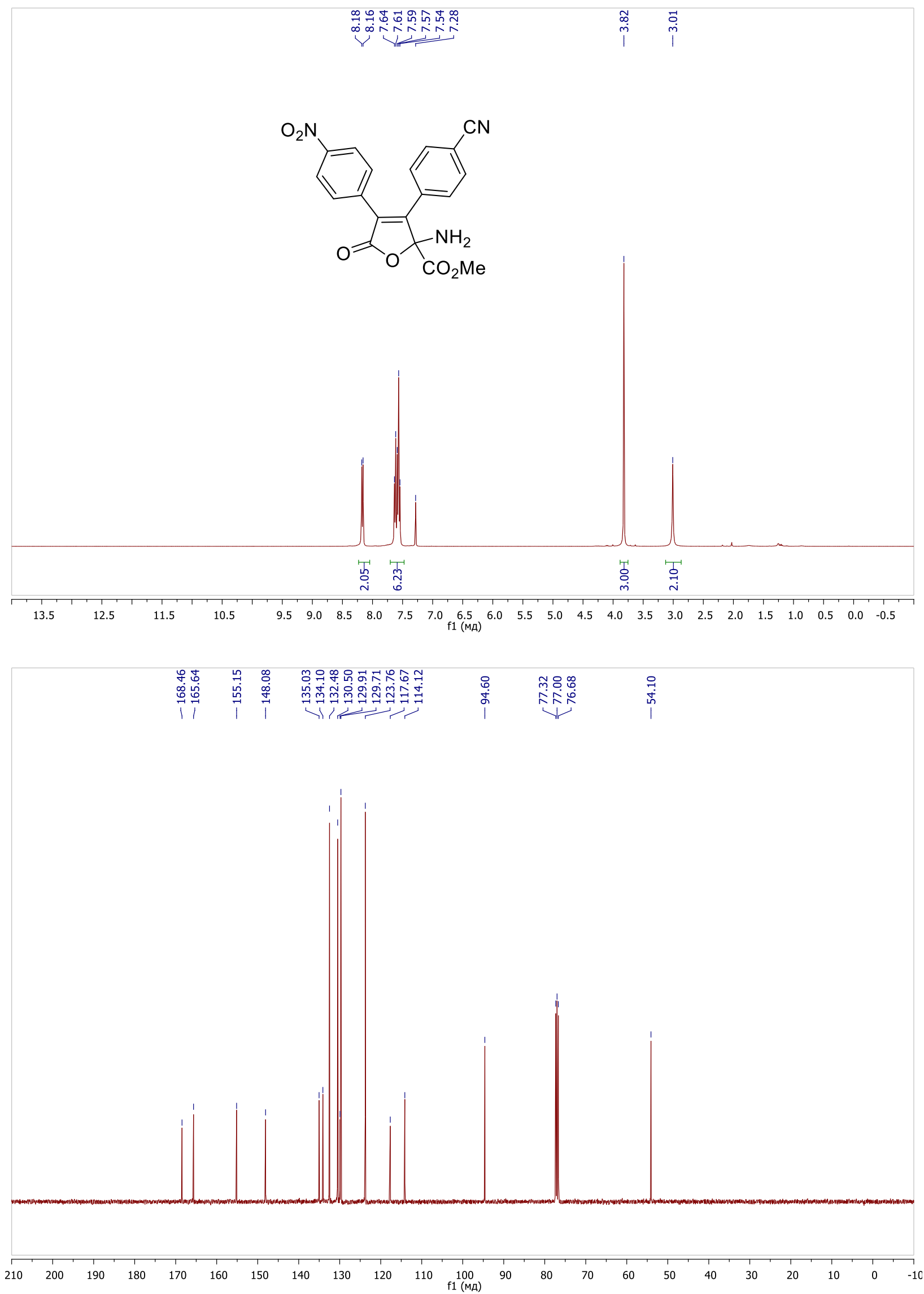
${ }^{1} \mathrm{H}\left(400 \mathrm{MHz}, \mathrm{CDCl}_{3}\right)$ and ${ }^{13} \mathrm{C}\left(100 \mathrm{MHz}, \mathrm{CDCl}_{3}\right)$ NMR spectra of compound $4 \mathbf{l}$
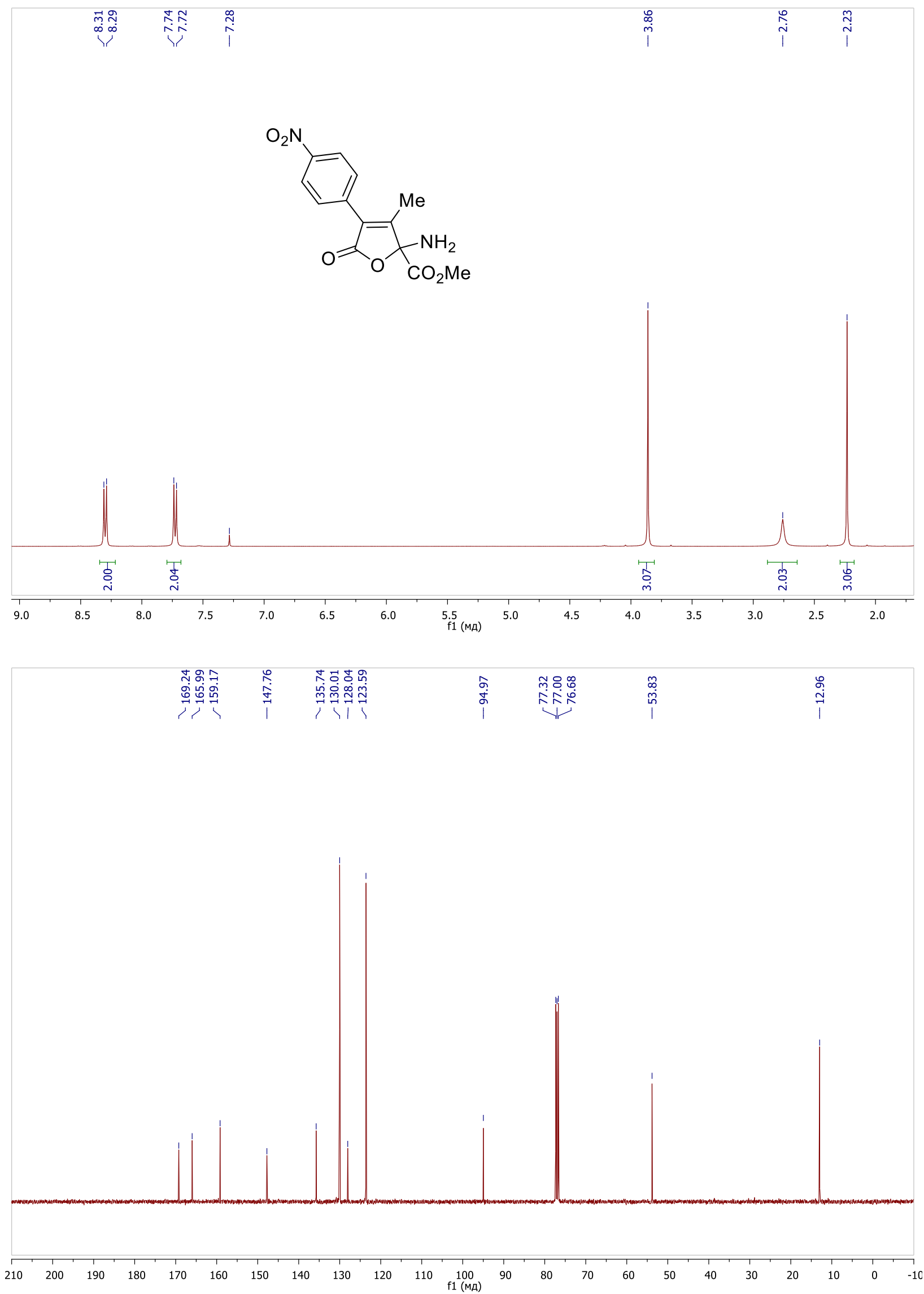
HMBC ${ }^{1} \mathrm{H}^{13}{ }^{13} \mathrm{C}$ and NOESY NMR spectra $\left(\mathrm{CDCl}_{3}\right)$ of compound $4 \mathbf{l}$
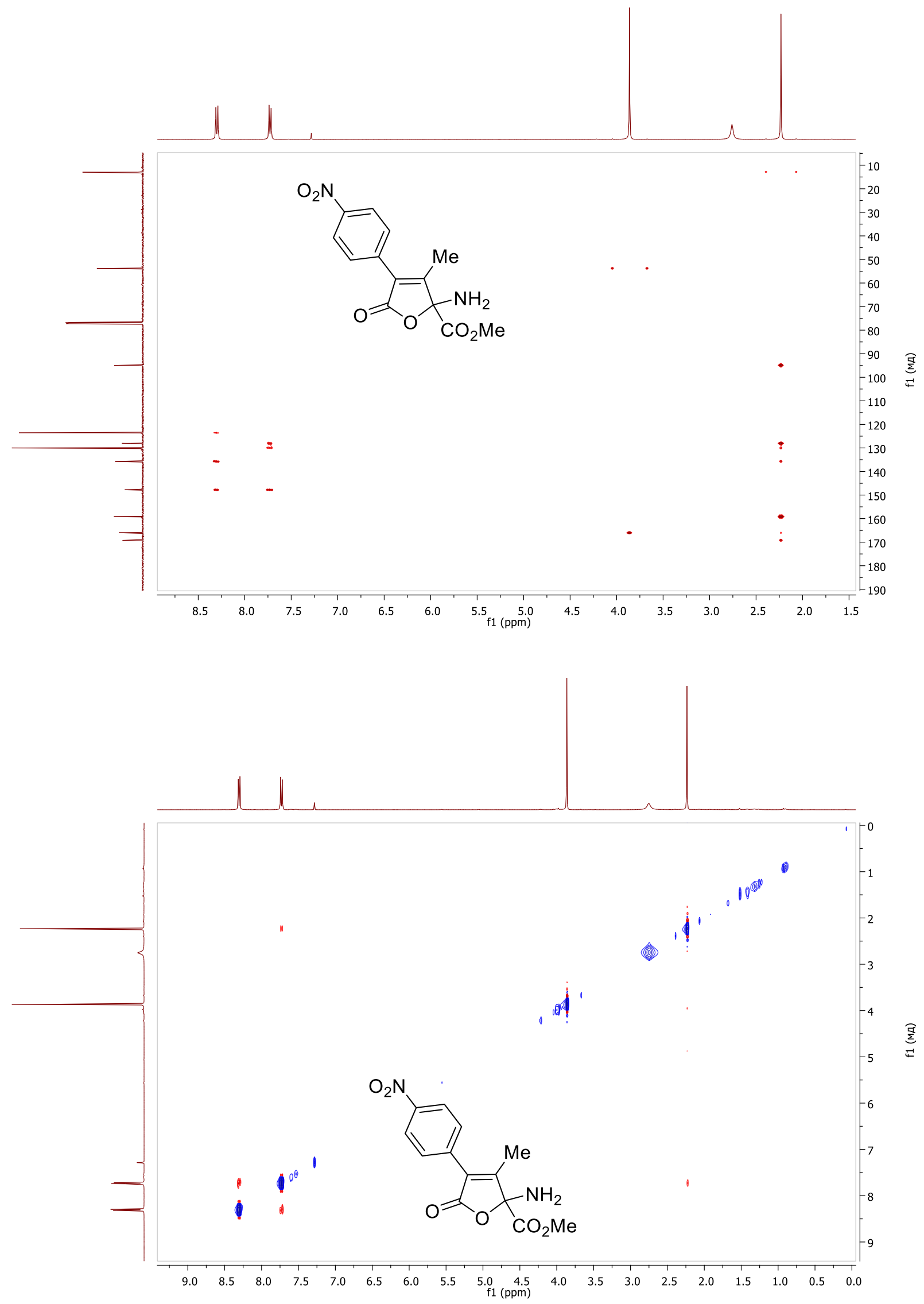
${ }^{1} \mathrm{H}\left(400 \mathrm{MHz}, \mathrm{CDCl}_{3}\right)$ and ${ }^{13} \mathrm{C}\left(100 \mathrm{MHz}, \mathrm{CDCl}_{3}\right)$ NMR spectra of compound $\mathbf{4 m}$
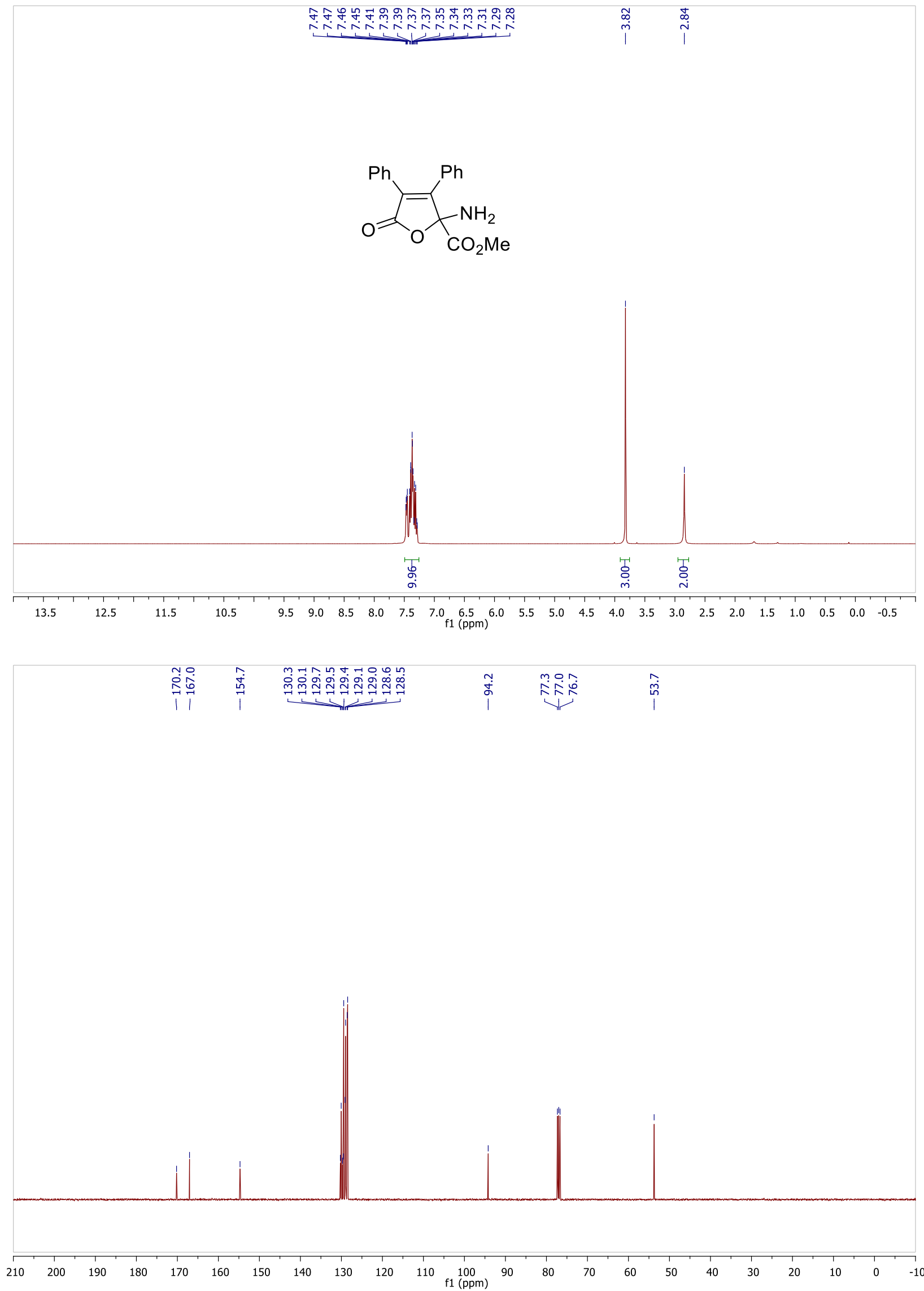
${ }^{1} \mathrm{H}\left(400 \mathrm{MHz}, \mathrm{CDCl}_{3}\right)$ and ${ }^{13} \mathrm{C}\left(100 \mathrm{MHz}, \mathrm{CDCl}_{3}\right) \mathrm{NMR}$ spectra of compound $\mathbf{4 n}$
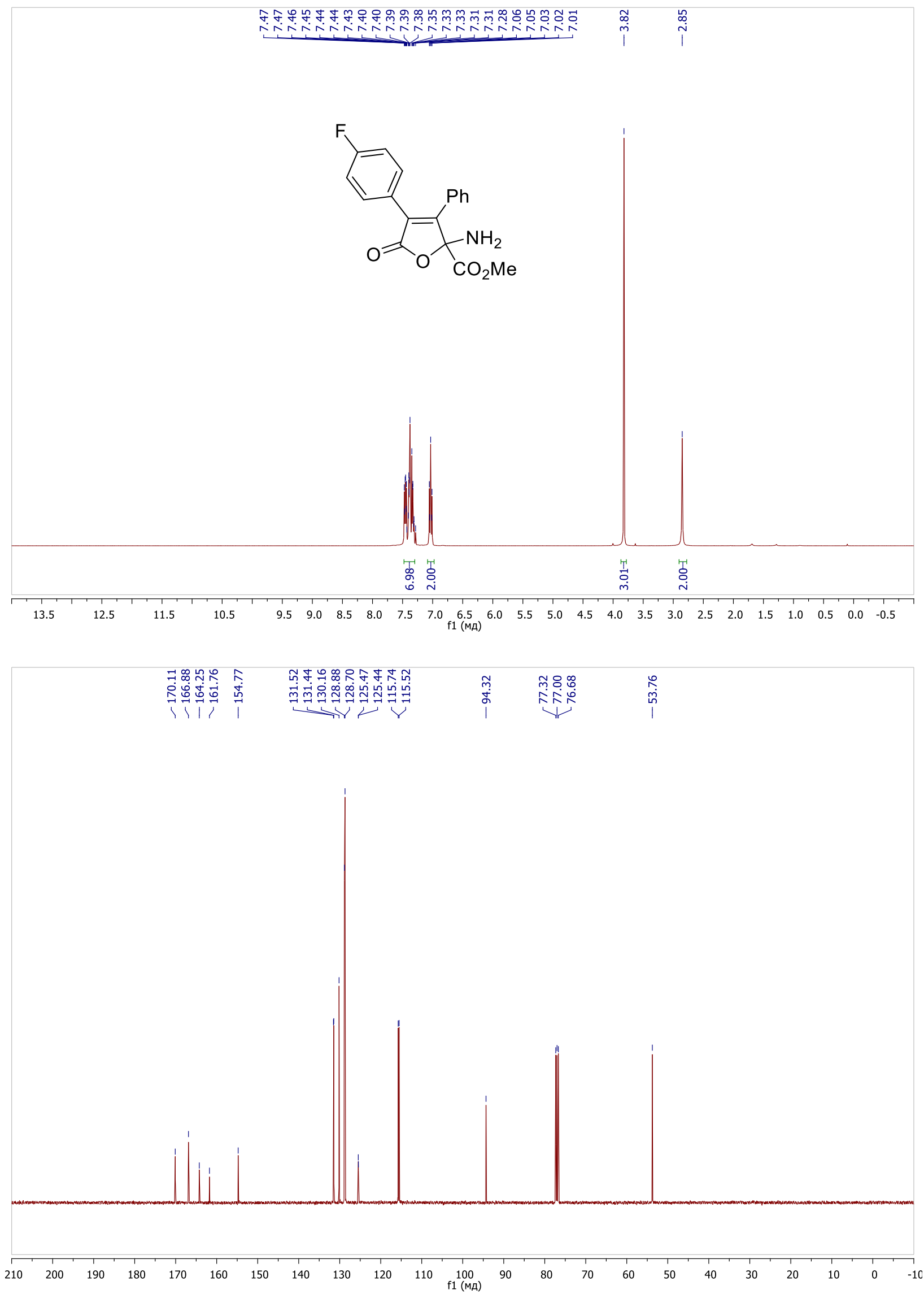
${ }^{1} \mathrm{H}\left(400 \mathrm{MHz}, \mathrm{CDCl}_{3}\right)$ and ${ }^{13} \mathrm{C}\left(100 \mathrm{MHz}, \mathrm{CDCl}_{3}\right)$ NMR spectra of compound $4 \mathrm{o}$
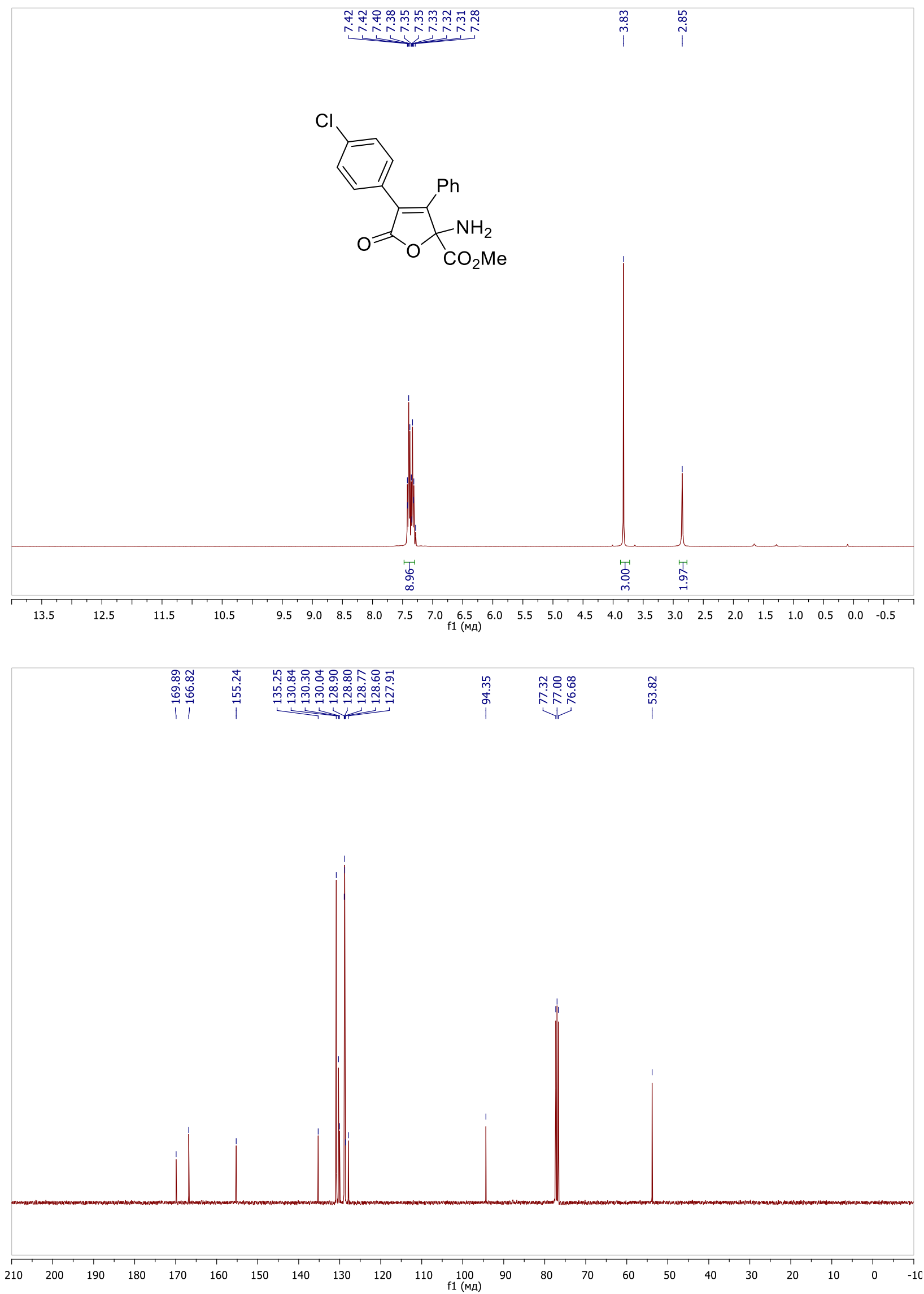
${ }^{1} \mathrm{H}\left(400 \mathrm{MHz}, \mathrm{CDCl}_{3}\right)$ and ${ }^{13} \mathrm{C}\left(100 \mathrm{MHz}, \mathrm{CDCl}_{3}\right) \mathrm{NMR}$ spectra of compound $\mathbf{4 p}$
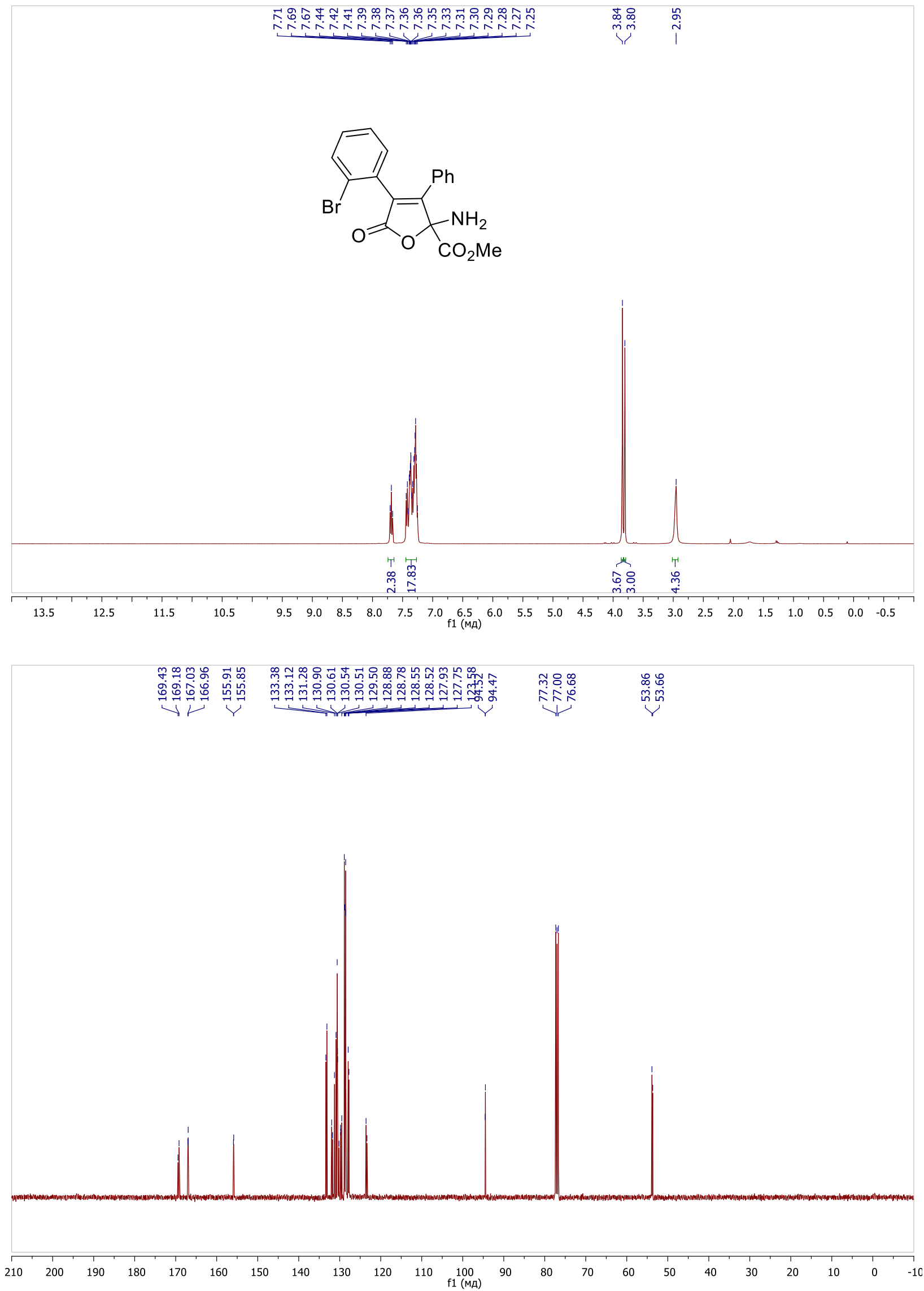
HMBC ${ }^{1} \mathrm{H}^{13} \mathrm{C}^{1} \mathrm{NMR}$ spectrum $\left(\mathrm{CDCl}_{3}\right)$ of compound $\mathbf{4 p}$

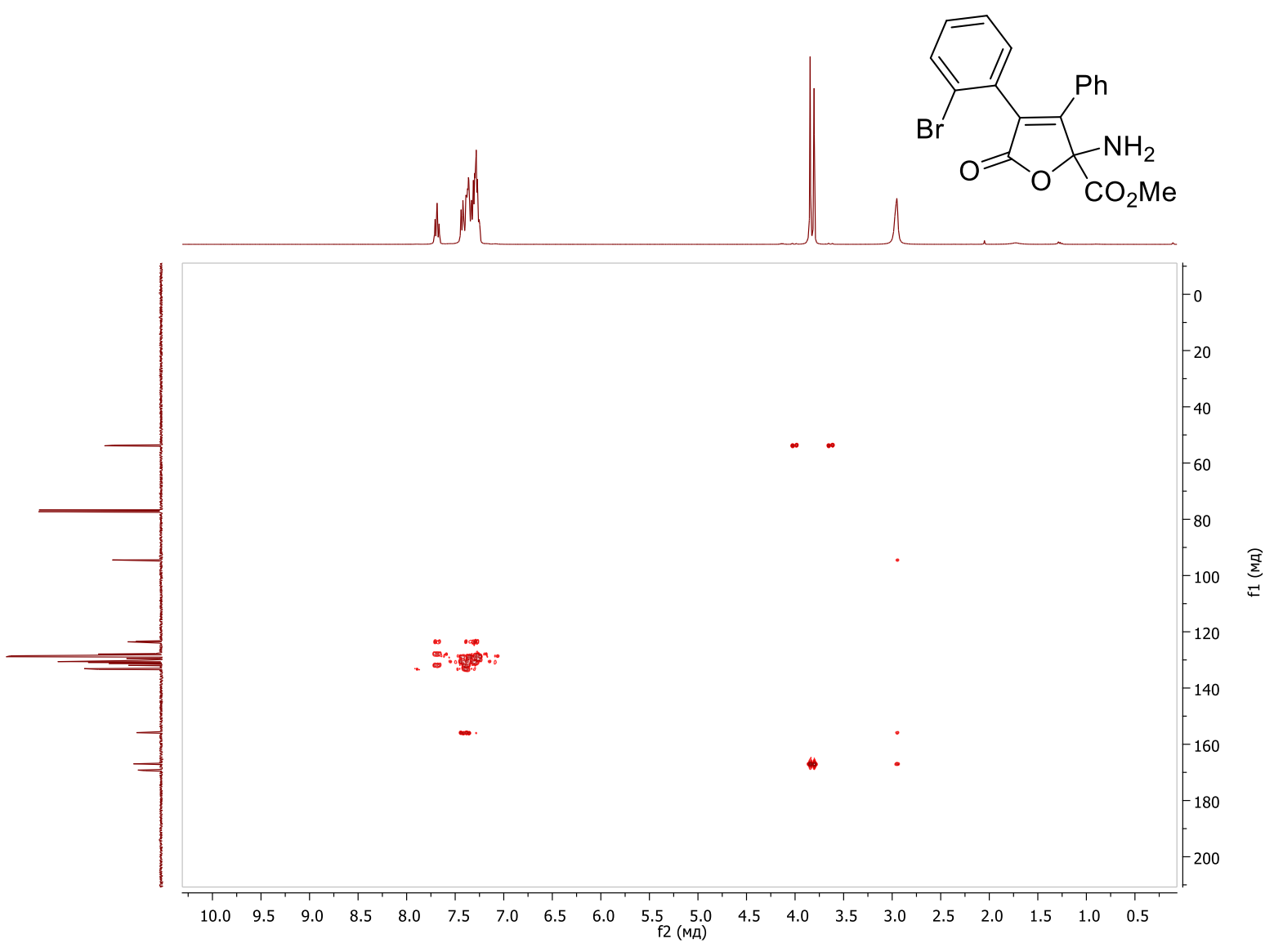


${ }^{1} \mathrm{H}\left(400 \mathrm{MHz}, \mathrm{CDCl}_{3}\right)$ and ${ }^{13} \mathrm{C}\left(100 \mathrm{MHz}, \mathrm{CDCl}_{3}\right) \mathrm{NMR}$ spectra of compound $\mathbf{4 q}$
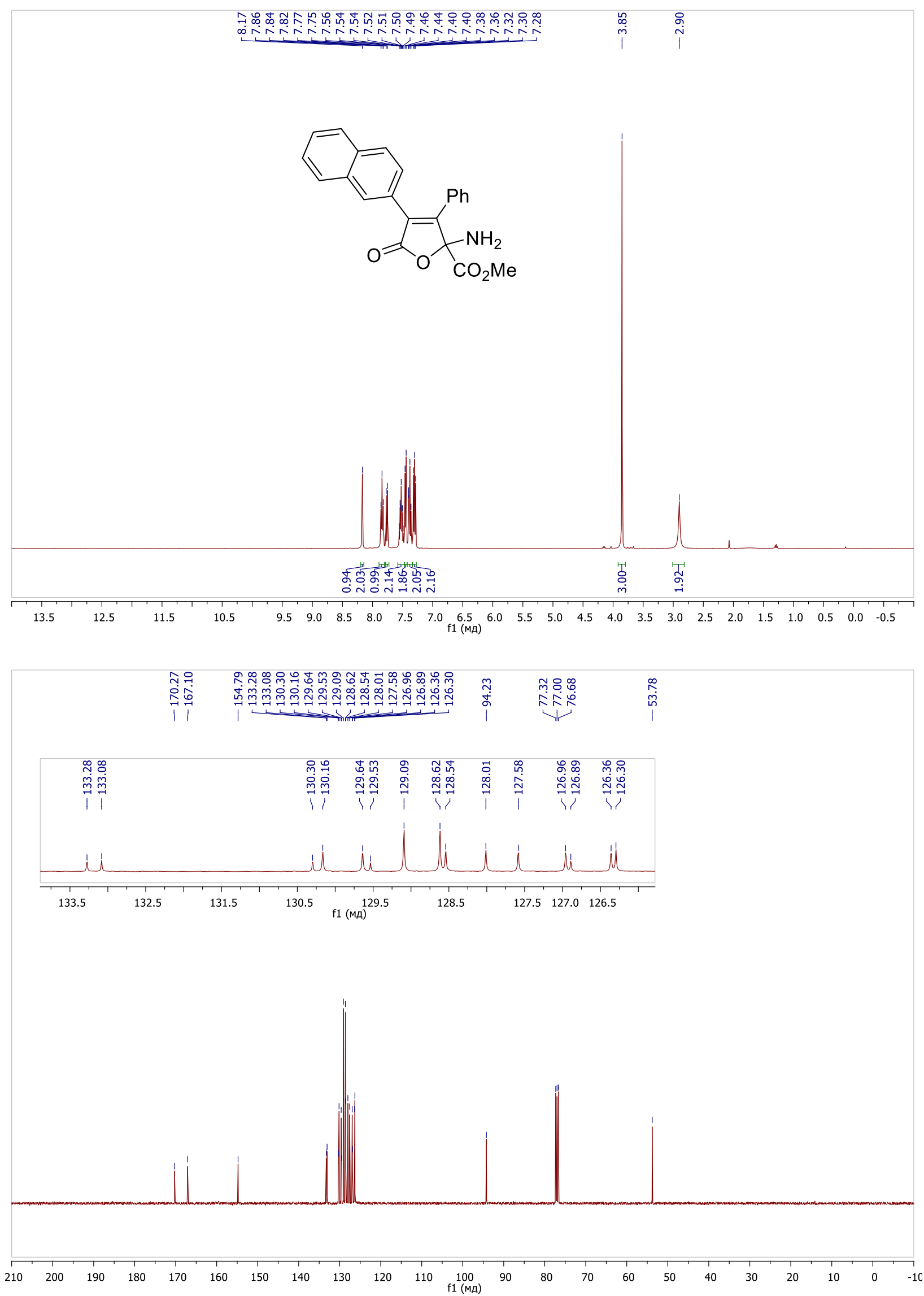
HMBC ${ }^{1} \mathrm{H}^{-13} \mathrm{C}$ NMR spectrum $\left(\mathrm{CDCl}_{3}\right)$ of compound $\mathbf{4 q}$

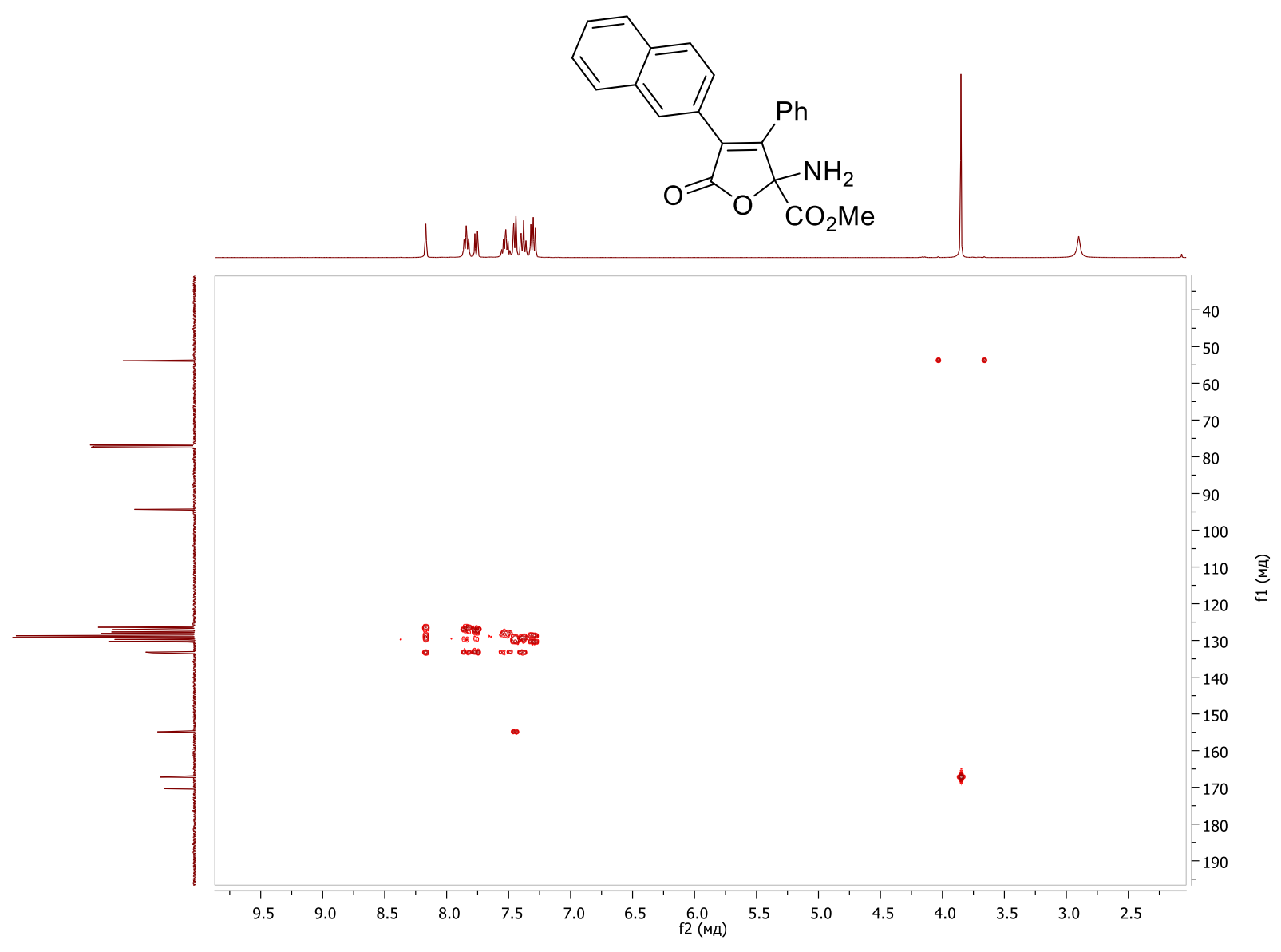


${ }^{1} \mathrm{H}\left(400 \mathrm{MHz}, \mathrm{CDCl}_{3}\right)$ and ${ }^{13} \mathrm{C}\left(100 \mathrm{MHz}, \mathrm{CDCl}_{3}\right)$ NMR spectra of compound $4 \mathbf{r}$
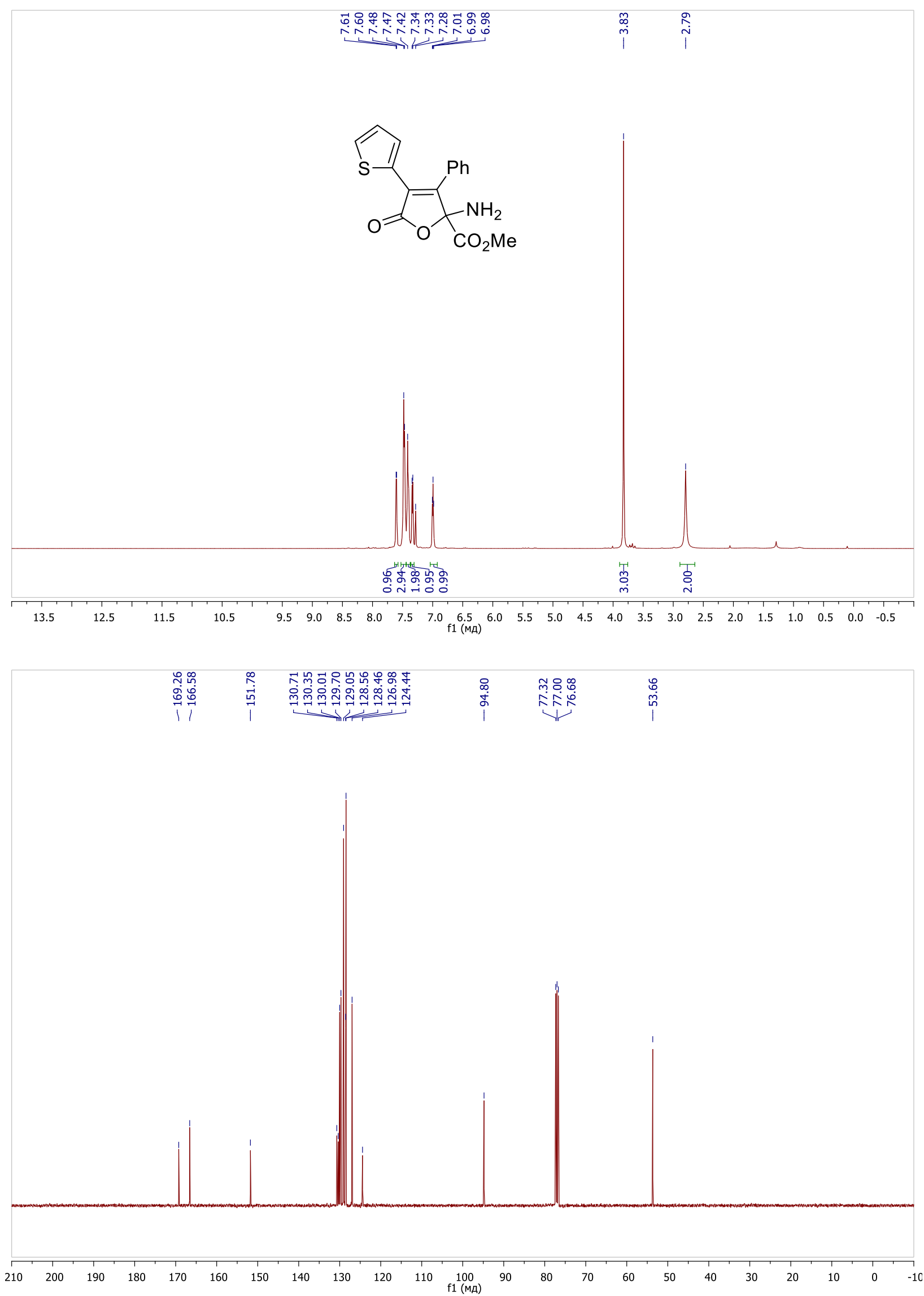
HMBC ${ }^{1} \mathrm{H}^{13} \mathrm{C}^{13} \mathrm{NR}$ spectrum $\left(\mathrm{CDCl}_{3}\right)$ of compound $\mathbf{4 r}$

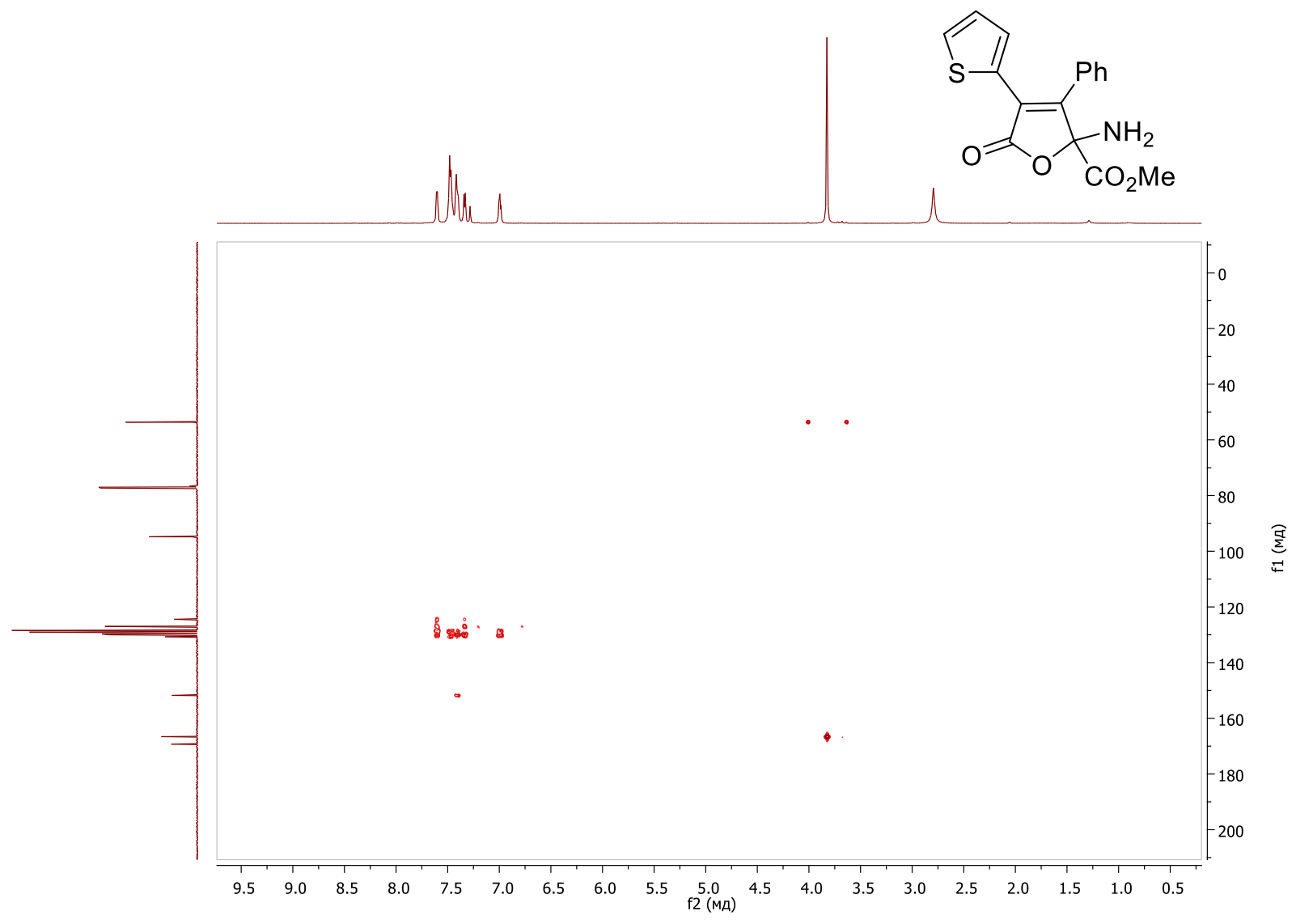


${ }^{1} \mathrm{H}\left(400 \mathrm{MHz}, \mathrm{CDCl}_{3}\right)$ and ${ }^{13} \mathrm{C}\left(100 \mathrm{MHz}, \mathrm{CDCl}_{3}\right)$ NMR spectra of compound $4 \mathbf{s}$
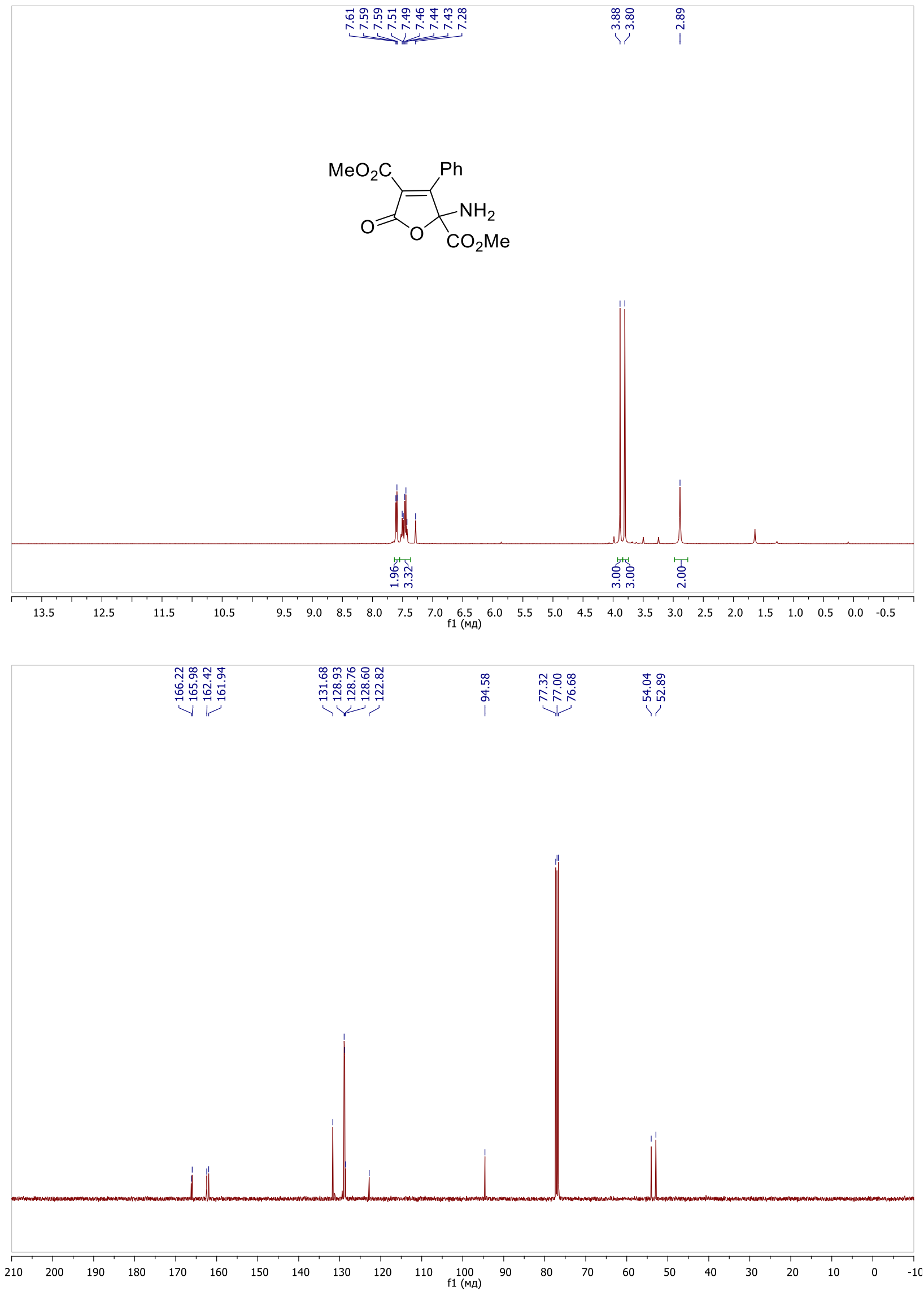
HMBC ${ }^{1} \mathrm{H}^{13}{ }^{13} \mathrm{C}$ NMR spectrum $\left(\mathrm{CDCl}_{3}\right)$ of compound $4 \mathrm{~s}$

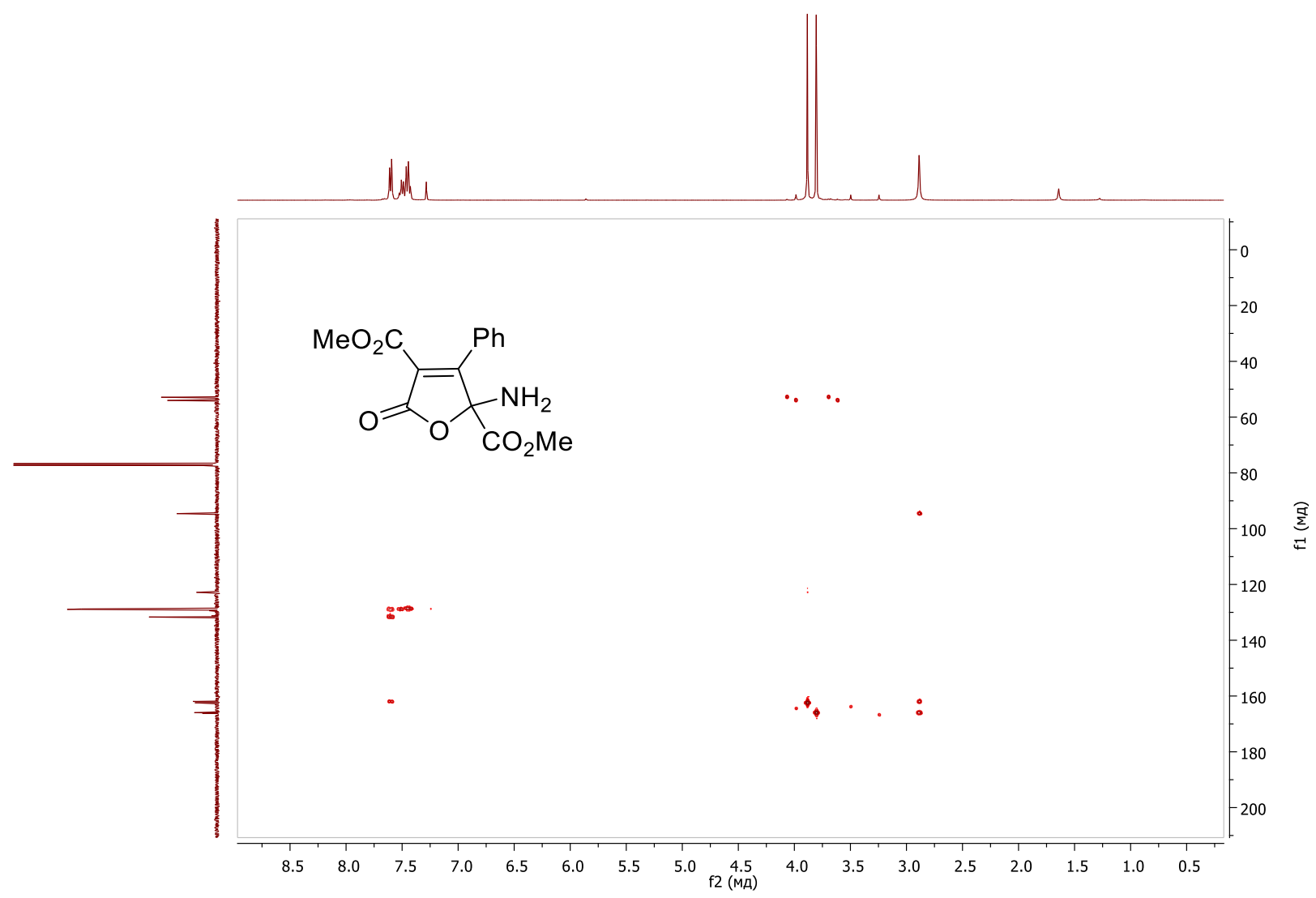


${ }^{1} \mathrm{H}\left(400 \mathrm{MHz}, \mathrm{CDCl}_{3}\right)$ and ${ }^{13} \mathrm{C}\left(100 \mathrm{MHz}, \mathrm{CDCl}_{3}\right)$ NMR spectra of compound $\mathbf{4 t}$

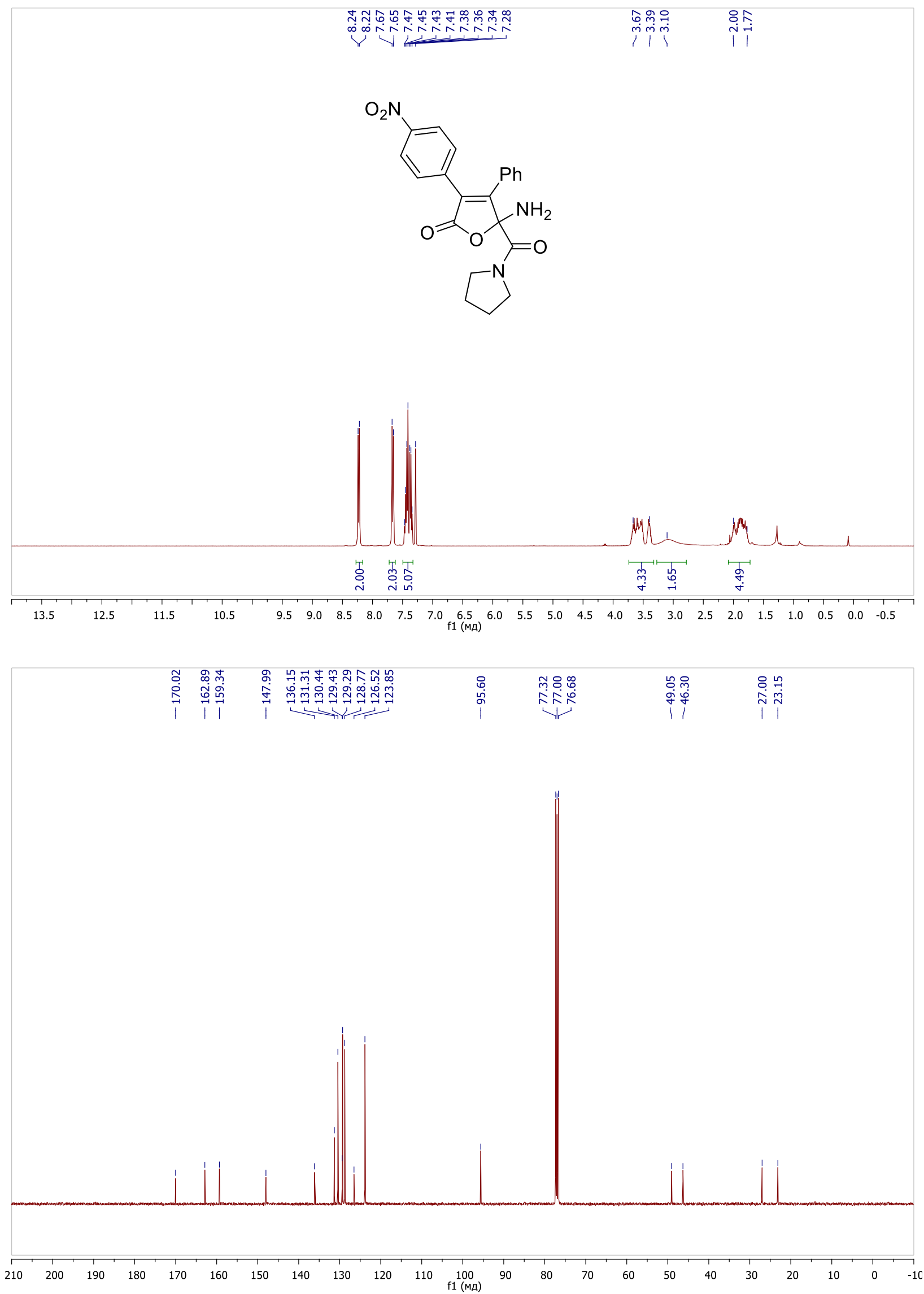


HMBC ${ }^{1} \mathrm{H}^{-13} \mathrm{C}$ NMR spectrum $\left(\mathrm{CDCl}_{3}\right)$ of compound $\mathbf{4 t}$

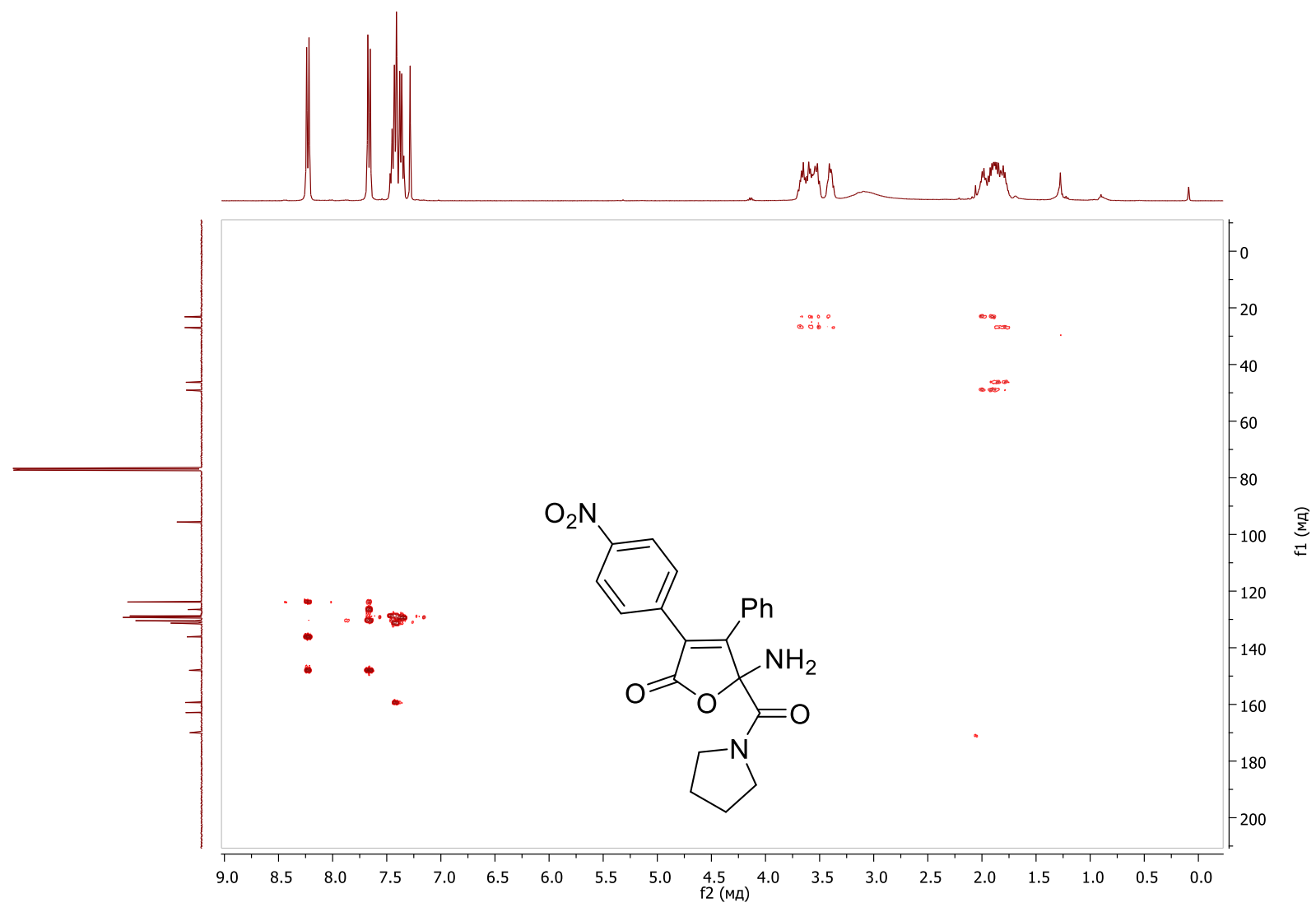


${ }^{1} \mathrm{H}\left(400 \mathrm{MHz}, \mathrm{CDCl}_{3}\right)$ and ${ }^{13} \mathrm{C}\left(100 \mathrm{MHz}, \mathrm{CDCl}_{3}\right)$ NMR spectra of compound $\mathbf{4} \mathbf{u}$
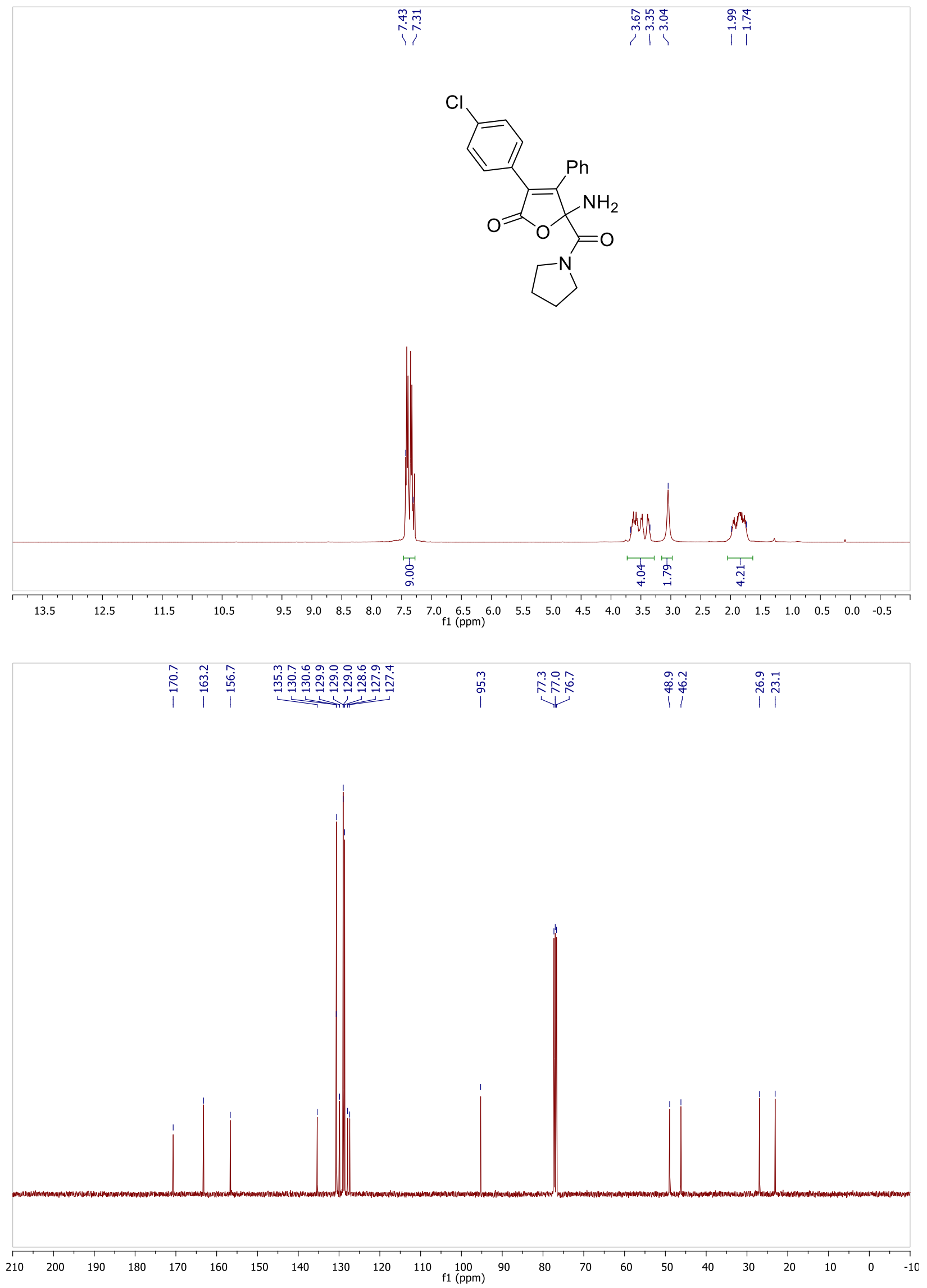
HMBC ${ }^{1} \mathrm{H}^{13}{ }^{13}$ NMR spectrum $\left(\mathrm{CDCl}_{3}\right)$ of compound $4 \mathbf{u}$

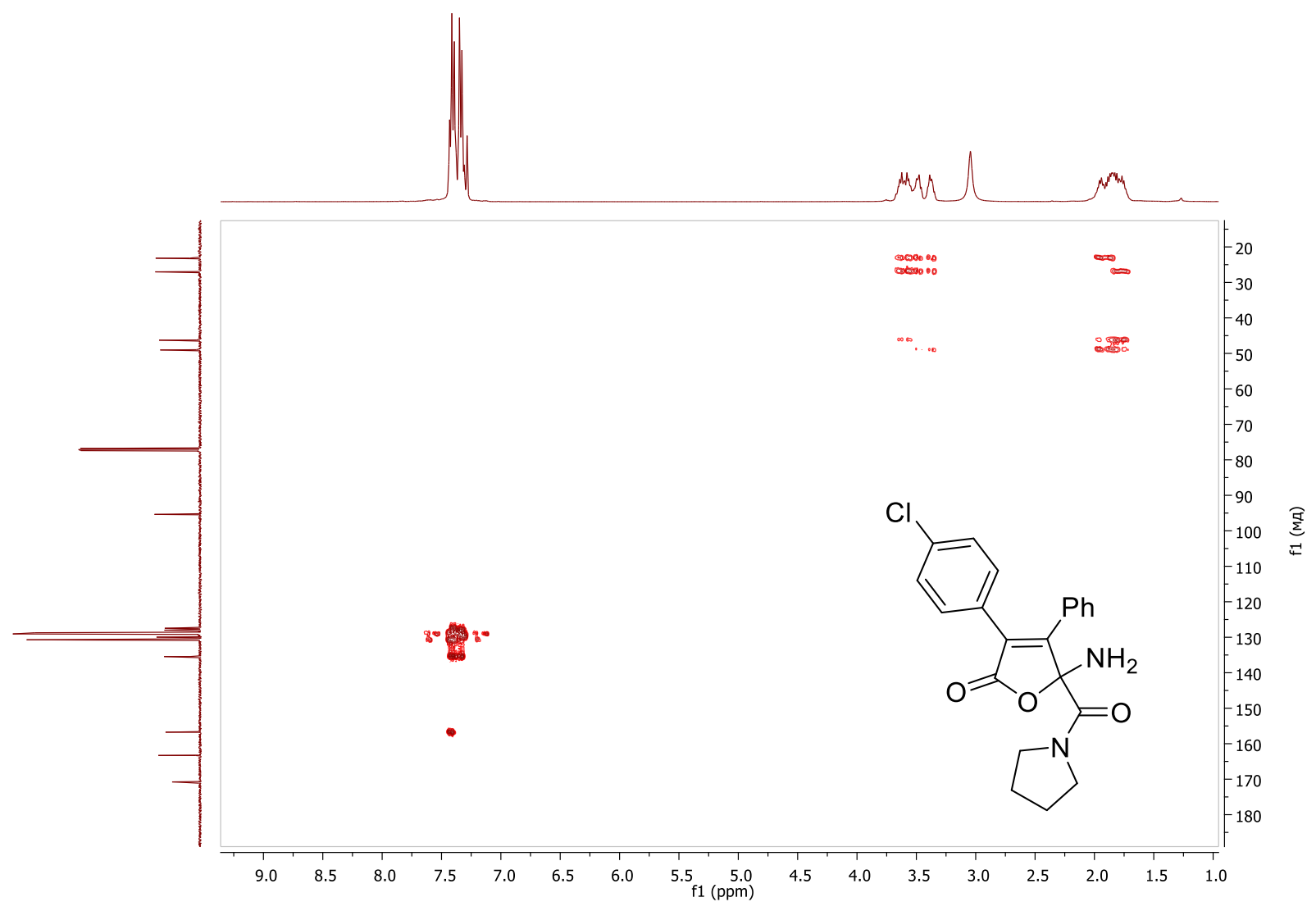


${ }^{1} \mathrm{H}\left(400 \mathrm{MHz}, \mathrm{CDCl}_{3}\right)$ and ${ }^{13} \mathrm{C}\left(100 \mathrm{MHz}, \mathrm{CDCl}_{3}\right)$ NMR spectra of compounds $4 \mathbf{w}$ and $\mathbf{5 n}$
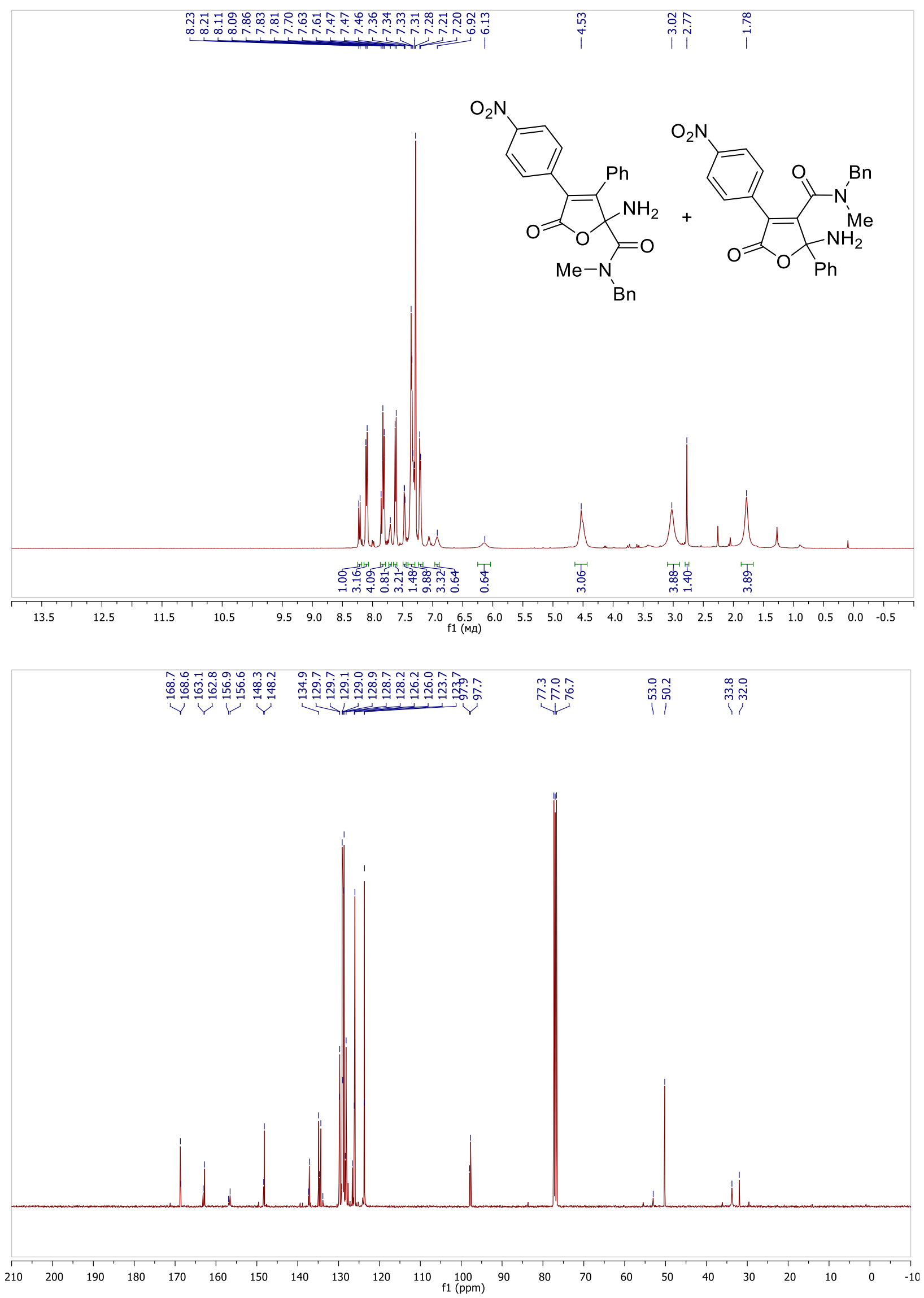
HMBC ${ }^{1} \mathrm{H}_{-}{ }^{13} \mathrm{C}$ NMR spectrum $\left(\mathrm{CDCl}_{3}\right)$ of compounds $\mathbf{4 w}$ and $\mathbf{5 n}$

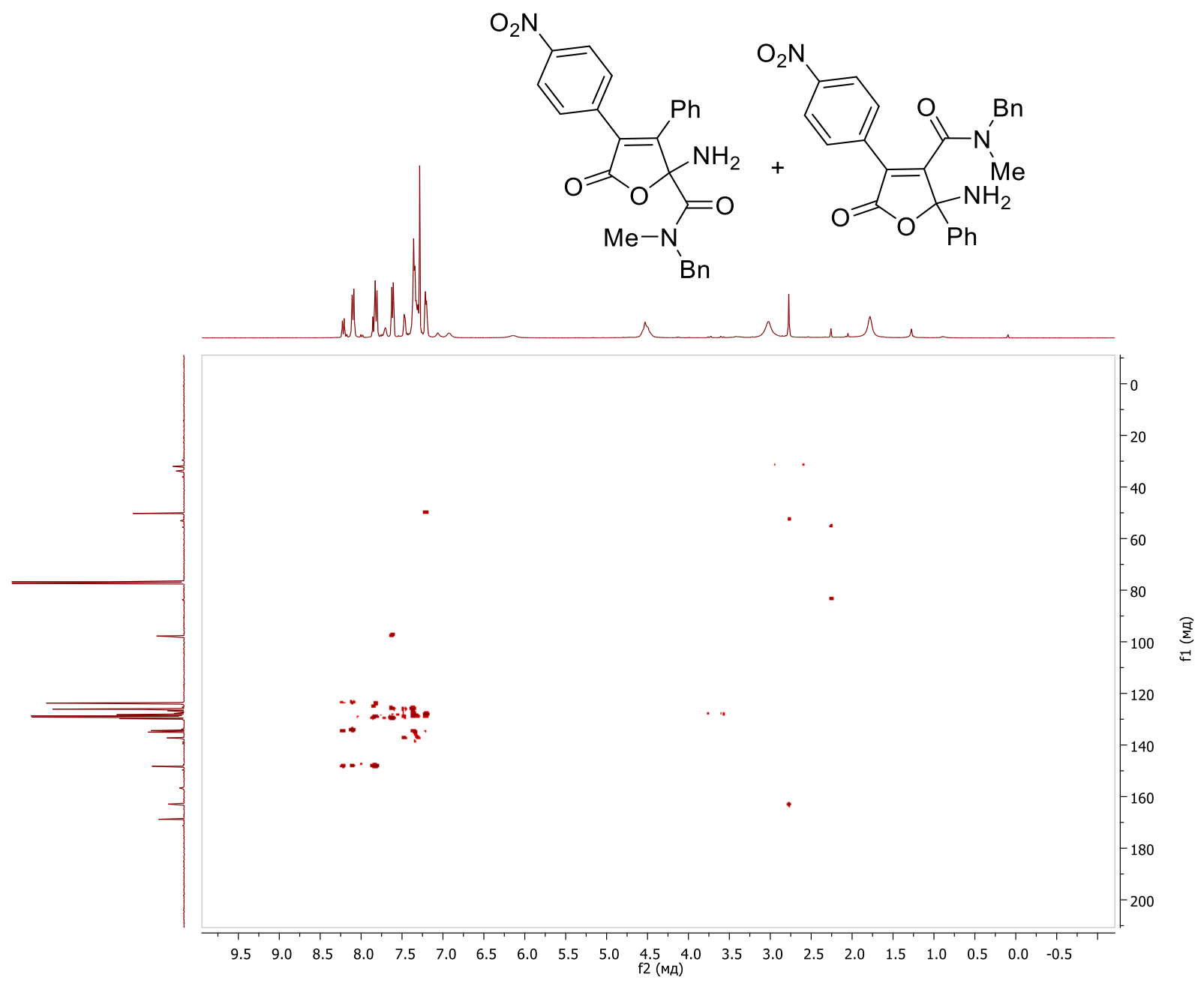


${ }^{1} \mathrm{H}\left(400 \mathrm{MHz}, \mathrm{CDCl}_{3}\right)$ and ${ }^{13} \mathrm{C}\left(100 \mathrm{MHz}, \mathrm{CDCl}_{3}\right)$ NMR spectra of compounds $\mathbf{5 o}$ and $\mathbf{4 x}$
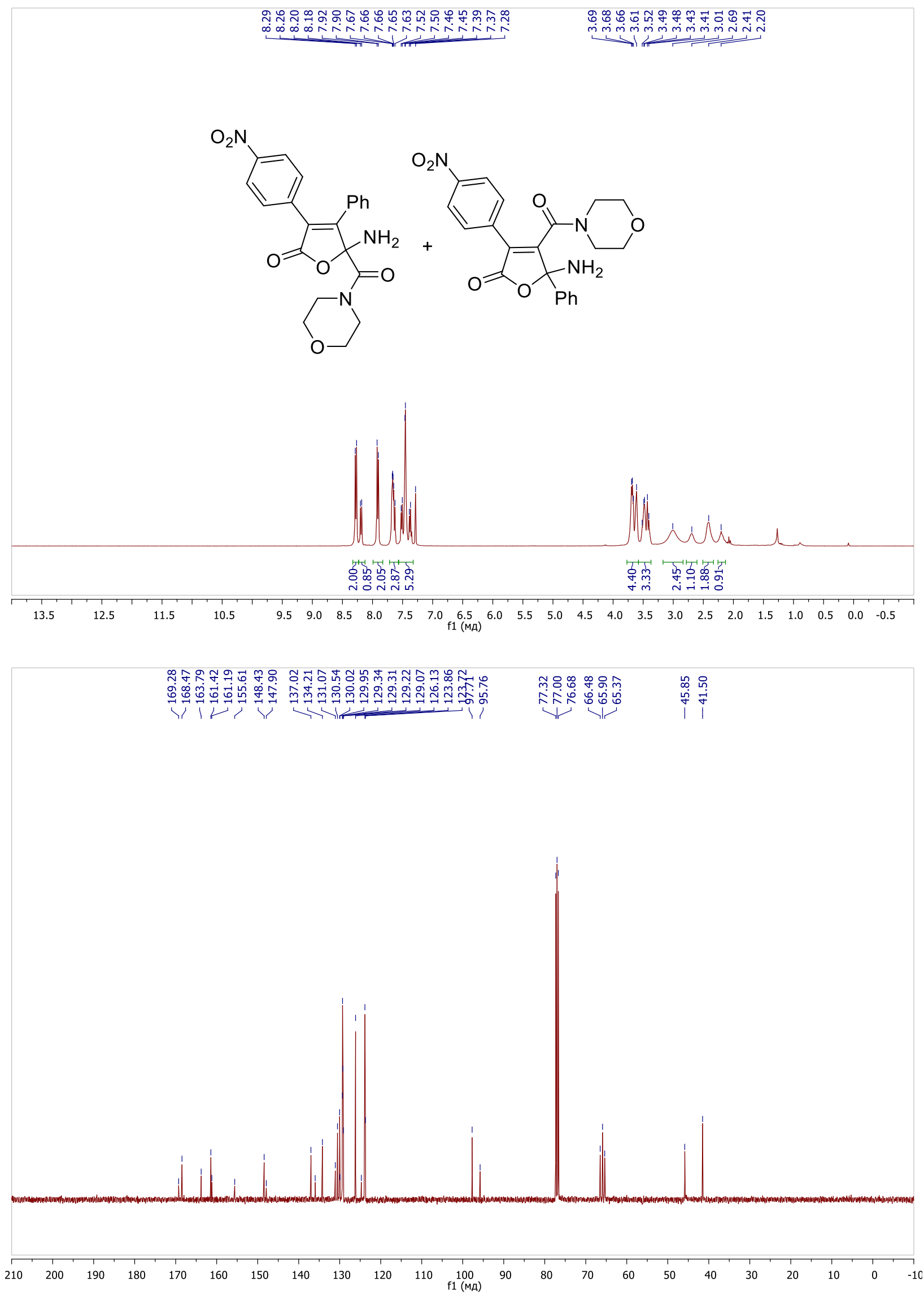
HMBC ${ }^{1} \mathrm{H}^{-13} \mathrm{C}$ NMR spectrum $\left(\mathrm{CDCl}_{3}\right)$ of compounds $\mathbf{5 o}$ and $\mathbf{4} \mathbf{x}$

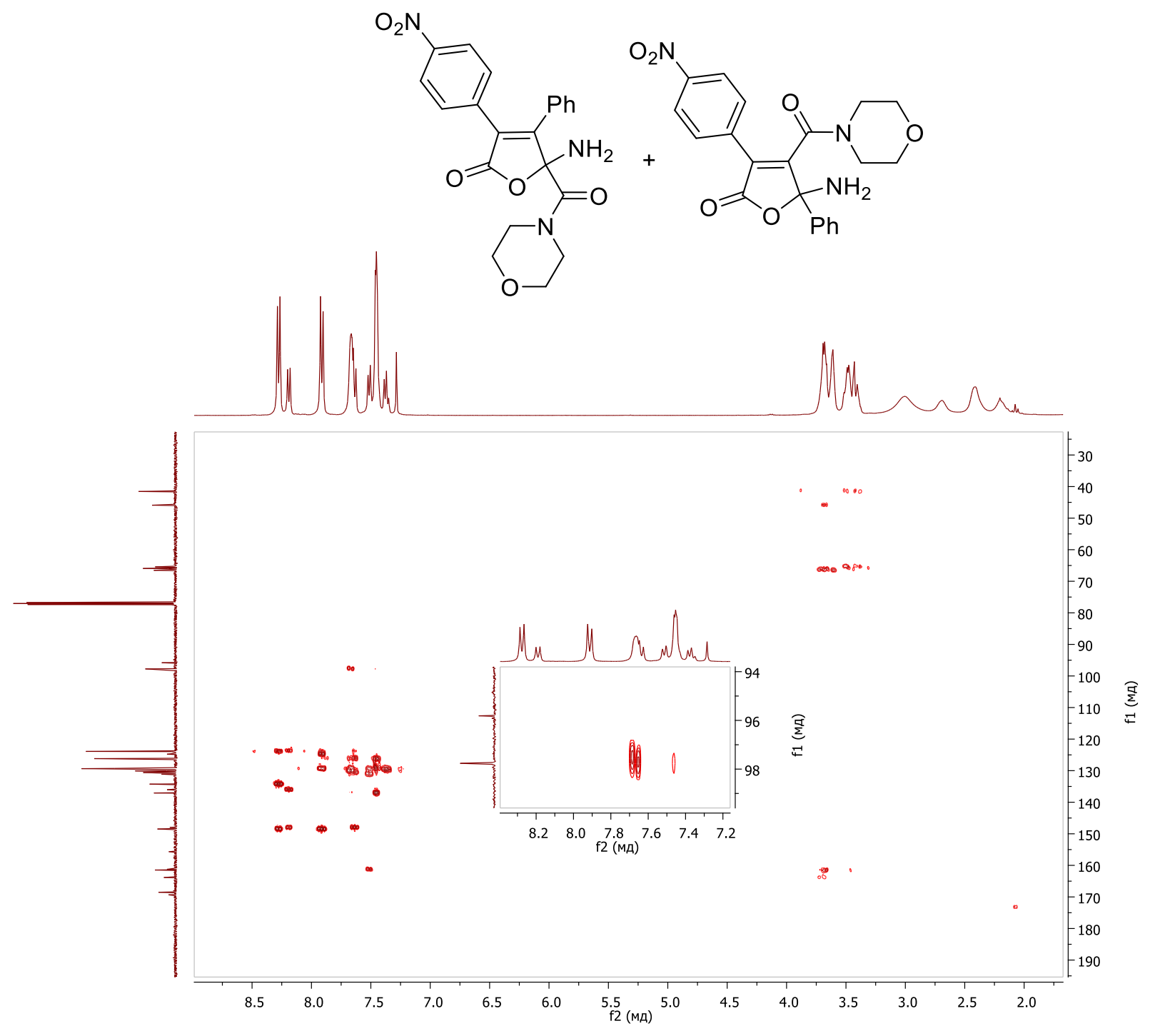


${ }^{1} \mathrm{H}\left(400 \mathrm{MHz}, \mathrm{CDCl}_{3}\right)$ and ${ }^{13} \mathrm{C}\left(100 \mathrm{MHz}, \mathrm{CDCl}_{3}\right)$ NMR spectra of compounds $4 \mathbf{y}$ and $\mathbf{5 p}$
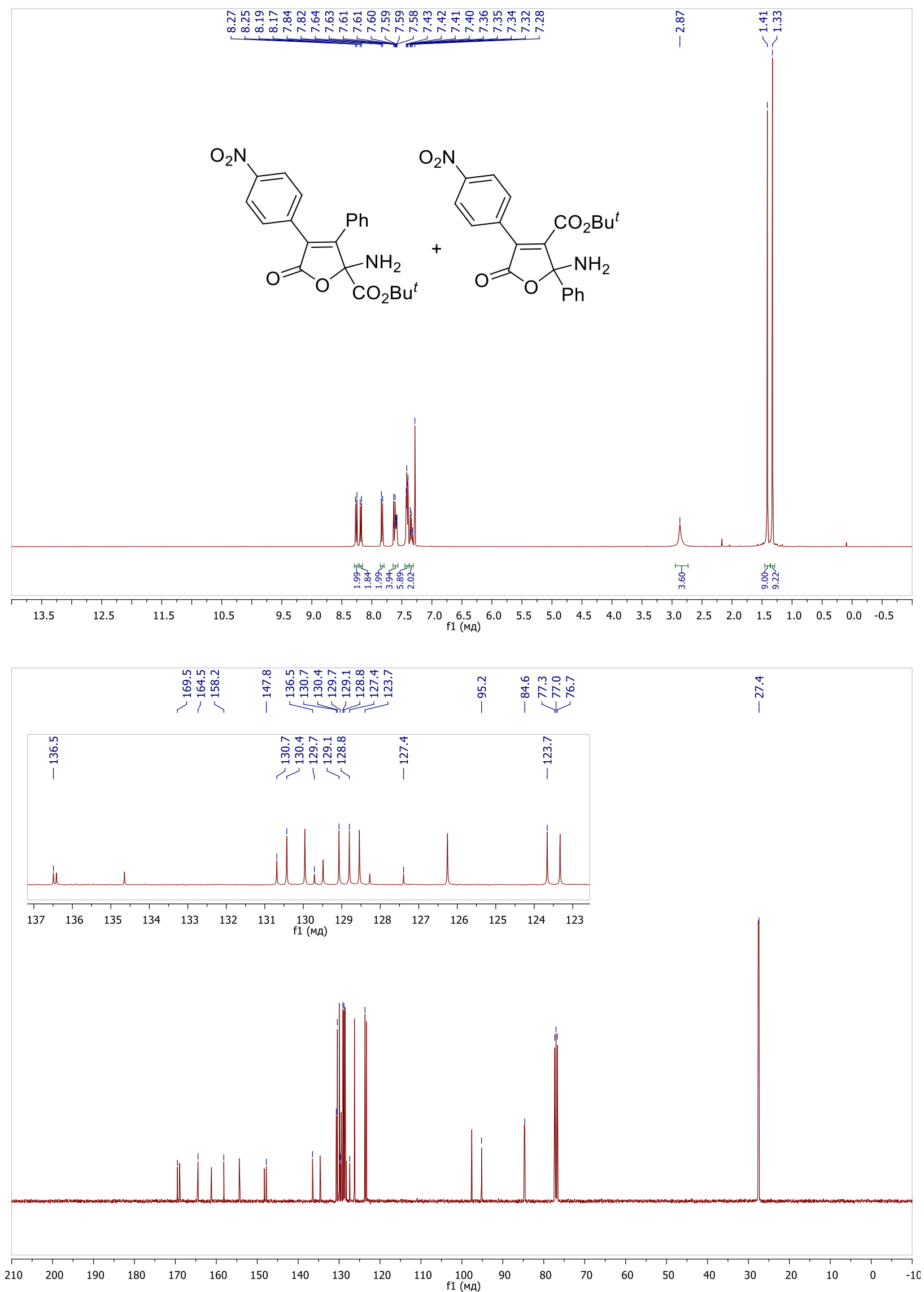
${ }^{1} \mathrm{H}\left(400 \mathrm{MHz}, \mathrm{CDCl}_{3}\right)$ and ${ }^{13} \mathrm{C}\left(100 \mathrm{MHz}, \mathrm{CDCl}_{3}\right)$ NMR spectra of compounds $4 \mathbf{y}$ and $\mathbf{5 p}$
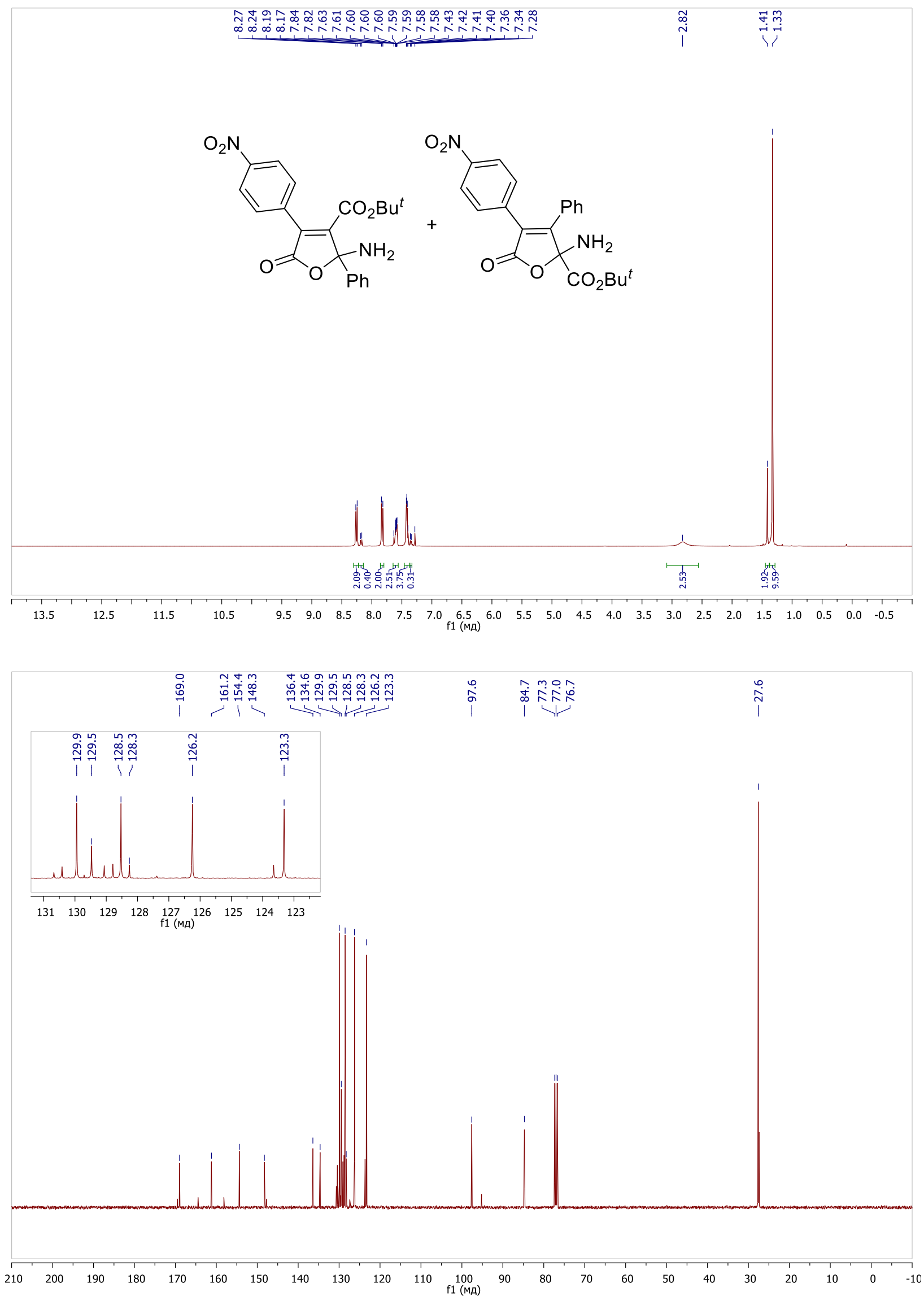
HMBC ${ }^{1} \mathrm{H}^{-13} \mathrm{C}$ NMR spectrum $\left(\mathrm{CDCl}_{3}\right)$ of compounds $4 \mathbf{y}$ and $\mathbf{5 p}$
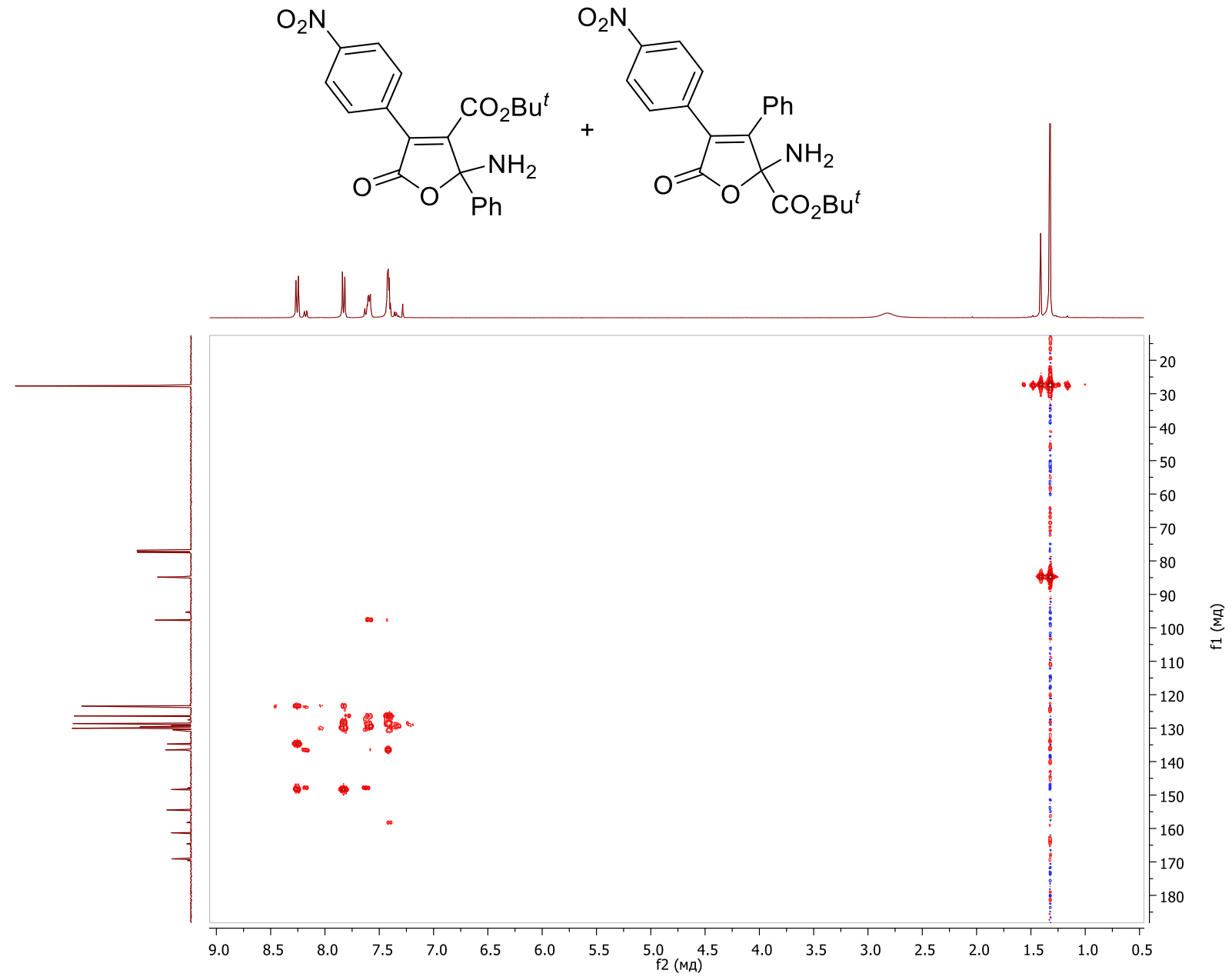
${ }^{1} \mathrm{H}\left(400 \mathrm{MHz}, \mathrm{CDCl}_{3}\right)$ and ${ }^{13} \mathrm{C}\left(100 \mathrm{MHz}, \mathrm{CDCl}_{3}\right) \mathrm{NMR}$ spectra of compound $\mathbf{5 a}$

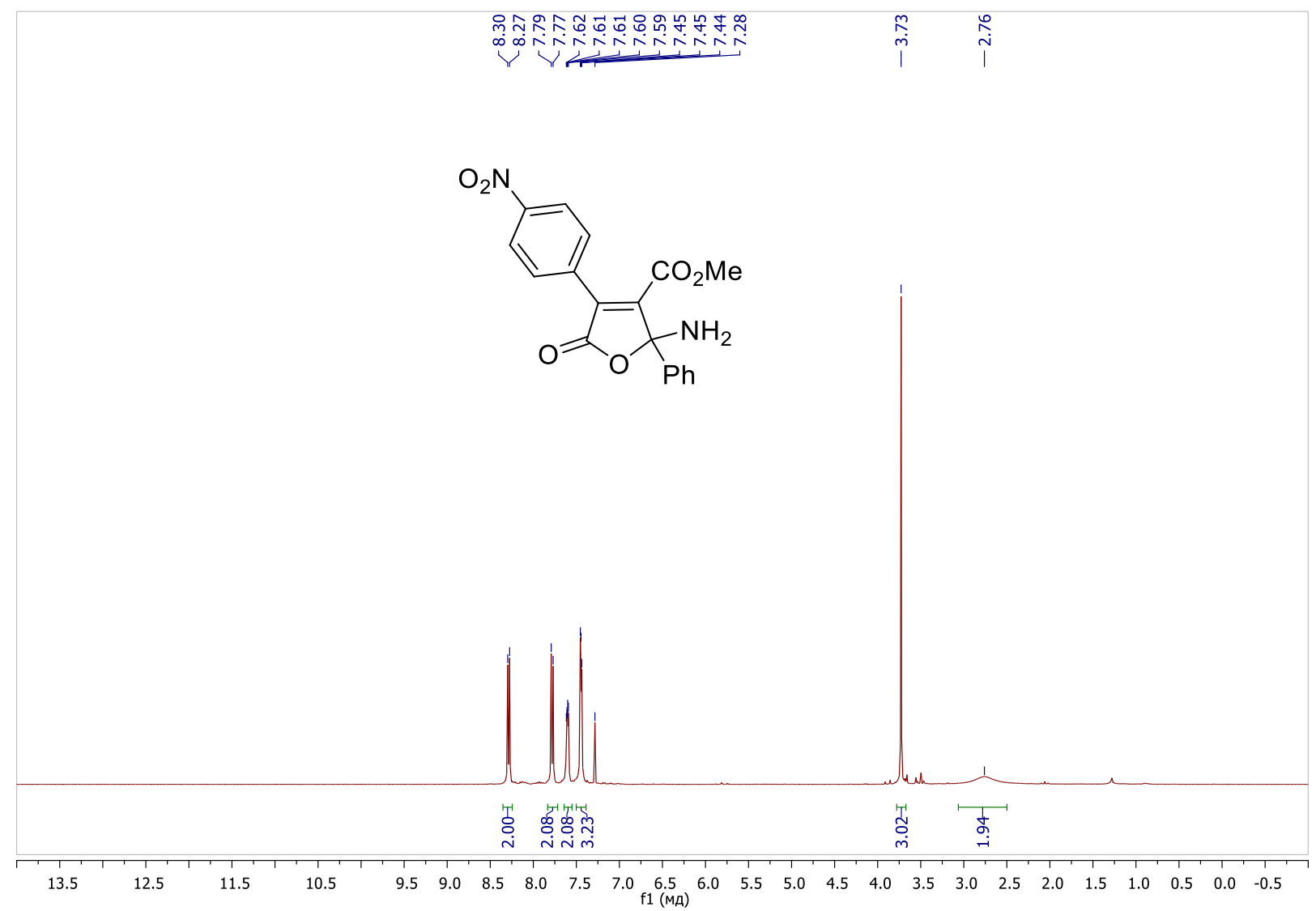

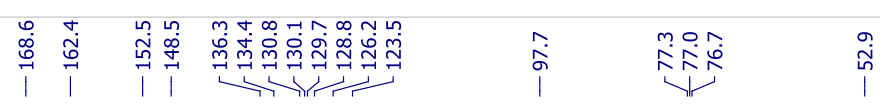

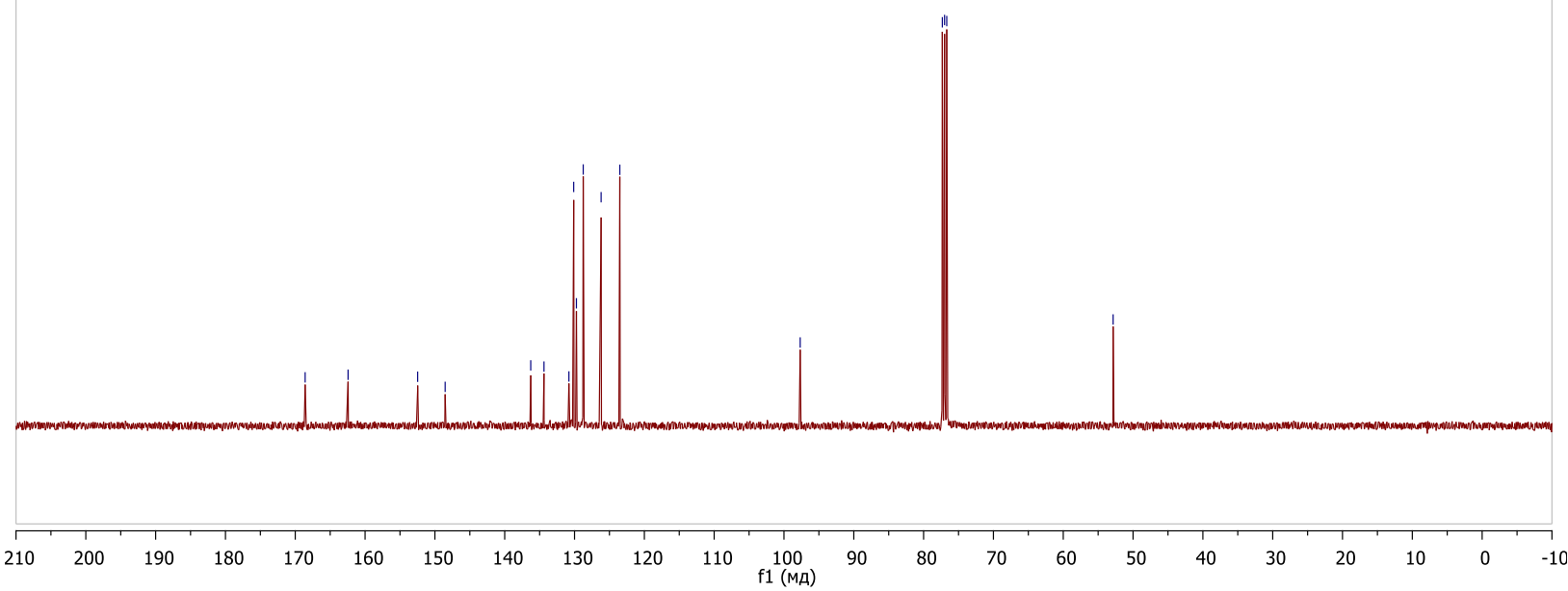


HSQC ${ }^{1} \mathrm{H}_{-}{ }^{13} \mathrm{C}$ and $\mathrm{HMBC}{ }^{1} \mathrm{H}^{13}{ }^{13} \mathrm{C}$ NMR spectra $\left(\mathrm{CDCl}_{3}\right)$ of compound $\mathbf{5 a}$
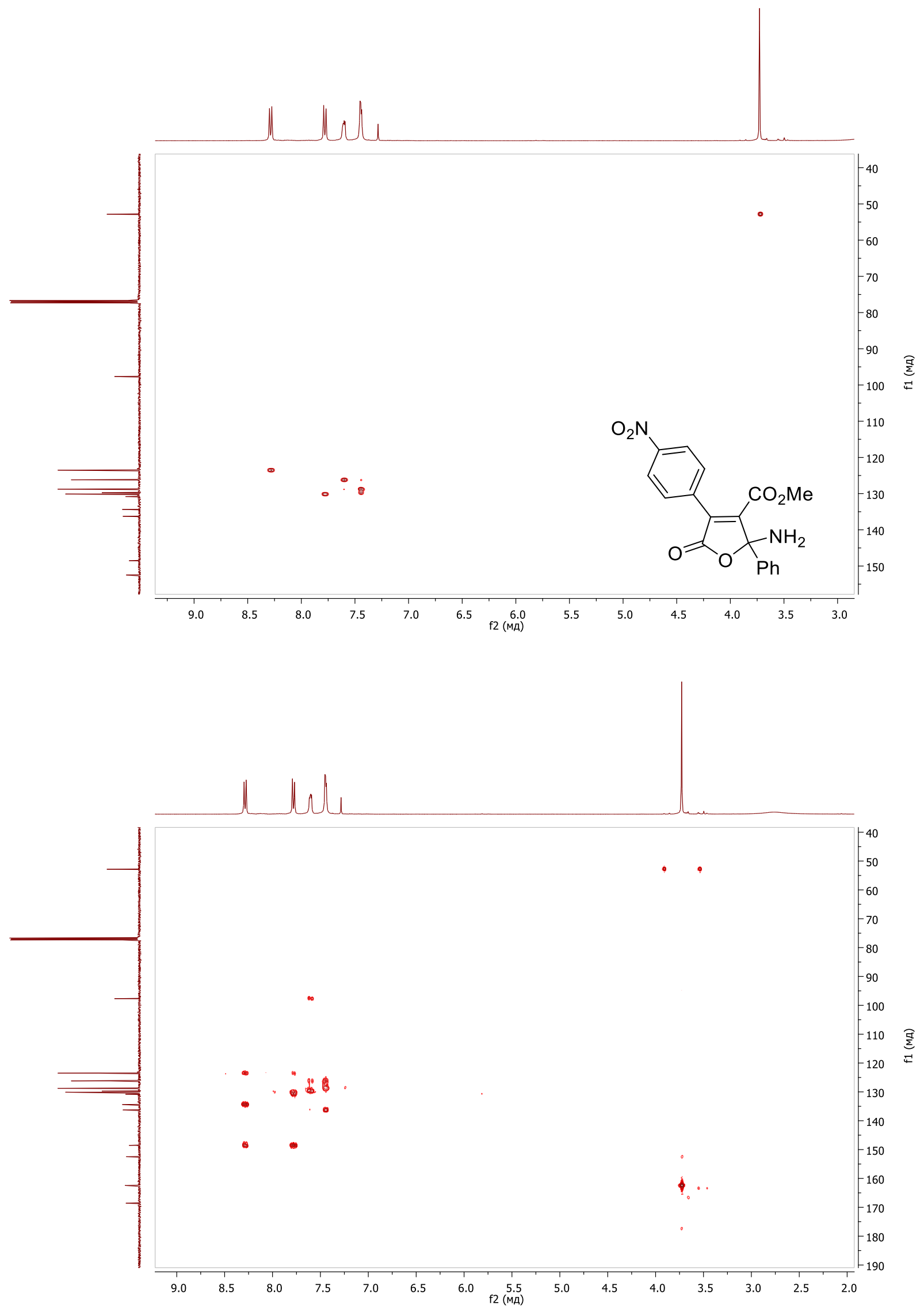
NOESY NMR spectrum $\left(\mathrm{CDCl}_{3}\right)$ of compound $\mathbf{5 a}$

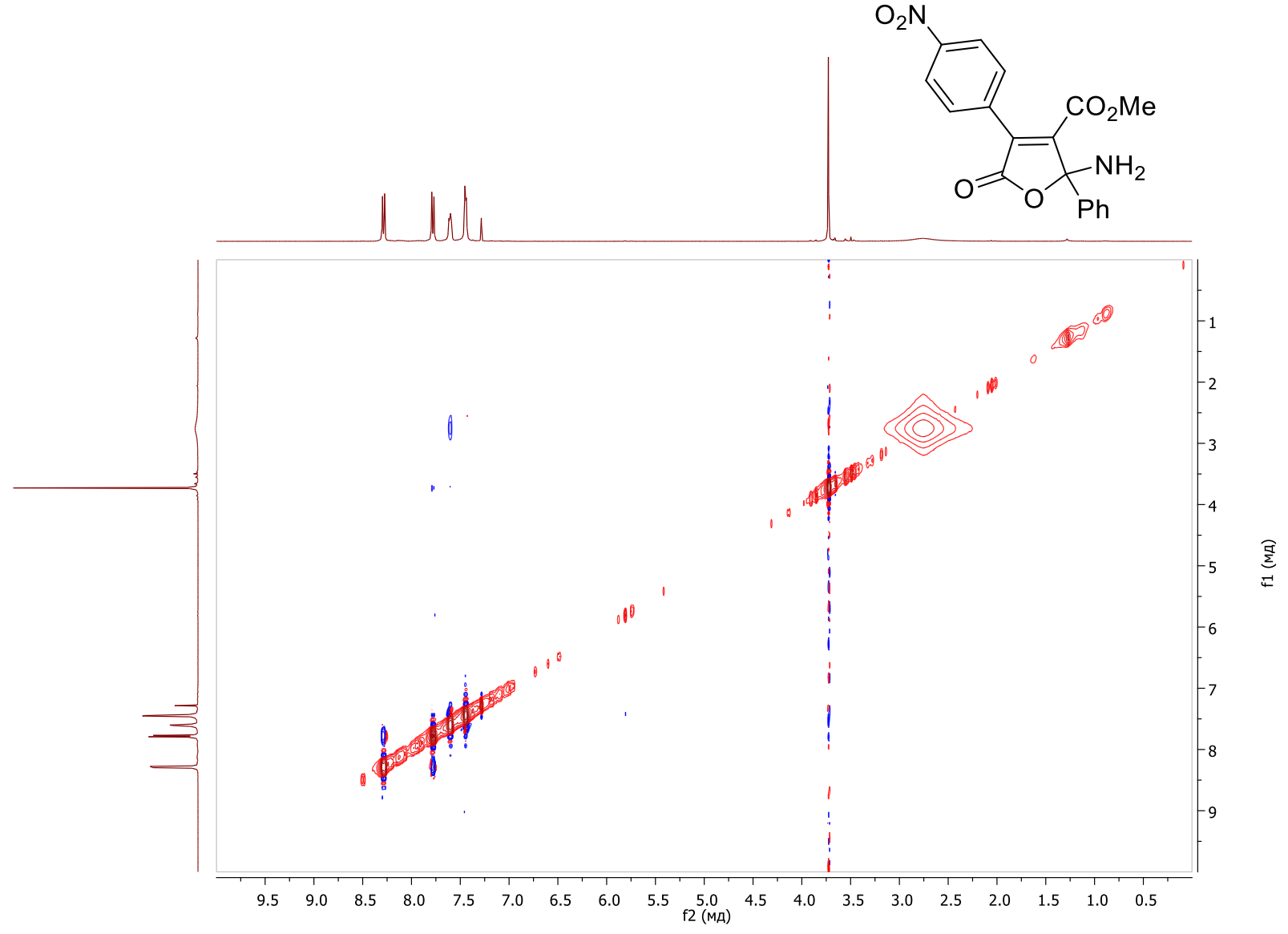


${ }^{1} \mathrm{H}\left(400 \mathrm{MHz}\right.$, DMSO-d 6 ) and ${ }^{13} \mathrm{C}(100 \mathrm{MHz}$, DMSO-d 6 ) NMR spectra of compound $\mathbf{5 b}$
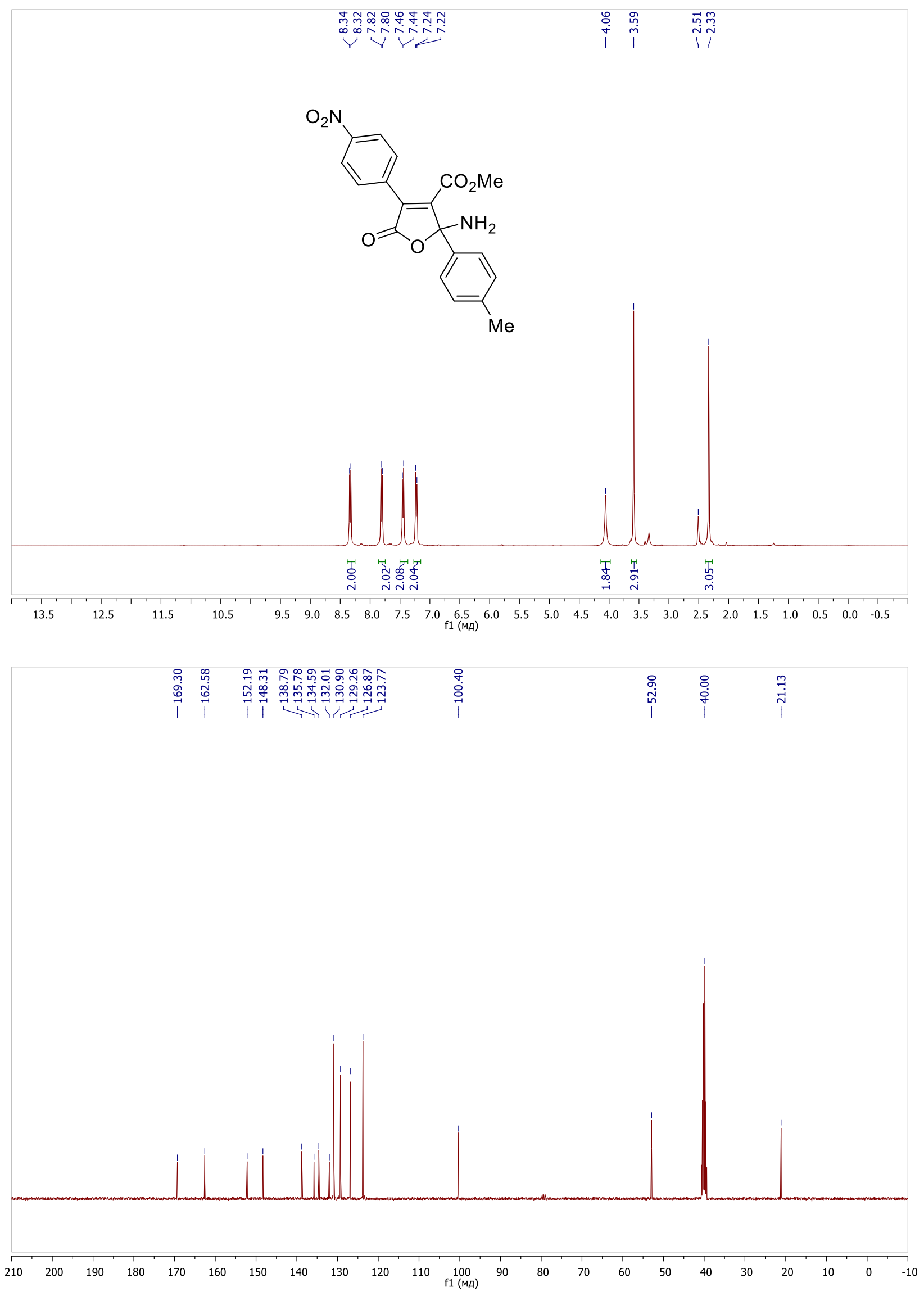

S70 
${ }^{1} \mathrm{H}\left(400 \mathrm{MHz}, \mathrm{CDCl}_{3}\right)$ and ${ }^{13} \mathrm{C}\left(100 \mathrm{MHz}, \mathrm{CDCl}_{3}\right) \mathrm{NMR}$ spectra of compound $\mathbf{5 c}$
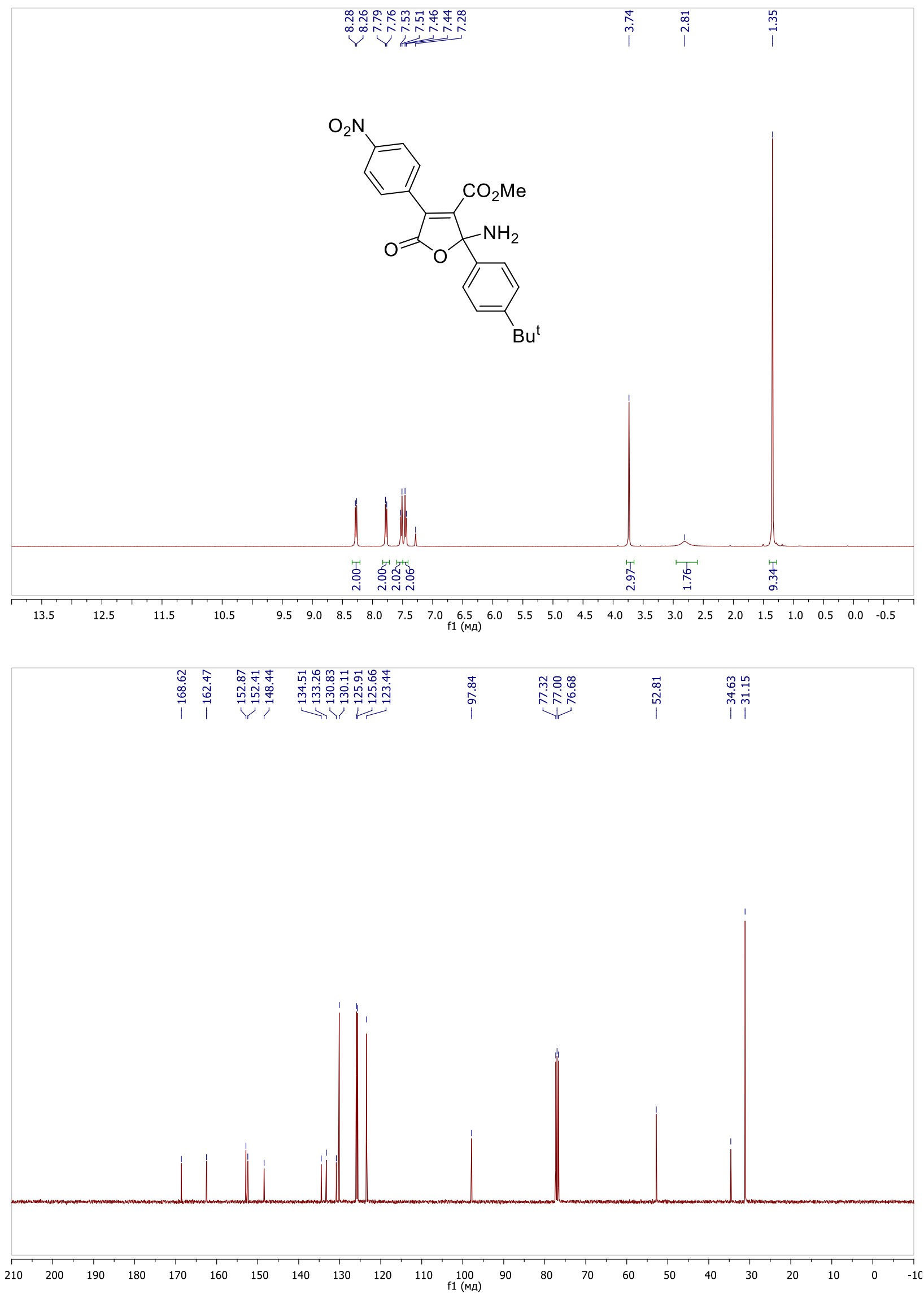
${ }^{1} \mathrm{H}\left(400 \mathrm{MHz}, \mathrm{CDCl}_{3}-\mathrm{DMSO}-\mathrm{d}_{6}\right.$ mixture $)$ and ${ }^{13} \mathrm{C}\left(100 \mathrm{MHz}, \mathrm{CDCl}_{3}-\mathrm{DMSO}_{6} \mathrm{~d}_{6}\right.$ mixture $)$ NMR spectra of compound $\mathbf{5 d}$
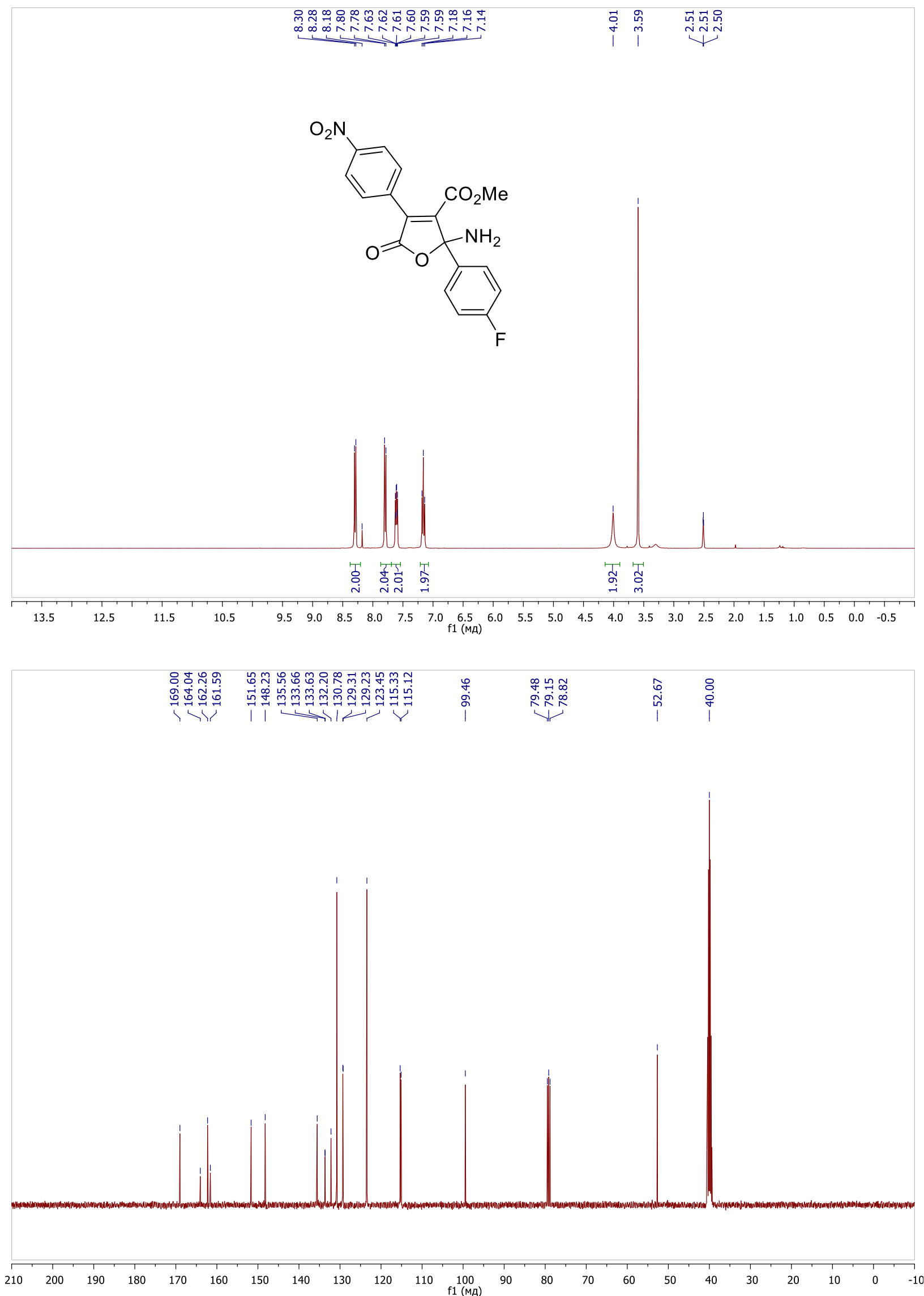
${ }^{1} \mathrm{H}\left(400 \mathrm{MHz}, \mathrm{CDCl}_{3}-\mathrm{DMSO}-\mathrm{d}_{6}\right.$ mixture $)$ and ${ }^{13} \mathrm{C}\left(100 \mathrm{MHz}, \mathrm{CDCl}_{3}-\mathrm{DMSO}^{-\mathrm{d}_{6}}\right.$ mixture $)$ NMR spectra of compound $\mathbf{5 e}$

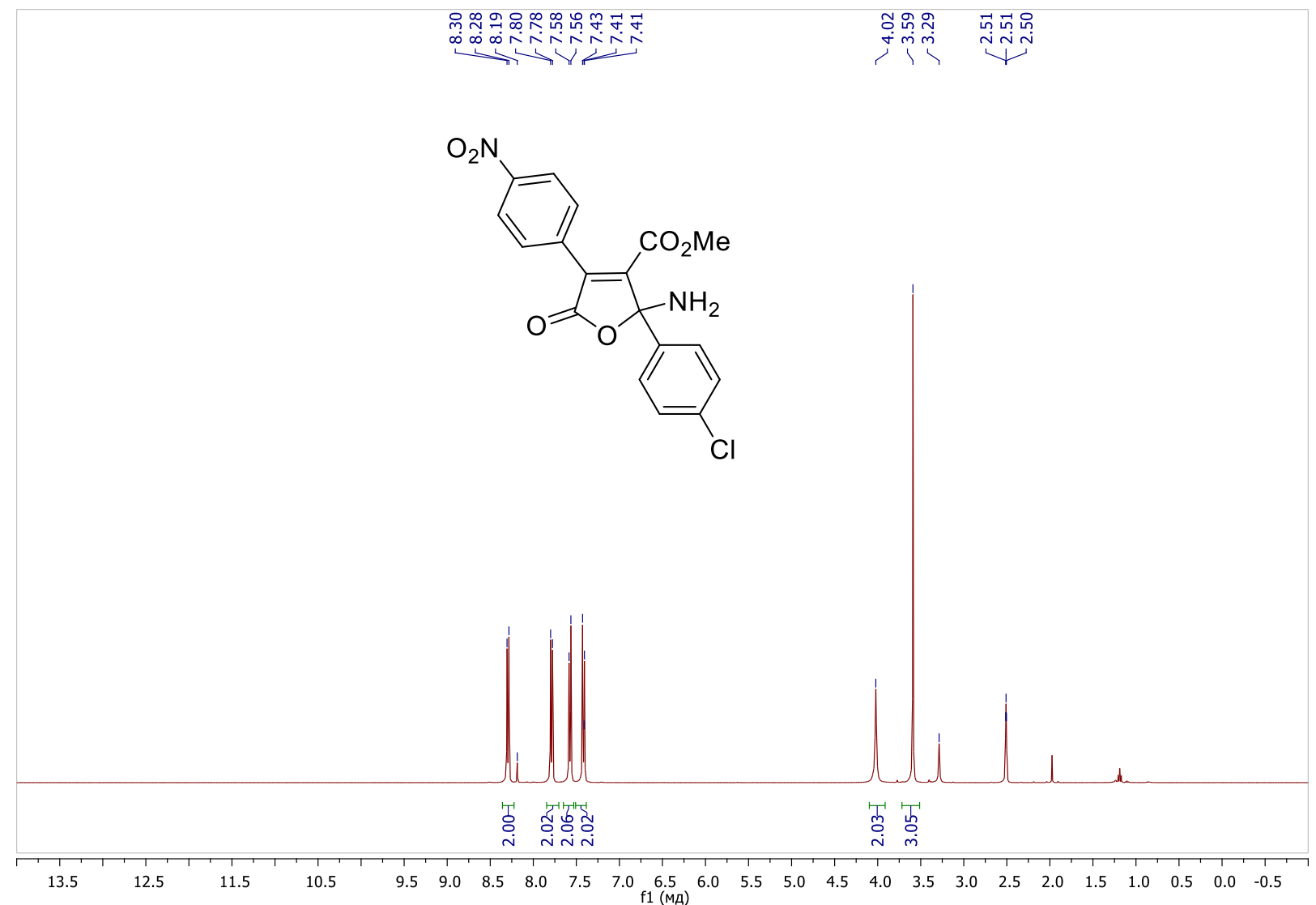

\begin{tabular}{|c|c|c|c|c|c|}
\hline 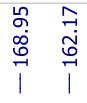 & 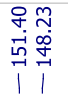 & 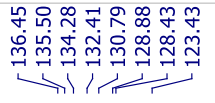 & $\begin{array}{l}\text { mे } \\
\text { gू } \\
1\end{array}$ & 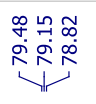 & 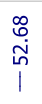 \\
\hline
\end{tabular}

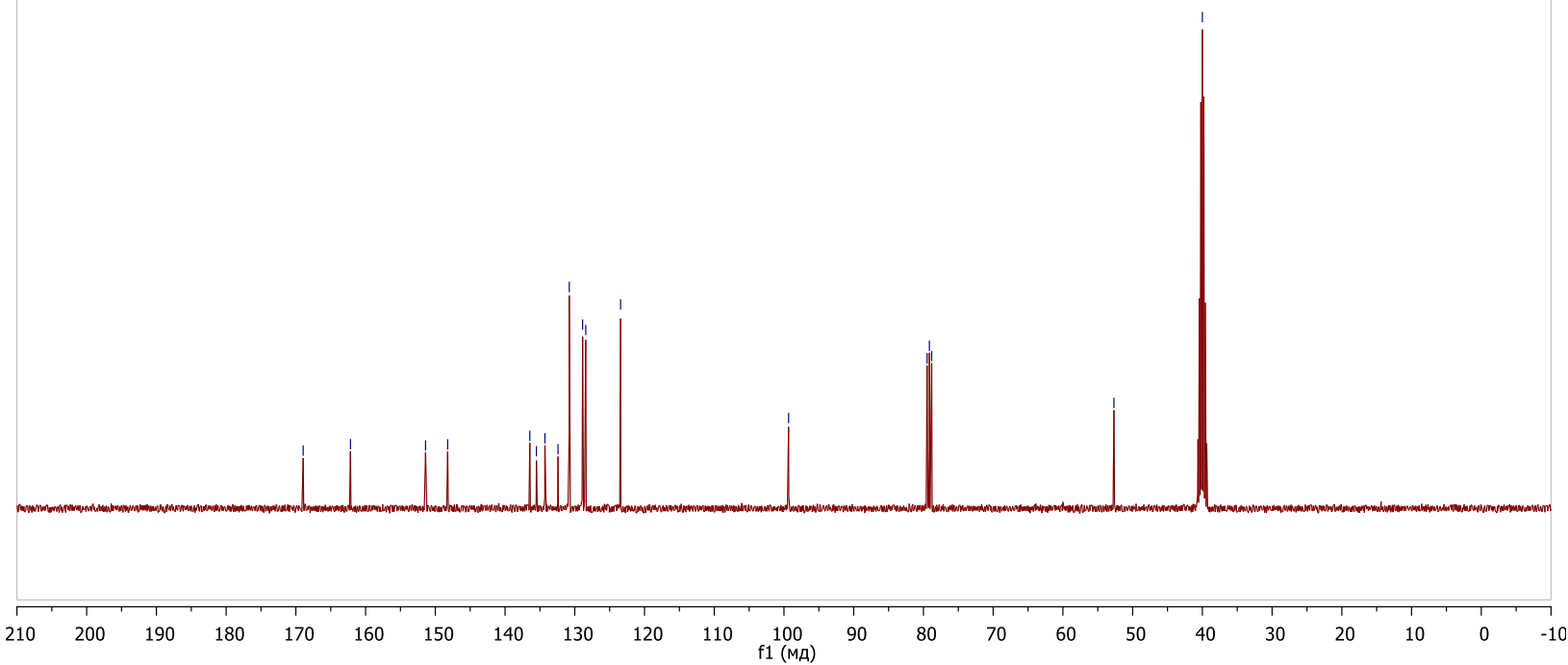


${ }^{1} \mathrm{H}\left(400 \mathrm{MHz}, \mathrm{CDCl}_{3}-\mathrm{DMSO}-\mathrm{d}_{6}\right.$ mixture $)$ and ${ }^{13} \mathrm{C}\left(100 \mathrm{MHz}, \mathrm{CDCl}_{3}-\mathrm{DMSO}^{-\mathrm{d}_{6}}\right.$ mixture $)$ NMR spectra of compound $\mathbf{5 f}$
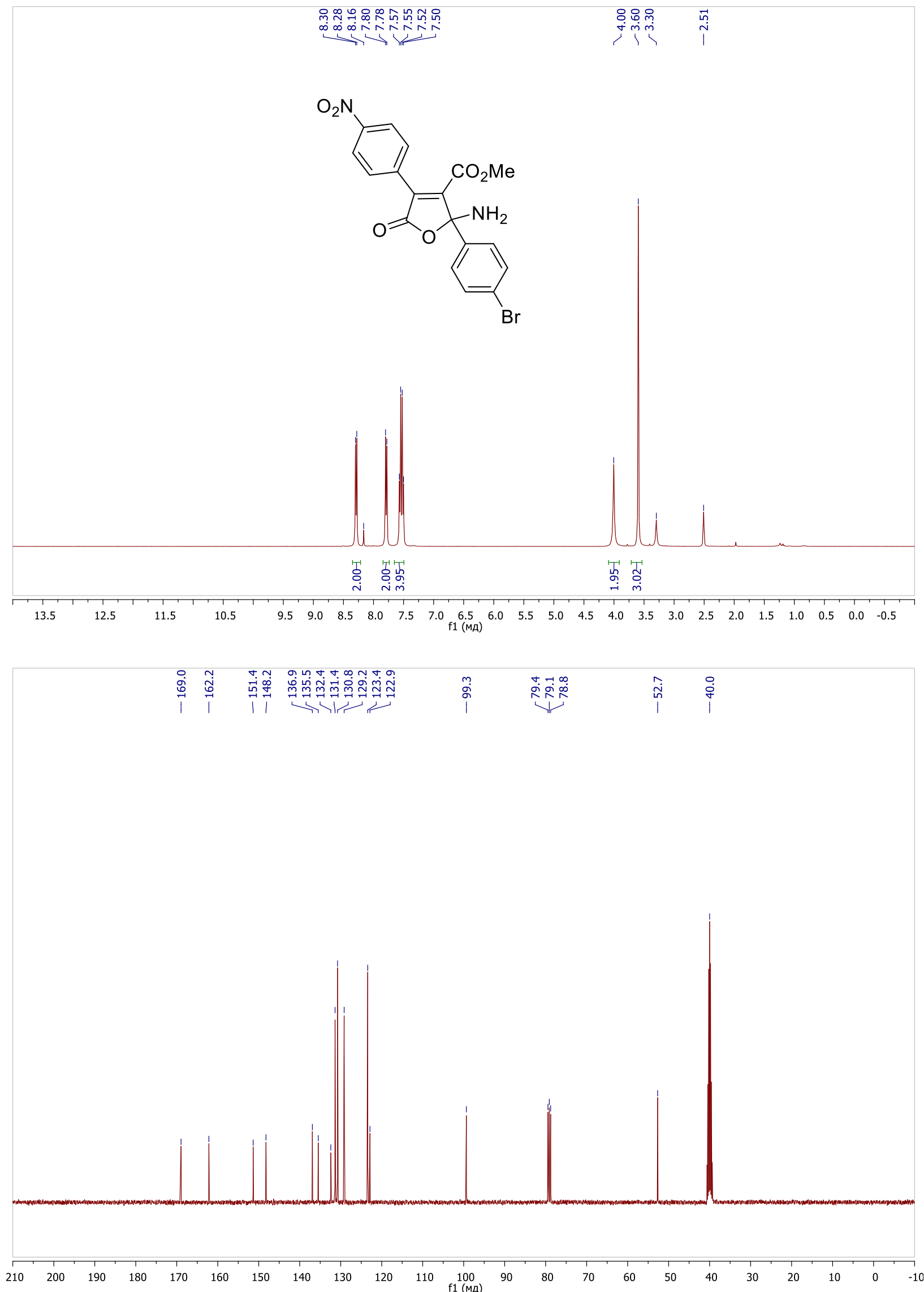
${ }^{1} \mathrm{H}\left(400 \mathrm{MHz}, \mathrm{CDCl}_{3}-\mathrm{DMSO}-\mathrm{d}_{6}\right.$ mixture $)$ and ${ }^{13} \mathrm{C}\left(100 \mathrm{MHz}, \mathrm{CDCl}_{3}-\mathrm{DMSO}_{6} \mathrm{~d}_{6}\right.$ mixture $)$ NMR spectra of compound $\mathbf{5 g}$

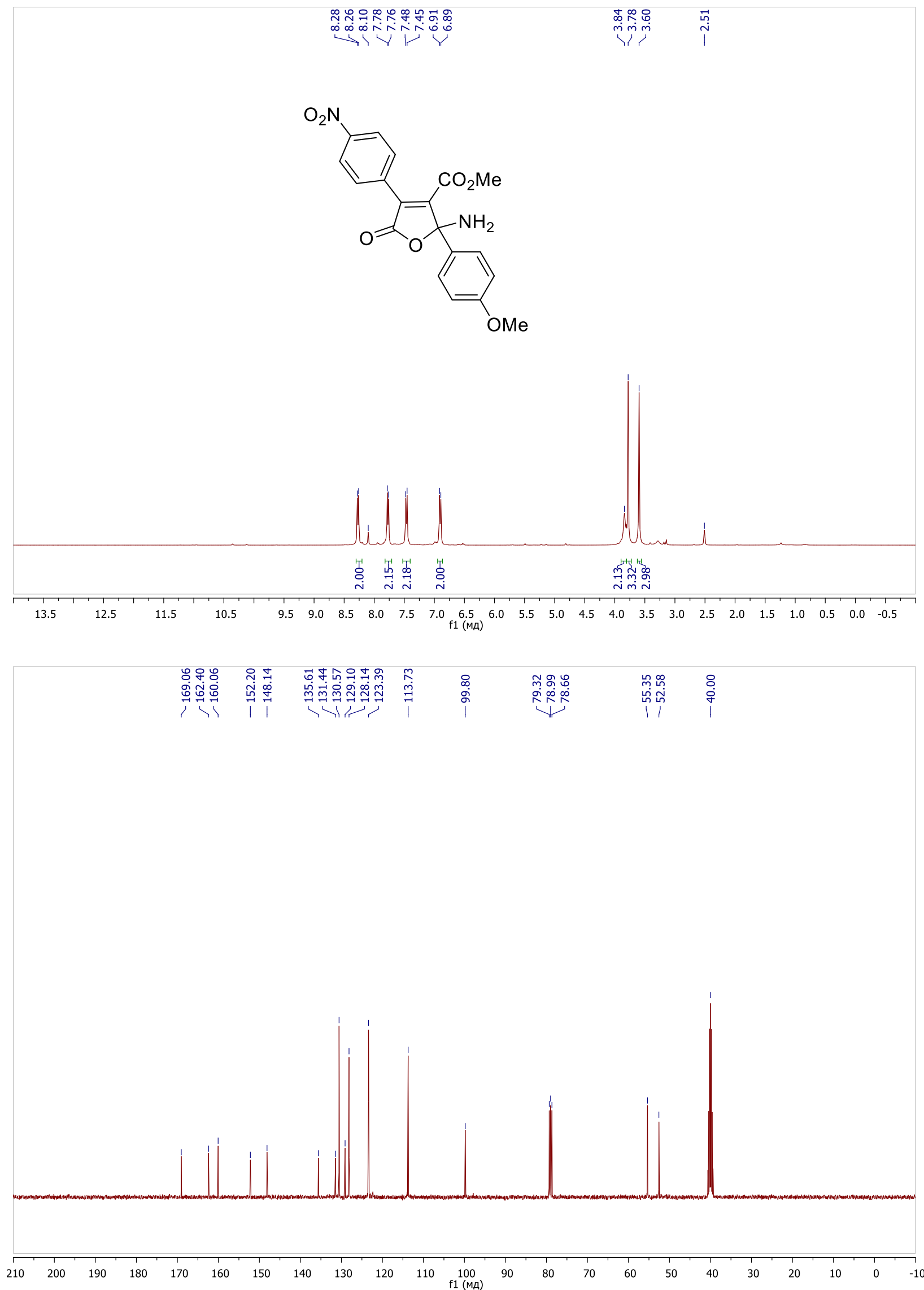


${ }^{1} \mathrm{H}\left(400 \mathrm{MHz}, \mathrm{CDCl}_{3}\right)$ and ${ }^{13} \mathrm{C}\left(100 \mathrm{MHz}, \mathrm{CDCl}_{3}\right)$ NMR spectra of compound $\mathbf{5 h}$
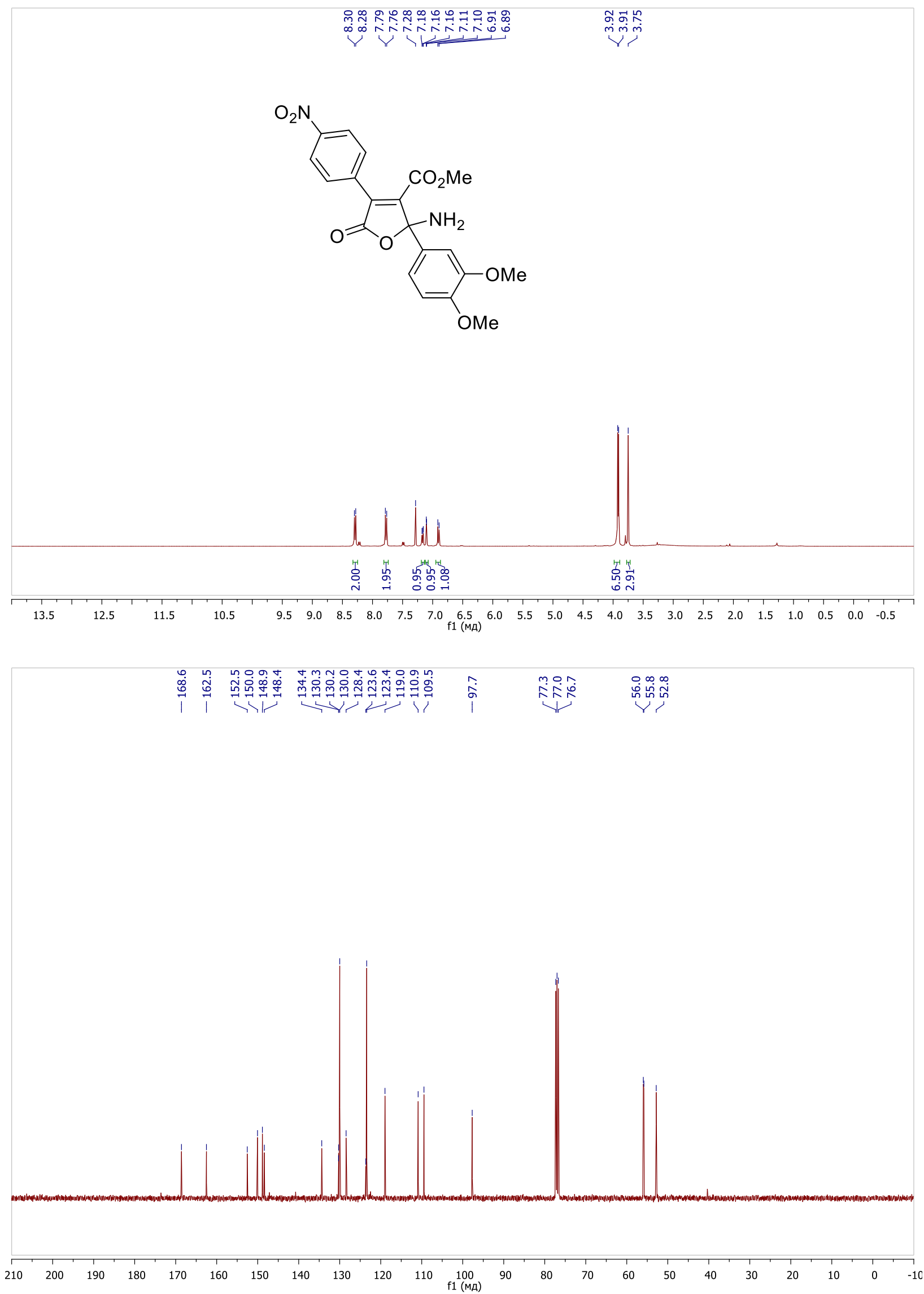
${ }^{1} \mathrm{H}\left(400 \mathrm{MHz}, \mathrm{CDCl}_{3}-\mathrm{DMSO}-\mathrm{d}_{6}\right.$ mixture $)$ and ${ }^{13} \mathrm{C}\left(100 \mathrm{MHz}, \mathrm{CDCl}_{3}-\mathrm{DMSO}^{-\mathrm{d}_{6}}\right.$ mixture $)$ NMR spectra of compound $\mathbf{5 i}$
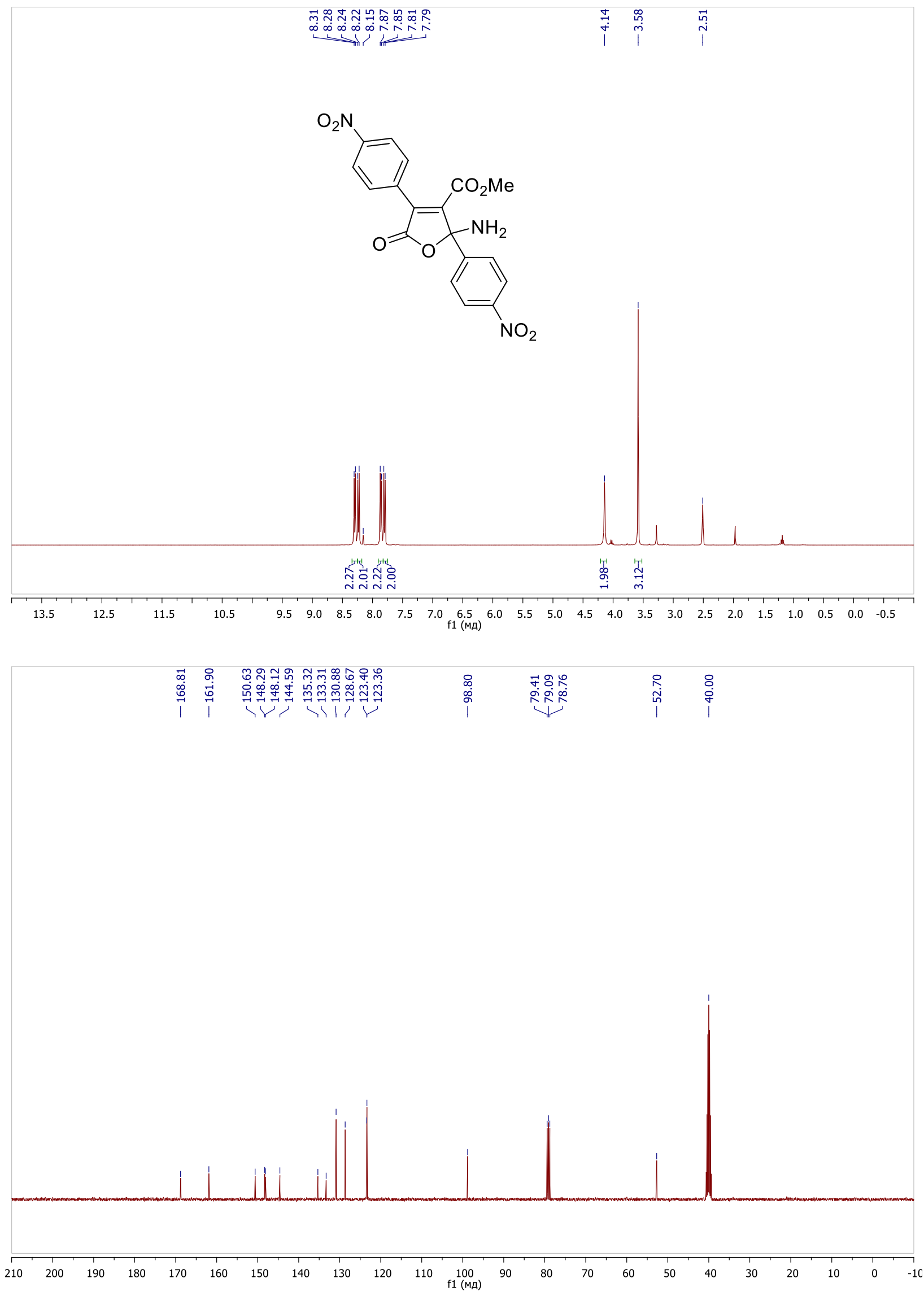
${ }^{1} \mathrm{H}\left(400 \mathrm{MHz}, \mathrm{CDCl}_{3}\right)$ and ${ }^{13} \mathrm{C}\left(100 \mathrm{MHz}, \mathrm{CDCl}_{3}\right)$ NMR spectra of compound $\mathbf{5 j}$
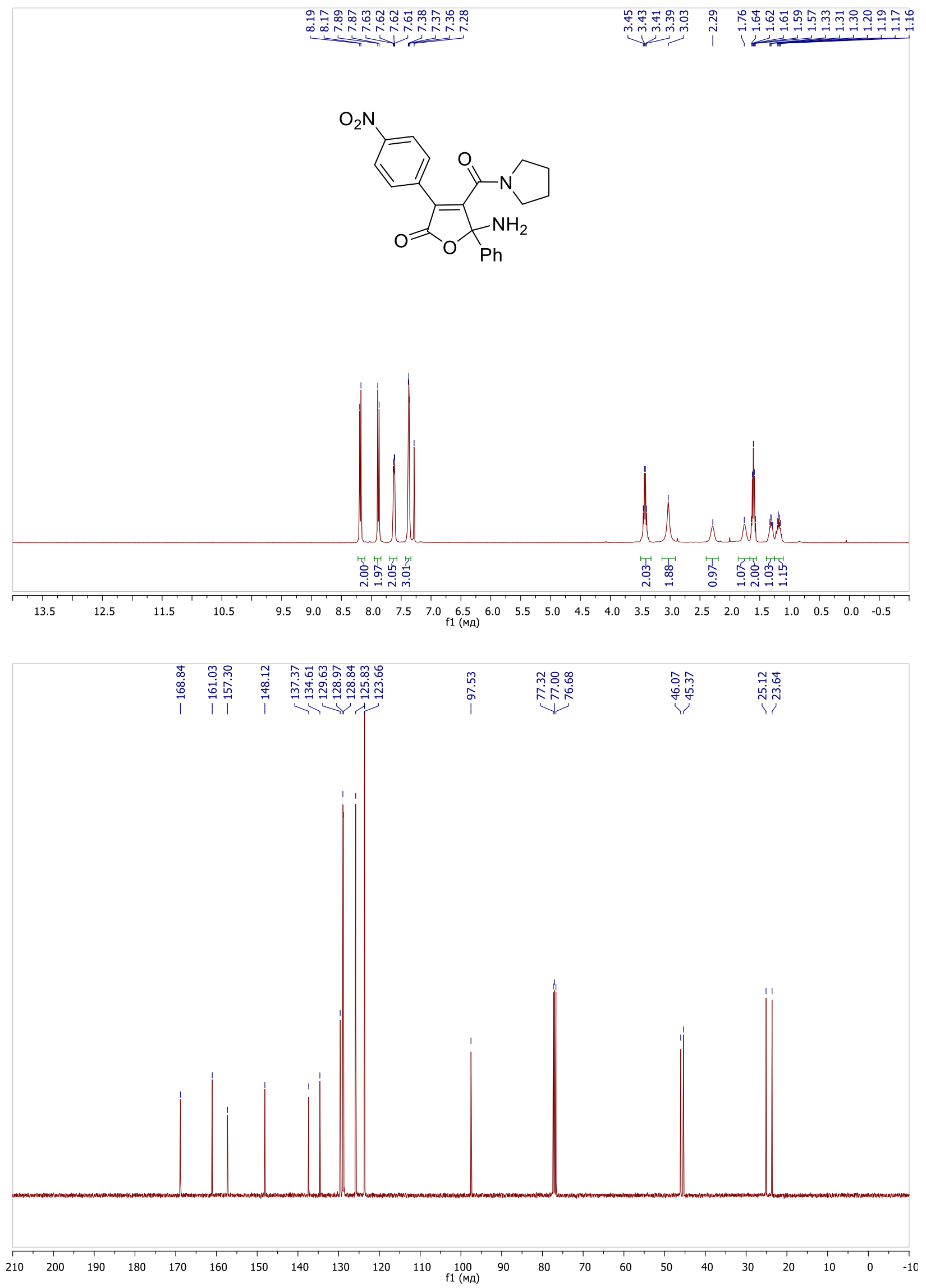
HMBC ${ }^{1} \mathrm{H}^{13}{ }^{13} \mathrm{C}$ NMR spectrum $\left(\mathrm{CDCl}_{3}\right)$ of compound $\mathbf{5} \mathbf{j}$

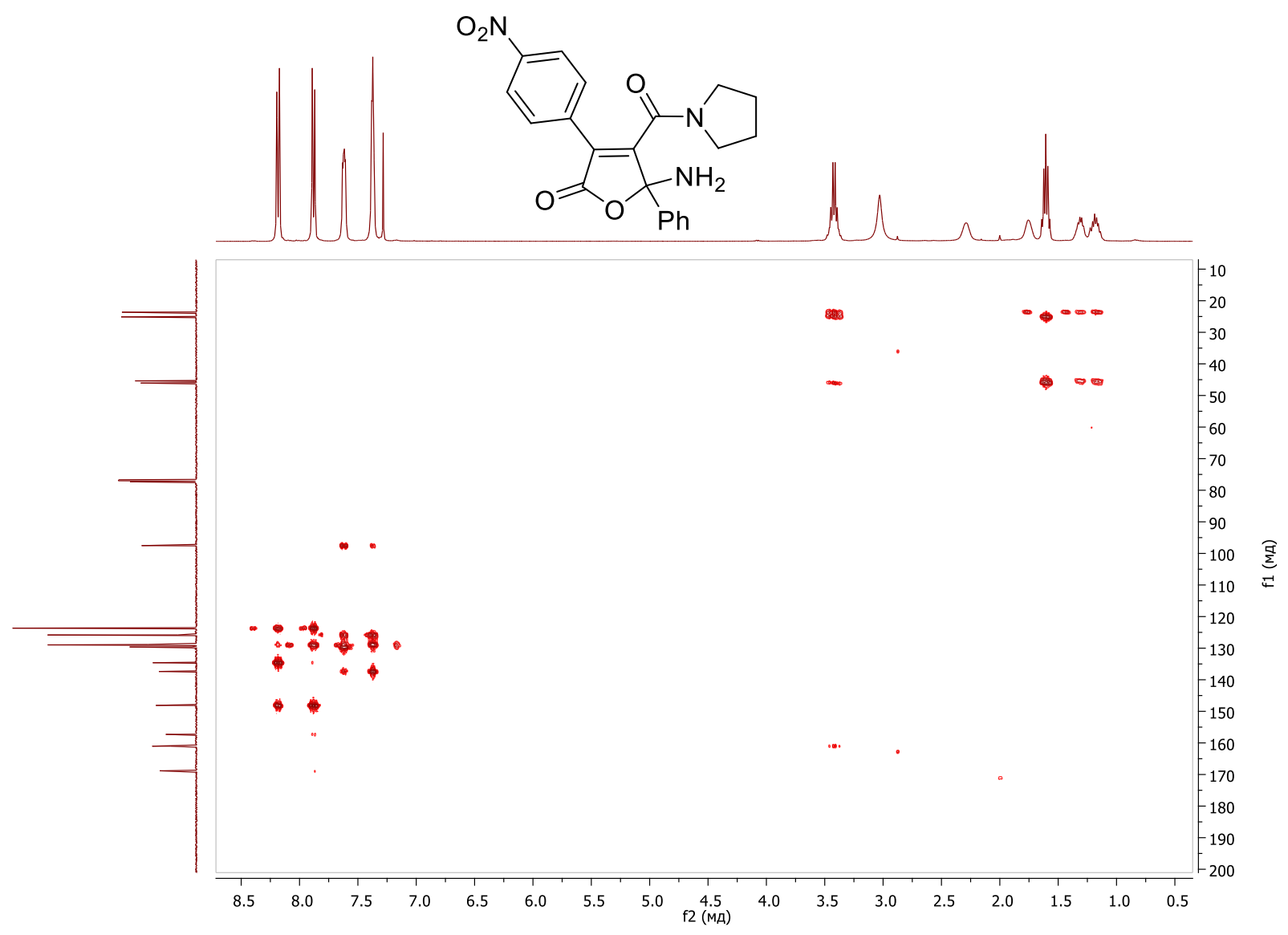


${ }^{1} \mathrm{H}\left(400 \mathrm{MHz}, \mathrm{CDCl}_{3}\right)$ and ${ }^{13} \mathrm{C}\left(100 \mathrm{MHz}, \mathrm{CDCl}_{3}\right) \mathrm{NMR}$ spectra of compound $\mathbf{5 k}$
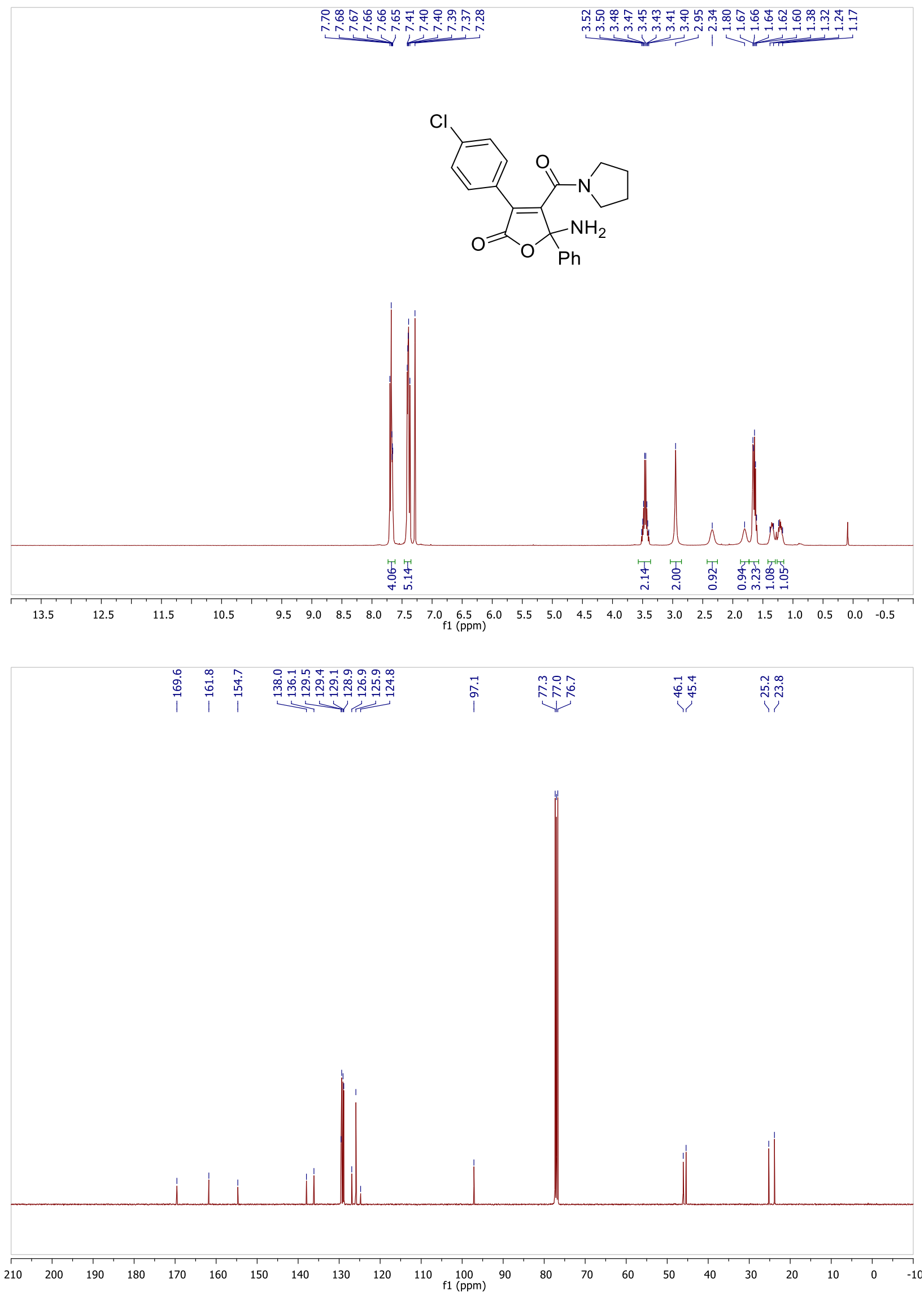
HMBC ${ }^{1} \mathrm{H}^{-13} \mathrm{C}\left(400 \mathrm{MHz}, \mathrm{CDCl}_{3}\right)$ NMR spectrum of compound $\mathbf{5 k}$

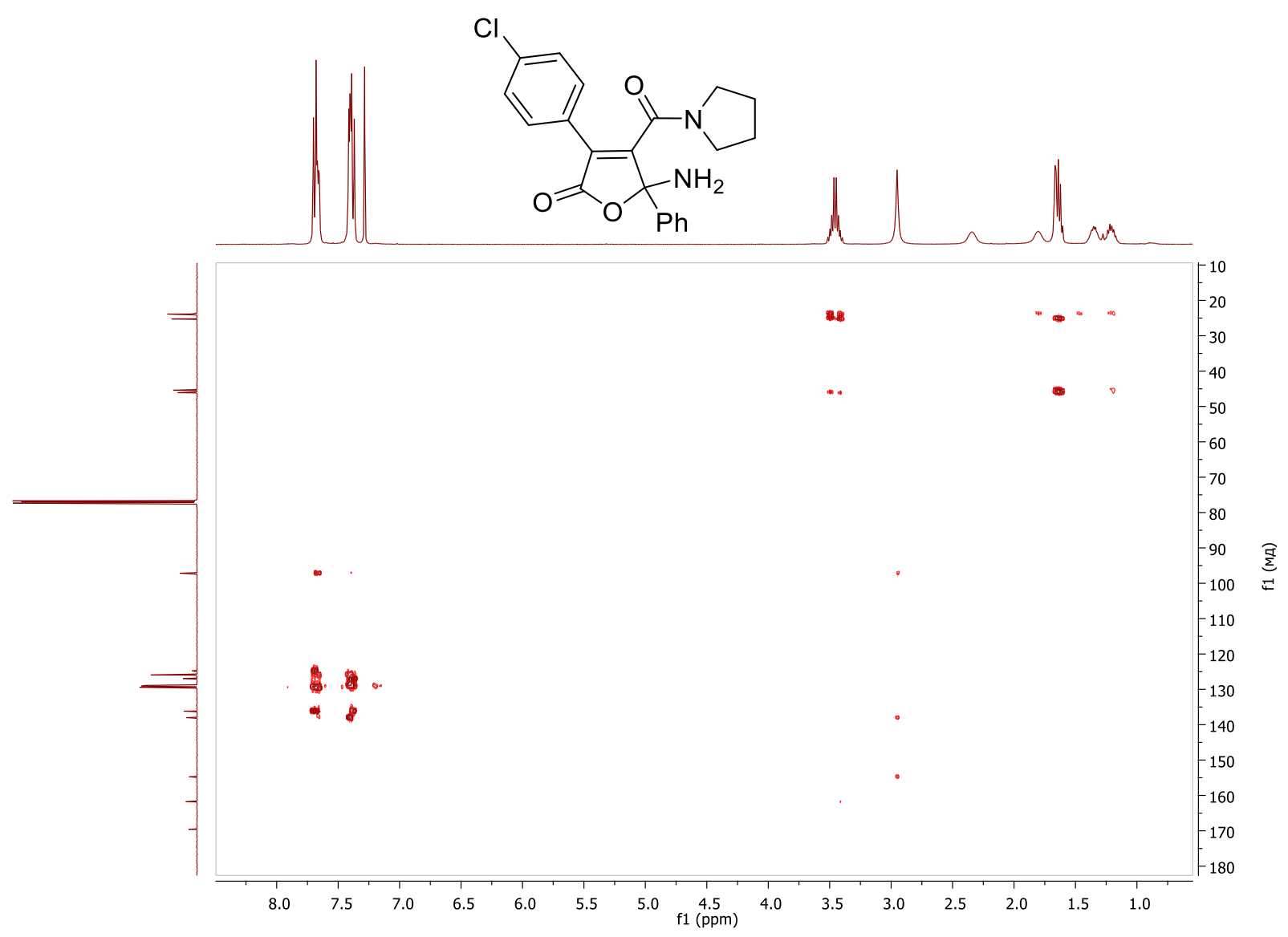


${ }^{1} \mathrm{H}\left(400 \mathrm{MHz}, \mathrm{CDCl}_{3}\right)$ and ${ }^{13} \mathrm{C}\left(100 \mathrm{MHz}, \mathrm{CDCl}_{3}\right)$ NMR spectra of compound $\mathbf{5 l}$
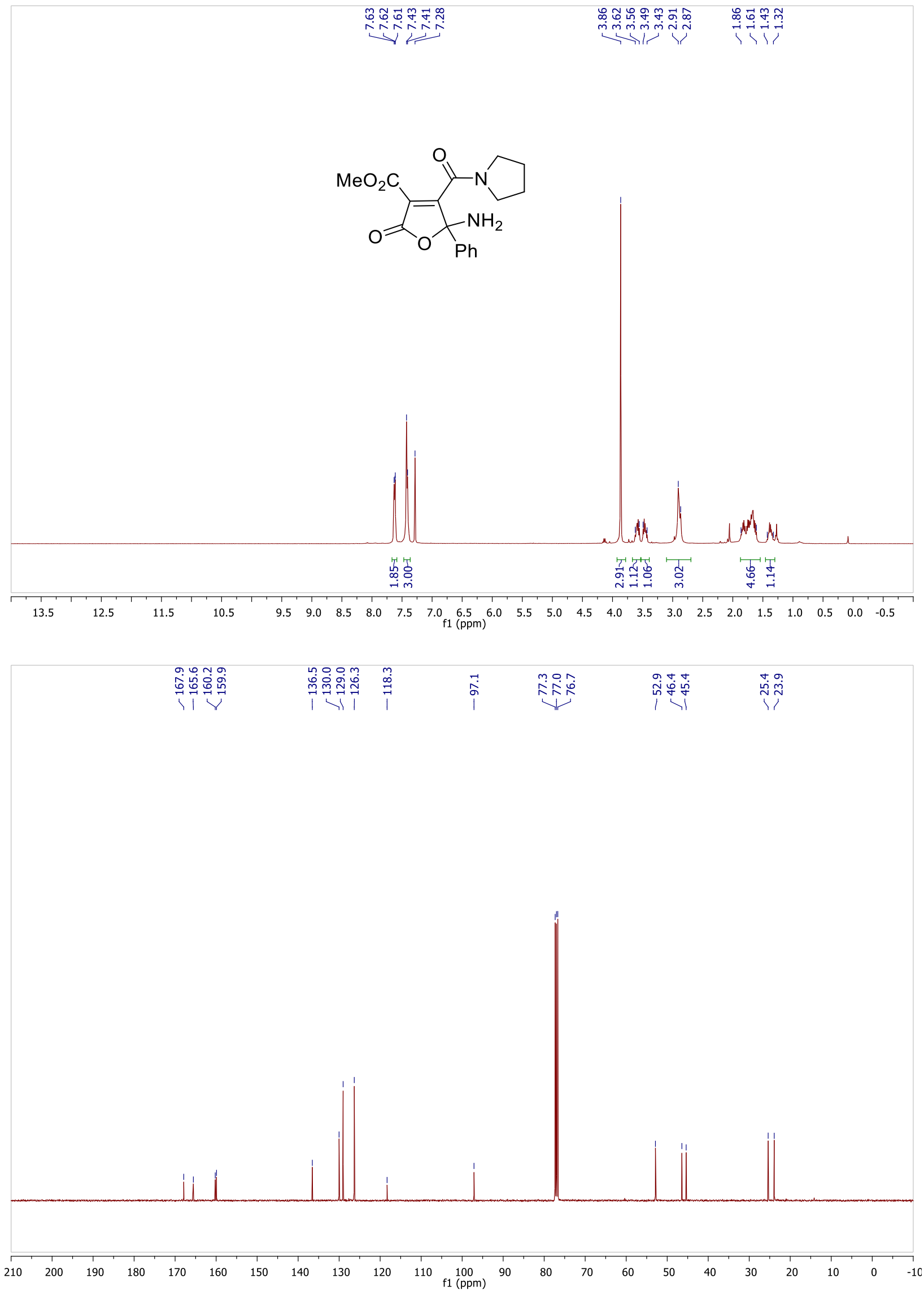
HMBC ${ }^{1} \mathrm{H}^{-13} \mathrm{C}\left(400 \mathrm{MHz}, \mathrm{CDCl}_{3}\right)$ NMR spectrum of compound $\mathbf{5 l}$

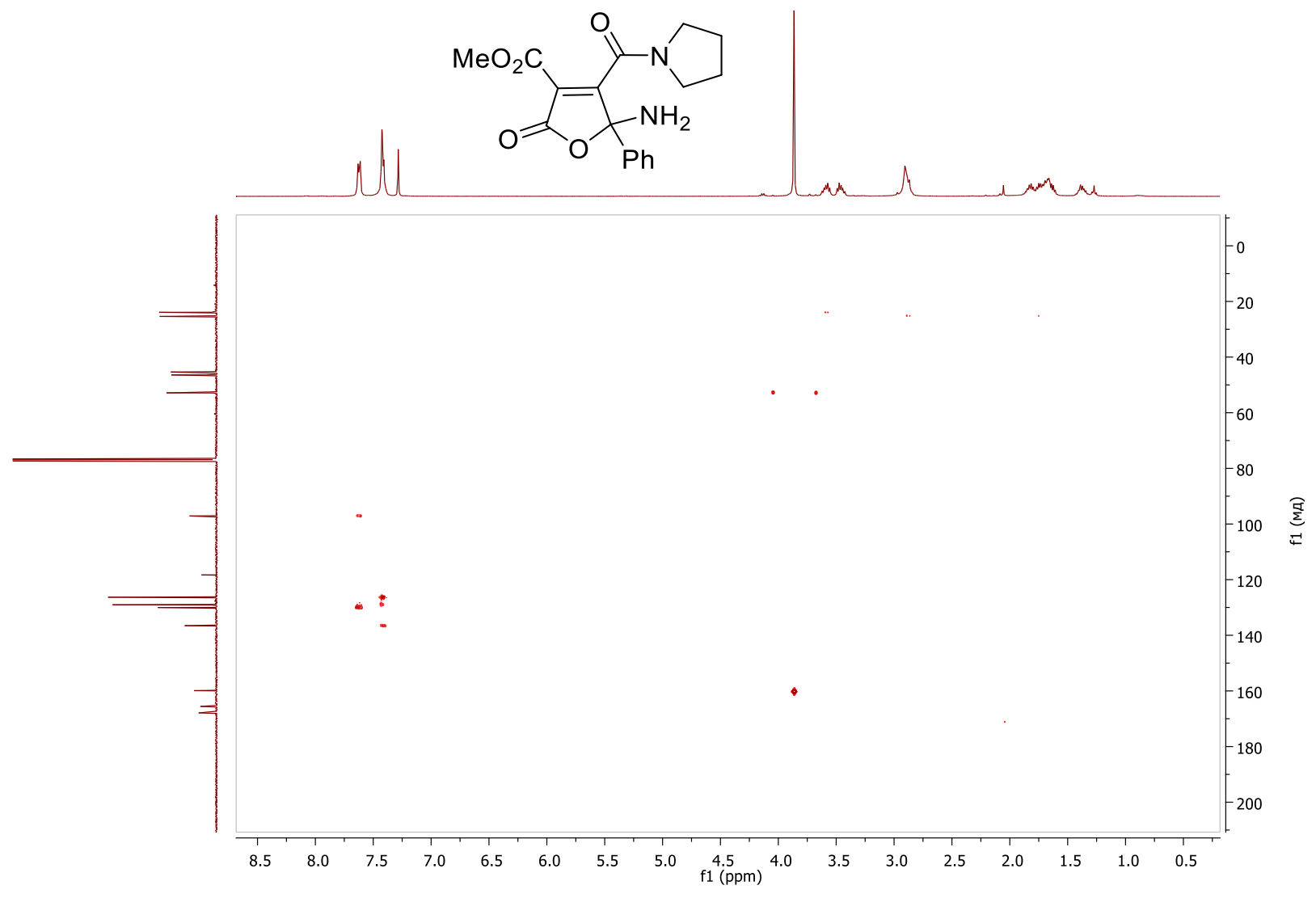


${ }^{1} \mathrm{H}\left(400 \mathrm{MHz}, \mathrm{CDCl}_{3}\right)$ and ${ }^{13} \mathrm{C}\left(100 \mathrm{MHz}, \mathrm{CDCl}_{3}\right)$ NMR spectra of compound $\mathbf{5 m}$
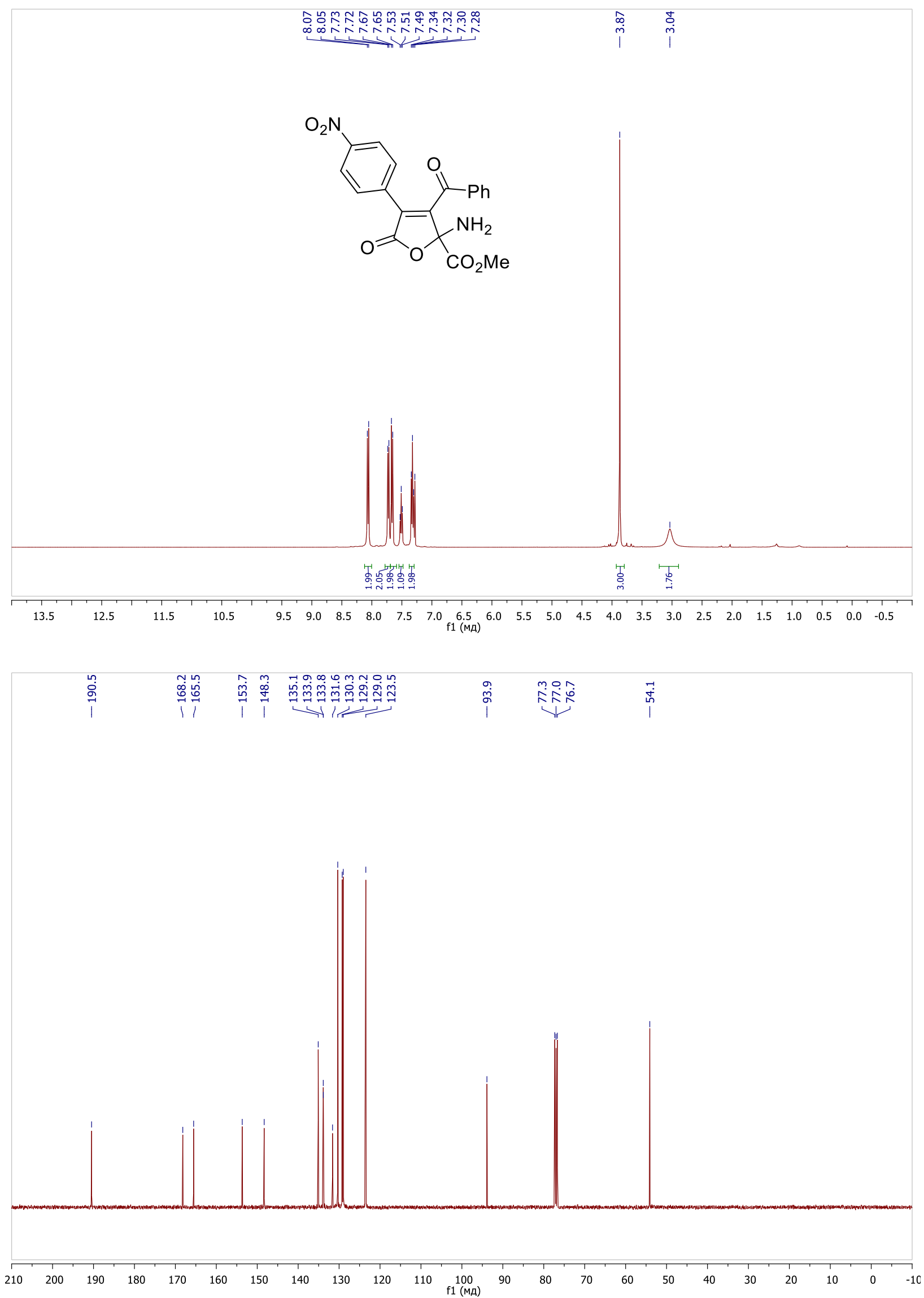
HMBC ${ }^{1} \mathrm{H}^{13} \mathrm{C}^{13} \mathrm{NR}$ spectrum $\left(\mathrm{CDCl}_{3}\right)$ of compound $\mathbf{5 m}$

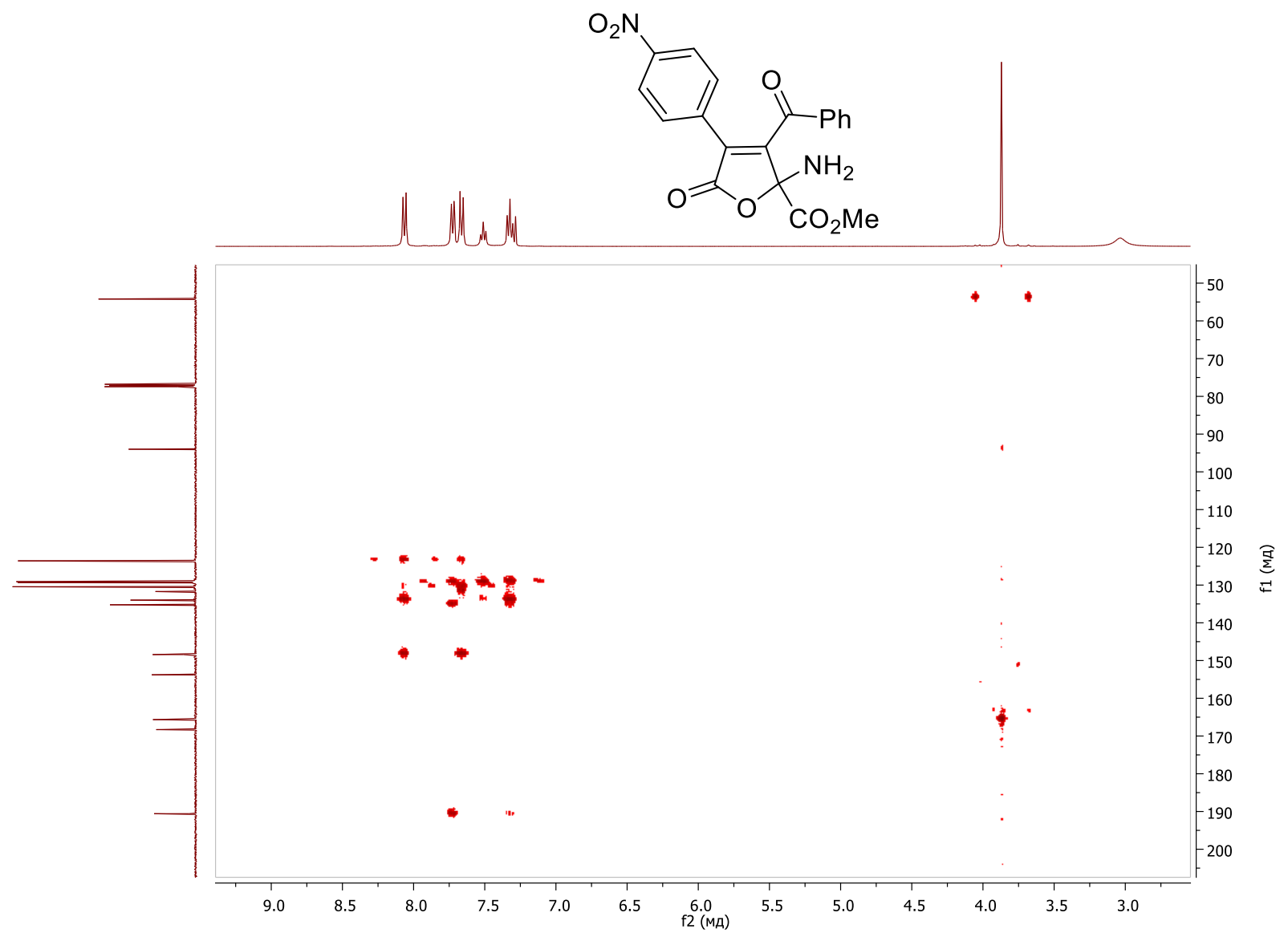


${ }^{1} \mathrm{H}\left(400 \mathrm{MHz}, \mathrm{CDCl}_{3}\right)$ and ${ }^{13} \mathrm{C}\left(100 \mathrm{MHz}, \mathrm{CDCl}_{3}\right)$ NMR spectra of compound $\mathbf{1 2 d}$
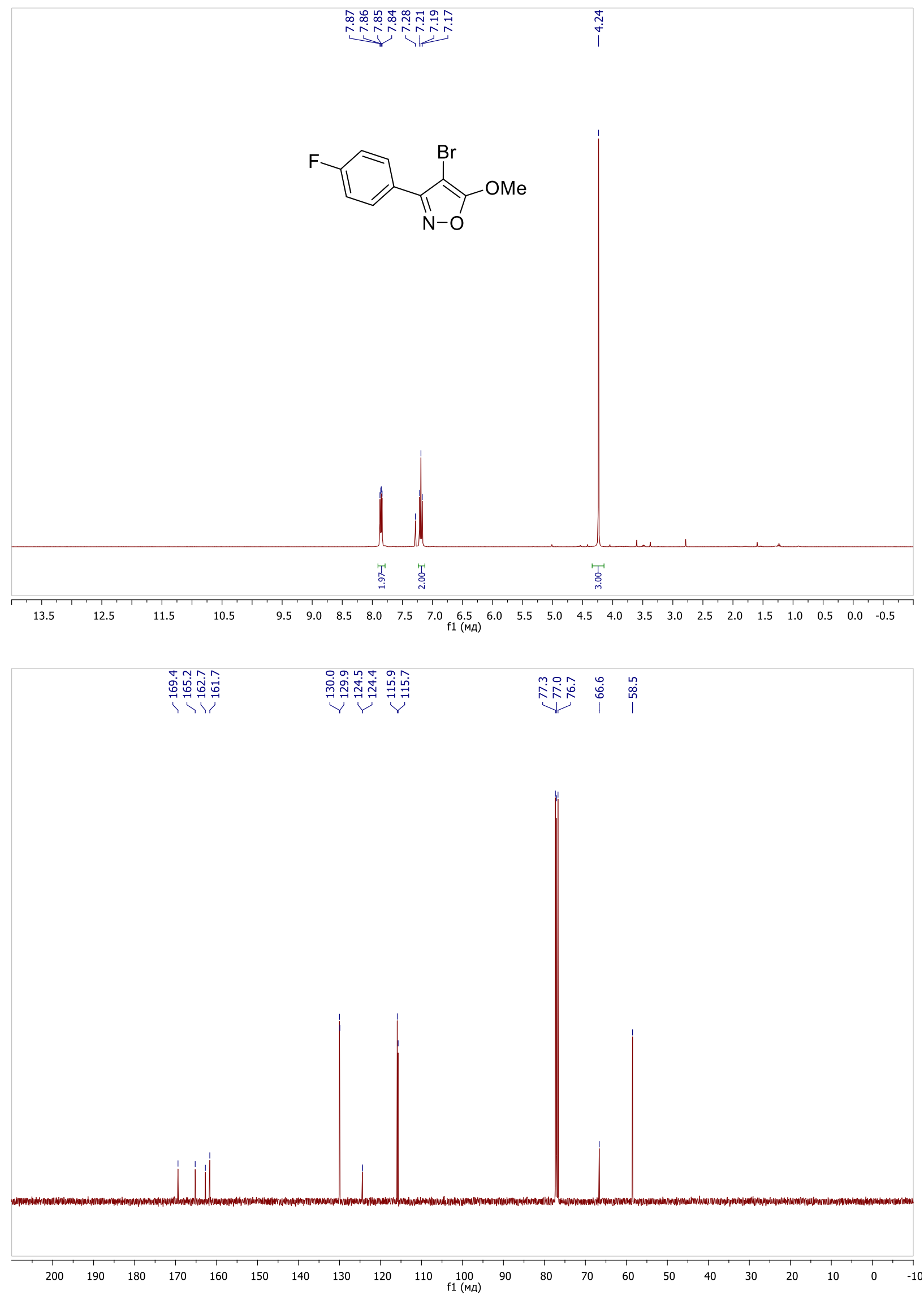
${ }^{1} \mathrm{H}\left(400 \mathrm{MHz}, \mathrm{CDCl}_{3}\right)$ and ${ }^{13} \mathrm{C}\left(100 \mathrm{MHz}, \mathrm{CDCl}_{3}\right)$ NMR spectra of compound $\mathbf{1 2 h}$

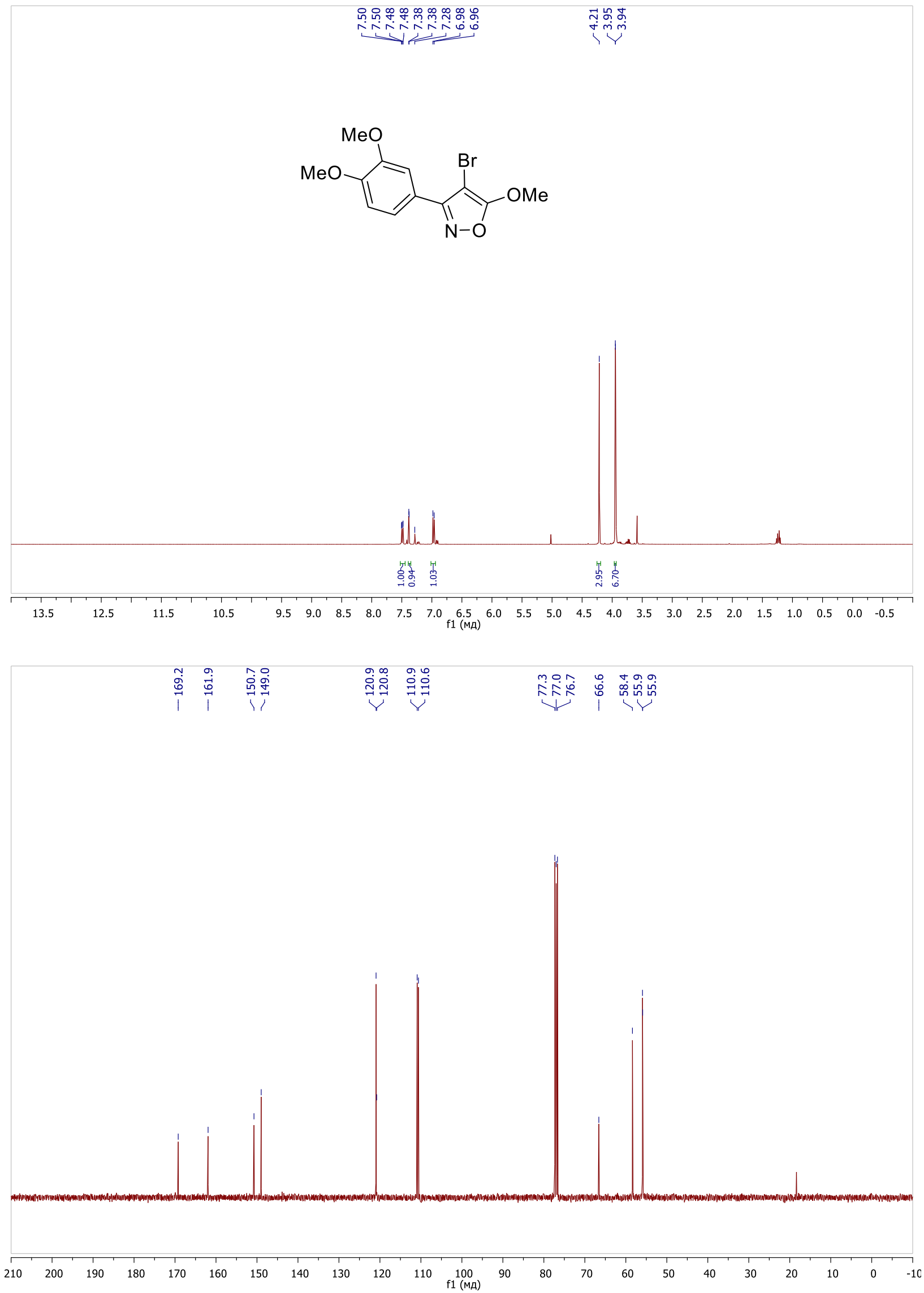


${ }^{1} \mathrm{H}\left(400 \mathrm{MHz}, \mathrm{CDCl}_{3}\right)$ and ${ }^{13} \mathrm{C}\left(100 \mathrm{MHz}, \mathrm{CDCl}_{3}\right)$ NMR spectra of compound $\mathbf{1 2 n}$

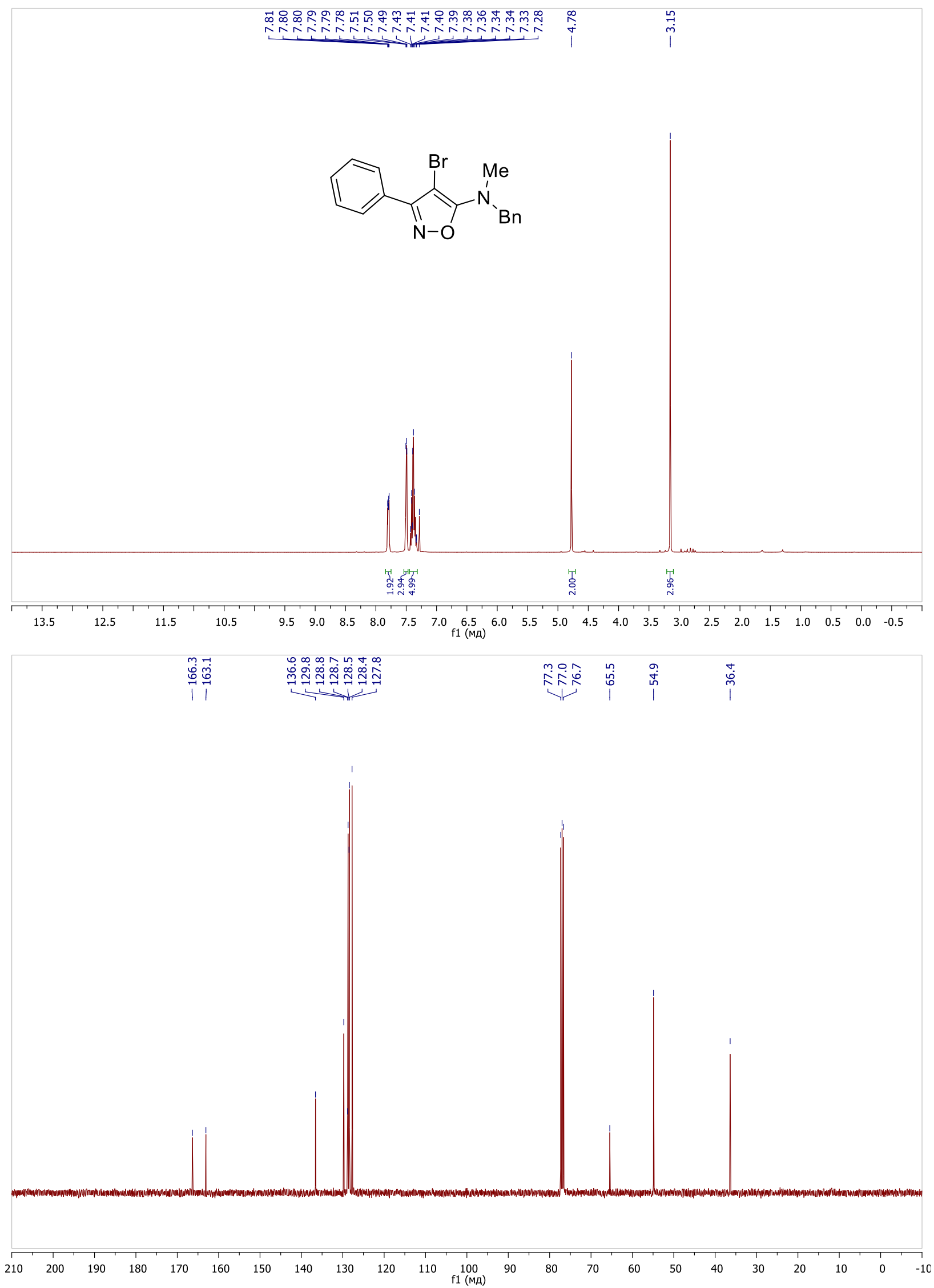


${ }^{1} \mathrm{H}\left(400 \mathrm{MHz}, \mathrm{CDCl}_{3}\right)$ and ${ }^{13} \mathrm{C}\left(100 \mathrm{MHz}, \mathrm{CDCl}_{3}\right)$ NMR spectra of compound 12p

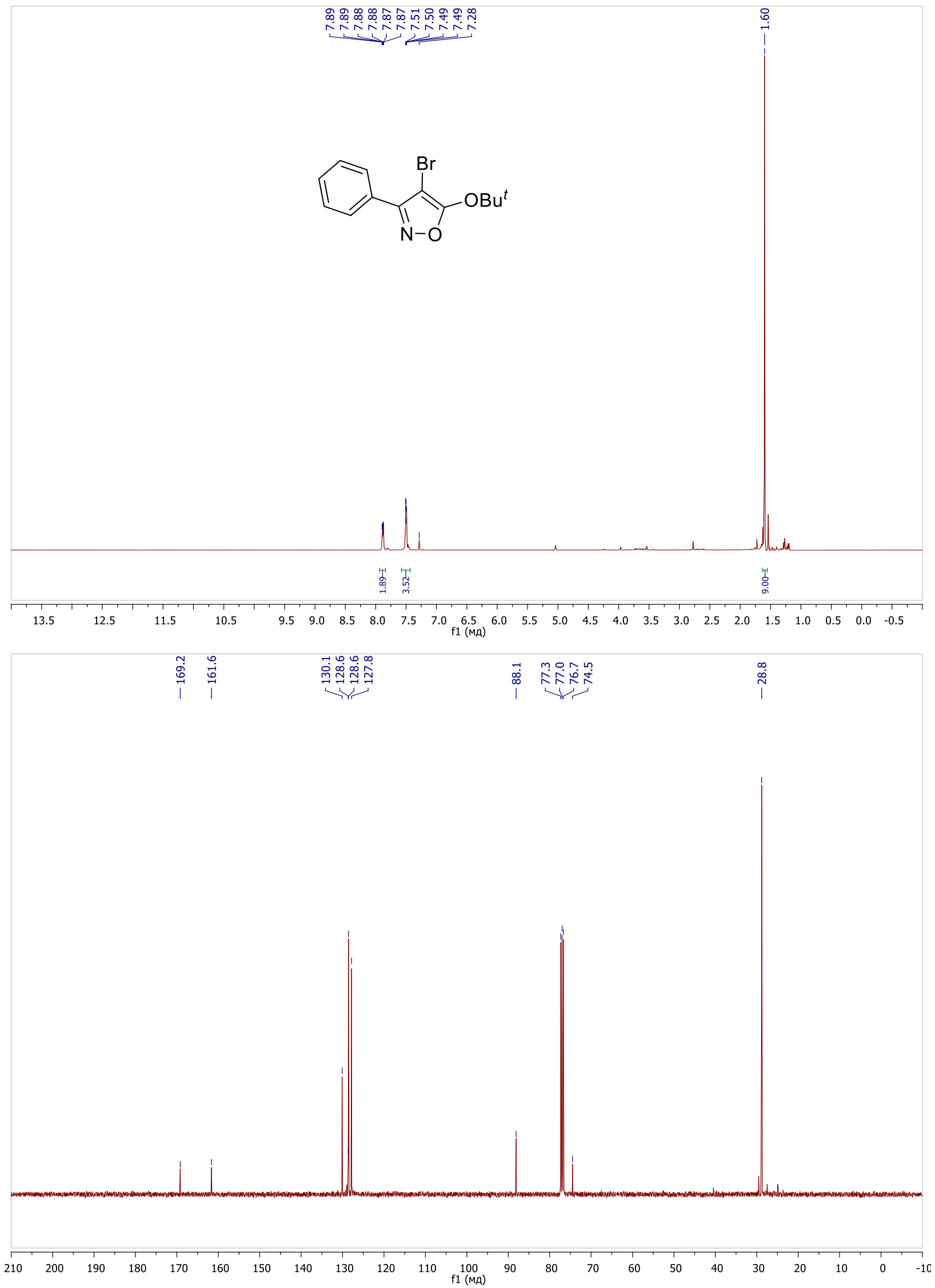




\section{X-ray data}

Compound 4o. Single crystals of 40 were grown by slow evaporation of its solution in $\mathrm{CHCl}_{3}$ hexane mixture. A suitable crystal was selected and intensity data were collected on a SuperNova (Dual,Cu. at zero, Atlas $(\mathrm{MoK} \alpha)$ ) diffractometer. The crystal was kept at 100(2) K during data collection. Using Olex $2{ }^{8}$ the structure was solved with the Superflip ${ }^{9}$ structure solution program using Charge Flipping and refined with the ShelXL ${ }^{10}$ refinement package using Least Squares minimisation.

Figure S2. X-Ray crystal structure of methyl 2-amino-4-(4-chlorophenyl)-5-oxo-3-phenyl-2,5dihydrofuran-2-carboxylate 40 with 50\% ellipsoid probability (CCDC 1902292)

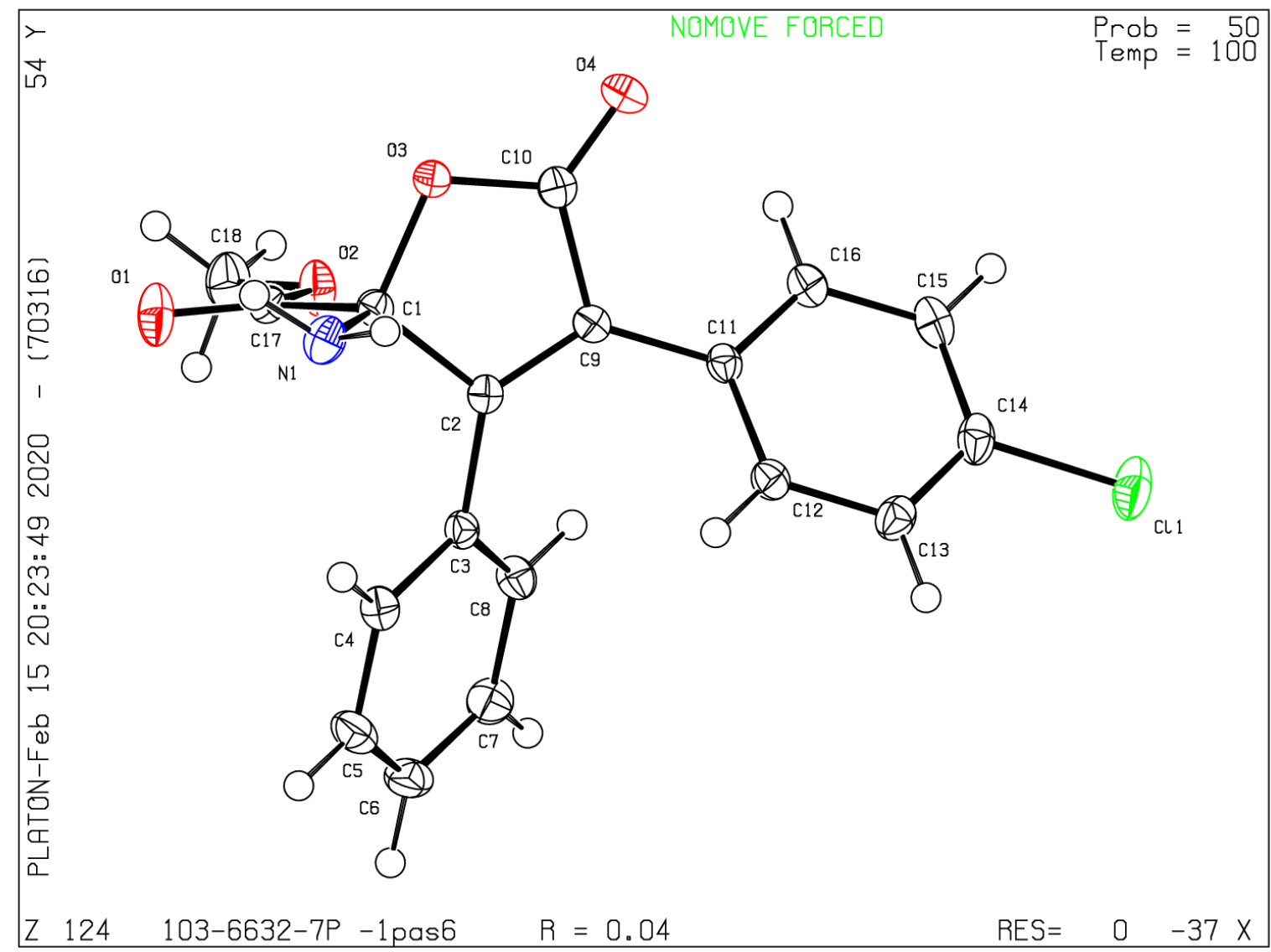


Table S2. Crystal data and structure refinement for 40

Empirical formula

Formula weight

Temperature/K

Crystal system

Space group

$\mathrm{a} / \AA$

$\mathrm{b} / \AA$

$\mathrm{c} / \AA$

$\alpha /{ }^{\circ}$

$\beta /{ }^{\circ}$

$\gamma /{ }^{\circ}$

Volume $/ \AA^{3}$

$\mathrm{Z}$

$\rho_{\text {calc }} \mathrm{g} / \mathrm{cm}^{3}$

$\mu / \mathrm{mm}^{-1}$

$\mathrm{F}(000)$

Crystal size $/ \mathrm{mm}^{3}$

Radiation

$2 \Theta$ range for data collection/ ${ }^{\circ}$

Index ranges

Reflections collected

Independent reflections

Data/restraints/parameters

Goodness-of-fit on $\mathrm{F}^{2}$

Final $\mathrm{R}$ indexes $[\mathrm{I}>=2 \sigma(\mathrm{I})]$

Final R indexes [all data]

Largest diff. peak/hole / e $\AA^{-3}$
$\mathrm{C}_{18} \mathrm{H}_{14} \mathrm{ClNO}_{4}$

343.75

100.01(10)

triclinic

P-1

$5.9960(5)$

$10.5716(6)$

$13.1158(8)$

79.127(5)

$81.979(6)$

$78.549(6)$

795.69(9)

2

1.435

0.262

356.0

$0.52 \times 0.49 \times 0.37$

$\operatorname{MoK} \alpha(\lambda=0.71073)$

6.362 to 54.998

$-7 \leq \mathrm{h} \leq 7,-13 \leq \mathrm{k} \leq 12,-17 \leq 1 \leq 15$

6597

$3654\left[\mathrm{R}_{\mathrm{int}}=0.0337, \mathrm{R}_{\text {sigma }}=0.0528\right]$

$3654 / 0 / 223$

1.036

$\mathrm{R}_{1}=0.0428, \mathrm{wR}_{2}=0.1036$

$\mathrm{R}_{1}=0.0548, \mathrm{wR}_{2}=0.1153$

$0.37 /-0.35$ 
Compound 5a. Single crystals of $\mathbf{5 a}$ were grown by slow evaporation of its solution in $\mathrm{Et}_{2} \mathrm{O}$ hexane-EtOAc mixture. A suitable crystal was selected and intensity data were collected on a SuperNova (Dual,Cu. at zero, Atlas $(\mathrm{CuK \alpha})$ ) diffractometer. The crystal was kept at 100.01(10) $\mathrm{K}$ during data collection. Using Olex $2{ }^{8}$ the structure was solved with the ShelXT ${ }^{11}$ structure solution program using Intrinsic Phasing and refined with the ShelXL ${ }^{10}$ refinement package using Least Squares minimisation.

Figure S3. X-Ray crystal structure of methyl 2-amino-4-(4-nitrophenyl)-5-oxo-2-phenyl-2,5dihydrofuran-3-carboxylate 5a with 50\% ellipsoid probability (CCDC 1986590)

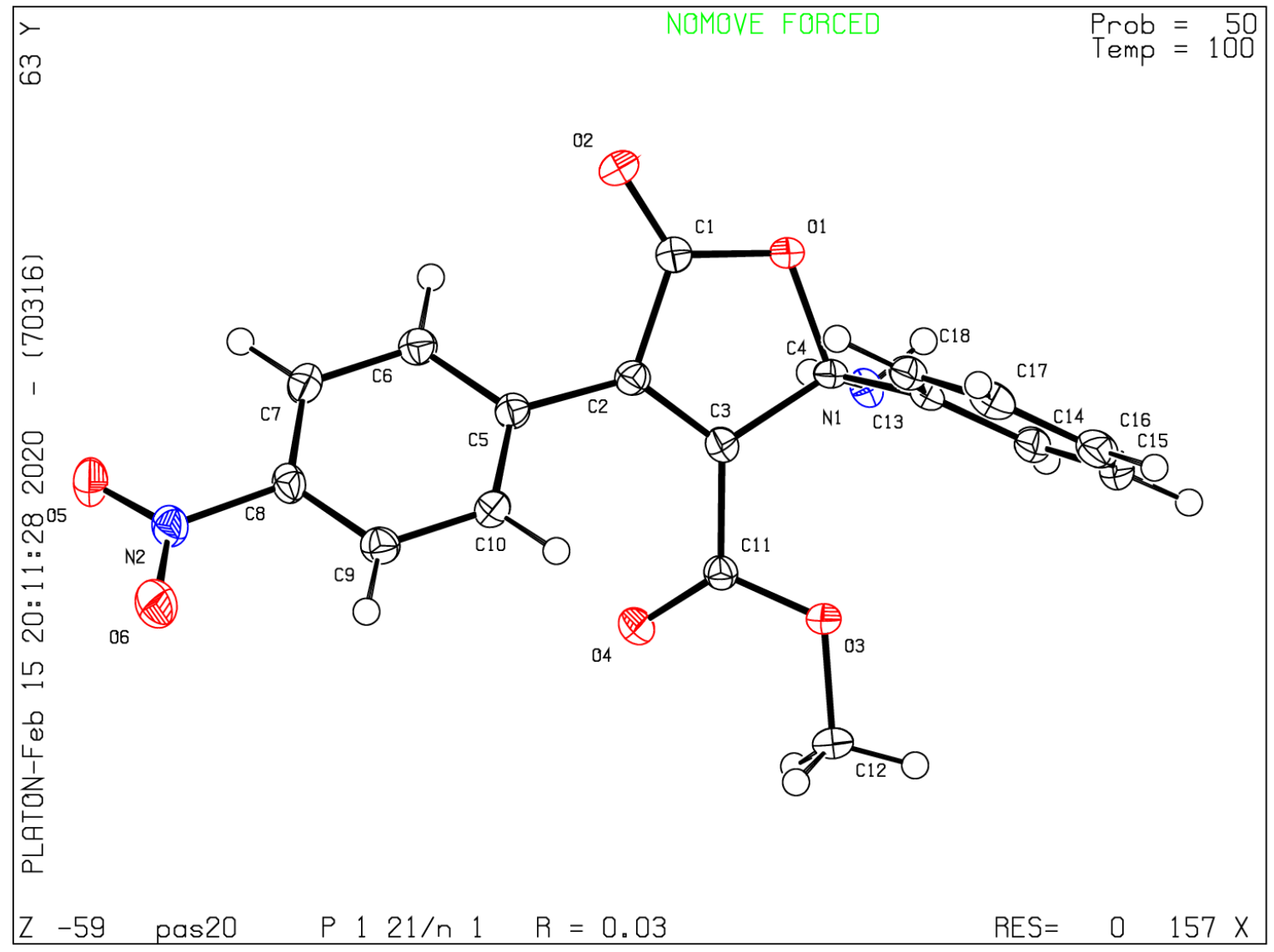


Table S3. Crystal data and structure refinement for 5a.

Empirical formula

Formula weight

Temperature/K

Crystal system

Space group

$\mathrm{a} / \AA$

$\mathrm{b} / \AA$

$\mathrm{c} / \AA$

$\alpha /{ }^{\circ}$

$\beta /{ }^{\circ}$

$\gamma /{ }^{\circ}$

Volume $/ \AA^{3}$

$\mathrm{Z}$

$\rho_{\text {calc }} \mathrm{g} / \mathrm{cm}^{3}$

$\mu / \mathrm{mm}^{-1}$

$\mathrm{F}(000)$

Crystal size $/ \mathrm{mm}^{3}$

Radiation

$2 \Theta$ range for data collection ${ }^{\circ}$

Index ranges

Reflections collected

Independent reflections

Data/restraints/parameters

Goodness-of-fit on $\mathrm{F}^{2}$

Final $\mathrm{R}$ indexes $[\mathrm{I}>=2 \sigma(\mathrm{I})]$

Final $\mathrm{R}$ indexes [all data]

Largest diff. peak/hole / e $\AA^{-3}$
$\mathrm{C}_{18} \mathrm{H}_{14} \mathrm{~N}_{2} \mathrm{O}_{6}$

354.31

100.01(10)

monoclinic

$\mathrm{P} 2{ }_{1} / \mathrm{n}$

$11.4574(3)$

$12.0859(3)$

$11.6260(3)$

90

97.433(2)

90

1596.36(7)

4

1.474

0.952

736.0

$0.23 \times 0.17 \times 0.12$

$\mathrm{CuK} \alpha(\lambda=1.54184)$

10.2 to 144.982

$-14 \leq \mathrm{h} \leq 14,-10 \leq \mathrm{k} \leq 14,-14 \leq 1 \leq 14$

7277

$3162\left[\mathrm{R}_{\mathrm{int}}=0.0195, \mathrm{R}_{\mathrm{sigma}}=0.0211\right]$

$3162 / 0 / 237$

1.025

$\mathrm{R}_{1}=0.0344, \mathrm{wR}_{2}=0.0873$

$\mathrm{R}_{1}=0.0380, \mathrm{wR}_{2}=0.0908$

$0.34 /-0.21$ 


\section{Computation details}

All calculations were performed by using the Gaussian 09 suite of quantum chemical programs. ${ }^{12}$ Geometry optimizations of compounds $7^{\mathrm{Ph}}$, syn-9 $\mathbf{9}^{\mathrm{Ph}}$, syn-9 $\mathbf{9}^{\mathrm{Ph}}$ (conf-2), syn-9 $\mathbf{9}^{\mathrm{Ph}}$ (conf3), anti-9 ${ }^{\mathrm{Ph}}$, and anti-9 ${ }^{\mathrm{Ph}}$ (conf-2) were performed at the DFT B3LYP/6-31+G(d,p) level using PCM model for acetonitrile.

Table S4. Relative stability of potassium enolates $9^{\mathrm{Ph}}(\Delta \mathrm{G}, \mathrm{kcal} / \mathrm{mol})$

(conf-2)


Table S5. Energies (au) and Cartesian Coordinates of Stationary Points for Enolates $7^{\mathrm{Ph}}$, syn-9 $\mathbf{9}^{\mathrm{Ph}}$,

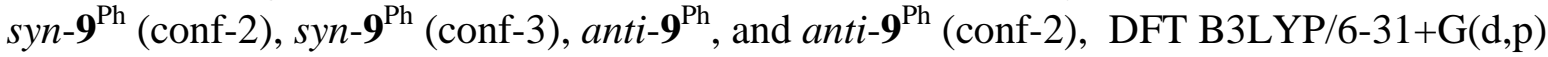
(PCM, MeCN)

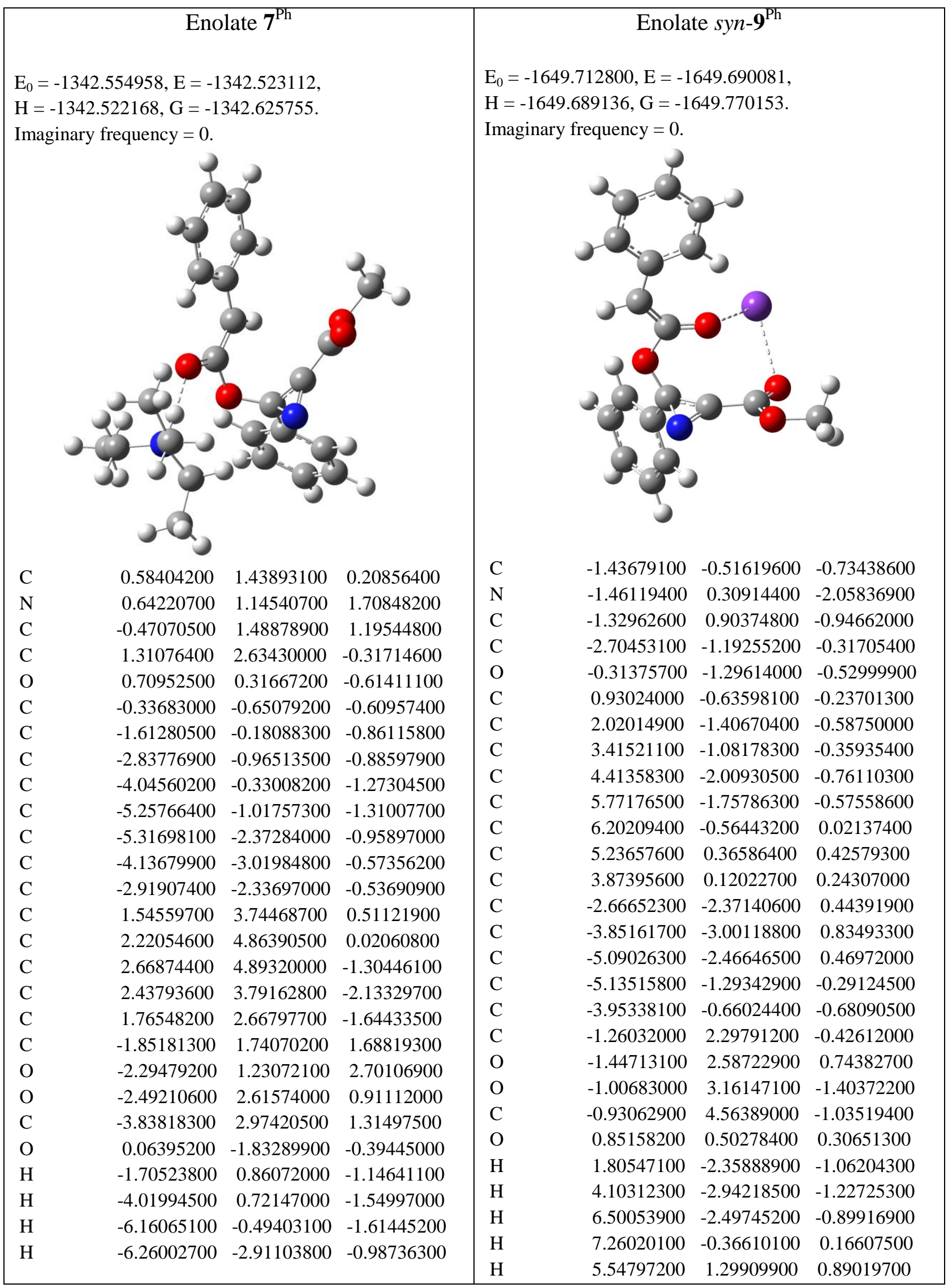




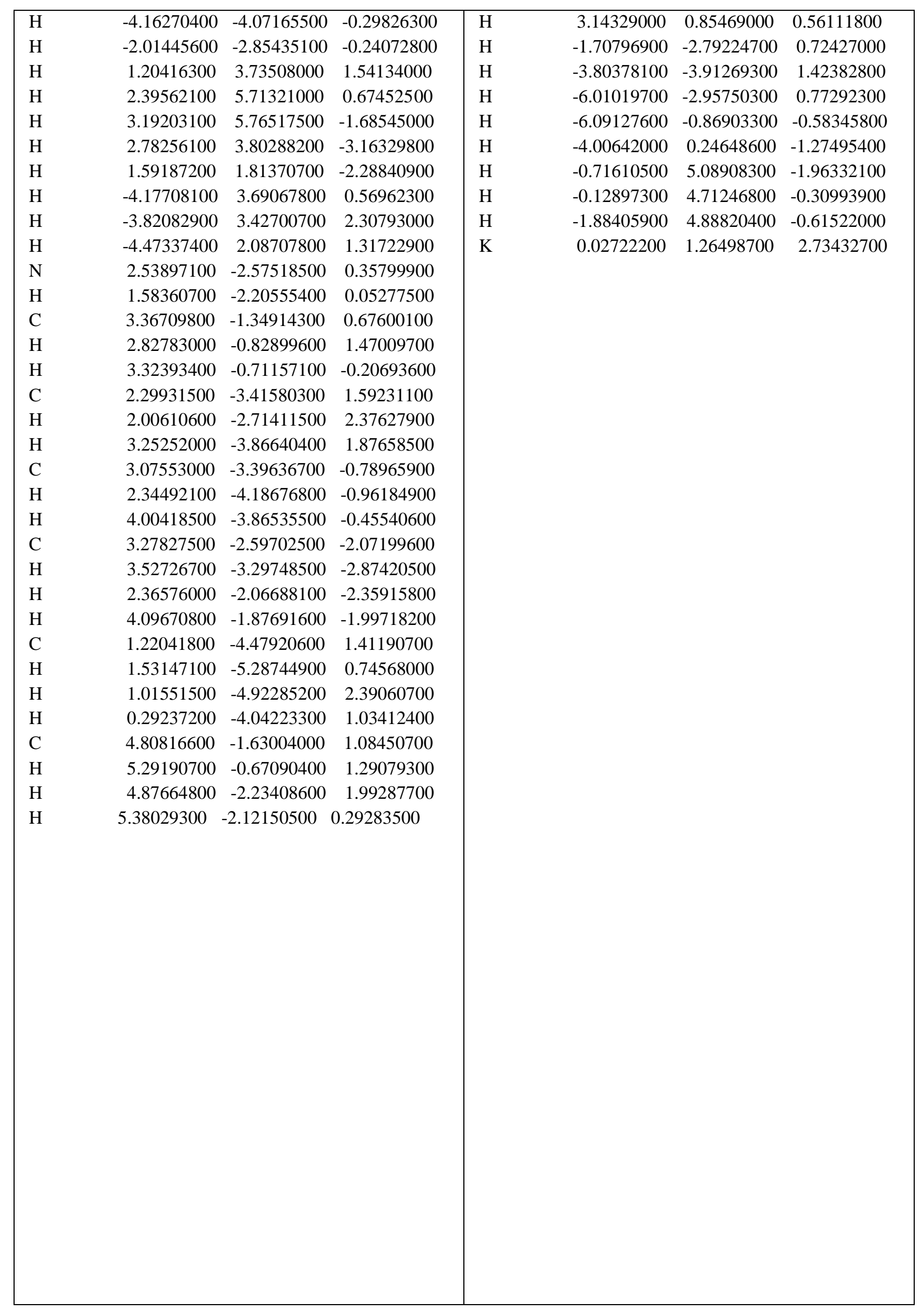




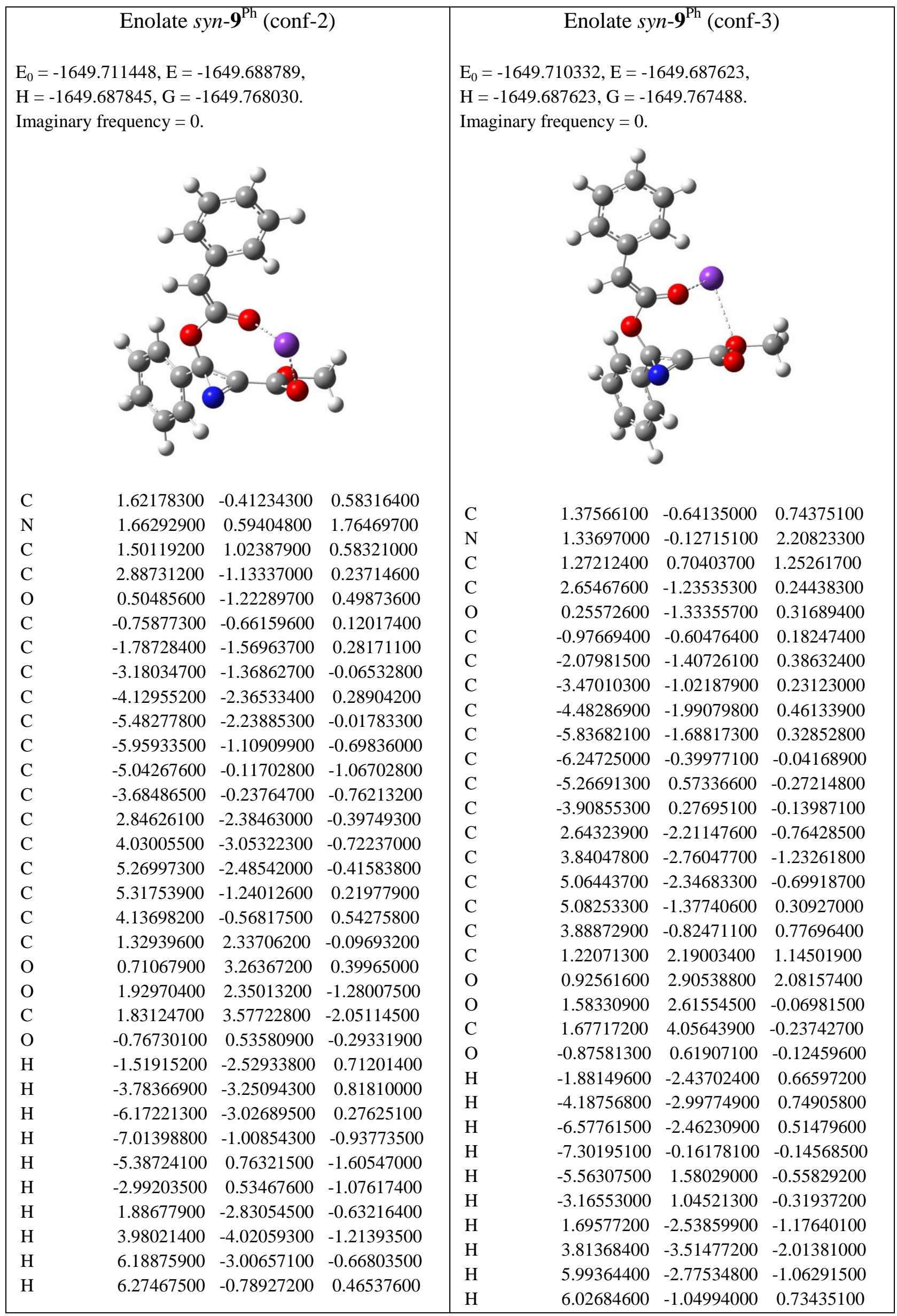




\begin{tabular}{|c|c|c|c|c|c|c|c|}
\hline $\mathrm{H}$ & 4.19057900 & 0.39585000 & 1.03815200 & $\mathrm{H}$ & 3.92010300 & -0.07671800 & 1.56257500 \\
\hline $\mathrm{H}$ & 2.39211400 & 3.38380300 & -2.96278700 & $\mathrm{H}$ & 2.03254700 & 4.20232900 & -1.25559100 \\
\hline $\mathrm{H}$ & 2.27113600 & 4.40381000 & -1.49105400 & $\mathrm{H}$ & 2.38686800 & 4.46682400 & 0.48241800 \\
\hline $\mathrm{H}$ & 0.78422900 & 3.78724100 & -2.27543700 & $\mathrm{H}$ & 0.69518000 & 4.51160000 & -0.09796800 \\
\hline $\mathrm{K}$ & -1.90742000 & 2.50861500 & 1.22236600 & $\mathrm{~K}$ & -0.05586100 & 1.52121100 & -2.48511900 \\
\hline \multicolumn{4}{|c|}{ Enolate anti-9 $^{\mathrm{Ph}}$} & \multicolumn{4}{|c|}{ Enolate anti-9 $\mathbf{9}^{\mathrm{Ph}}$ (conf-2) } \\
\hline \multicolumn{4}{|c|}{$\begin{array}{l}E_{0}=-1649.710037, E=-1649.687295 \\
H=-1649.686351, G=-1649.767265 \\
\text { Imaginary frequency }=0\end{array}$} & \multicolumn{4}{|c|}{$\begin{array}{l}E_{0}=-1649.709940, E=-1649.687232 \\
H=-1649.686288, G=-1649.766779 \\
\text { Imaginary frequency }=0\end{array}$} \\
\hline $\mathrm{C}$ & 142140600 & 000744000 & 033050600 & & 146260200 & & \\
\hline $\mathrm{N}$ & -1.29163900 & 0.04820600 & 1.86200600 & $\mathrm{~N}$ & -1.29686000 & -0.24270700 & 1.78151400 \\
\hline $\mathrm{C}$ & -0.59552900 & -0.75201000 & 1.15701600 & $\mathrm{C}$ & -0.59573400 & -0.91964100 & 0.96080900 \\
\hline $\mathrm{C}$ & -2.72746100 & -0.32395400 & -0.27372700 & $\mathrm{C}$ & -2.76124700 & -0.34901500 & -0.36681100 \\
\hline $\mathrm{O}$ & -0.88057100 & 1.23225500 & -0.26182100 & $\mathrm{O}$ & -0.94368000 & 1.23135800 & -0.16270500 \\
\hline C & 0.55447100 & 1.40763300 & -0.21284900 & $\mathrm{C}$ & 0.49024900 & 1.40805300 & -0.17103200 \\
\hline $\mathrm{C}$ & 1.30120500 & 0.33913000 & -0.68738200 & $\mathrm{C}$ & 1.21465100 & 0.40110800 & -0.79363400 \\
\hline $\mathrm{C}$ & 2.75145600 & 0.25830000 & -0.72311900 & $\mathrm{C}$ & 2.66114400 & 0.33135400 & -0.90546000 \\
\hline $\mathrm{C}$ & 3.36059500 & -0.85836600 & -1.35231000 & $\mathrm{C}$ & 3.23916900 & -0.71075000 & -1.67636000 \\
\hline $\mathrm{C}$ & 4.74561100 & -1.00307800 & -1.41186900 & $\mathrm{C}$ & 4.62003900 & -0.84354400 & -1.81358900 \\
\hline $\mathrm{C}$ & 5.58710000 & -0.03781900 & -0.84231900 & $\mathrm{C}$ & 5.48793200 & 0.05961900 & -1.18518500 \\
\hline $\mathrm{C}$ & 5.00792800 & 1.07274300 & -0.21647300 & $\mathrm{C}$ & 4.93948500 & 1.09622700 & -0.41976100 \\
\hline $\mathrm{C}$ & 3.62108000 & 1.22516100 & -0.15616000 & $\mathrm{C}$ & 3.55726300 & 1.23612300 & -0.27980500 \\
\hline $\mathrm{C}$ & -3.14623600 & 0.21063000 & -1.50184400 & $\mathrm{C}$ & -3.18615800 & 0.31296600 & -1.52882700 \\
\hline $\mathrm{C}$ & -4.35942000 & -0.18826800 & -2.07048800 & $\mathrm{C}$ & -4.39760500 & -0.03017700 & -2.13595200 \\
\hline C & -5.17170100 & -1.12142200 & -1.42014900 & $\mathrm{C}$ & -5.20242900 & -1.03344500 & -1.58885900 \\
\hline $\mathrm{C}$ & -4.76048900 & -1.65619600 & -0.19426300 & $\mathrm{C}$ & -4.78570000 & -1.69433800 & -0.42808900 \\
\hline $\mathrm{C}$ & -3.54807000 & -1.26262500 & 0.37462600 & $\mathrm{C}$ & -3.57426200 & -1.35709600 & 0.17822500 \\
\hline $\mathrm{C}$ & 0.37324000 & -1.85012900 & 1.41530400 & $\mathrm{C}$ & 0.35218700 & -2.06247200 & 0.96865300 \\
\hline $\mathrm{O}$ & 1.03703300 & -1.91969500 & 2.43402400 & $\mathrm{O}$ & 0.42754300 & -2.87493700 & 0.06431400 \\
\hline $\mathrm{O}$ & 0.36763600 & -2.74669200 & 0.42664100 & $\mathrm{O}$ & 1.04809500 & -2.09894100 & 2.10822500 \\
\hline $\mathrm{C}$ & 1.24147200 & -3.89233100 & 0.58924000 & $\mathrm{C}$ & 1.98034000 & -3.19953300 & 2.26250300 \\
\hline $\mathrm{O}$ & 0.89931400 & 2.52445400 & 0.24127900 & $\mathrm{O}$ & 0.85725000 & 2.47273700 & 0.38077000 \\
\hline $\mathrm{H}$ & 0.76131300 & -0.47231000 & -1.16105900 & $\mathrm{H}$ & 0.65439900 & -0.35724700 & -1.32770000 \\
\hline $\mathrm{H}$ & 2.72489400 & -1.61818300 & -1.80147800 & $\mathrm{H}$ & 2.58259300 & -1.42152500 & -2.17312200 \\
\hline $\mathrm{H}$ & 5.17157200 & -1.87333900 & -1.90533600 & $\mathrm{H}$ & 5.02174000 & -1.65564800 & -2.41477900 \\
\hline $\mathrm{H}$ & 6.66669200 & -0.14844800 & -0.88755400 & $\mathrm{H}$ & 6.56417700 & -0.04127100 & -1.29170900 \\
\hline $\mathrm{H}$ & 5.64412300 & 1.83342900 & 0.23023500 & $\mathrm{H}$ & 5.59656200 & 1.80802500 & 0.07478000 \\
\hline $\mathrm{H}$ & 3.19007900 & 2.09442200 & 0.32632400 & $\mathrm{H}$ & 3.14986000 & 2.04752300 & 0.31177000 \\
\hline $\mathrm{H}$ & -2.52001900 & 0.93682100 & -2.00711300 & $\mathrm{H}$ & -2.56554300 & 1.09420200 & -1.95239800 \\
\hline $\mathrm{H}$ & -4.66876800 & 0.23394300 & -3.02227300 & $\mathrm{H}$ & -4.71162500 & 0.49017300 & -3.03619200 \\
\hline $\mathrm{H}$ & -6.11444700 & -1.42949700 & -1.86245400 & $\mathrm{H}$ & -6.14394100 & -1.29849200 & -2.06067500 \\
\hline $\mathrm{H}$ & -5.38365700 & -2.38134400 & 0.32093800 & $\mathrm{H}$ & -5.40363700 & -2.47448900 & 0.00680000 \\
\hline
\end{tabular}




\begin{tabular}{|lrrr|rrrr|}
\hline $\mathrm{H}$ & -3.24213800 & -1.68469800 & 1.32649100 & $\mathrm{H}$ & -3.26264300 & -1.87722000 & 1.07834100 \\
$\mathrm{H}$ & 1.09189600 & -4.49314700 & -0.30545700 & $\mathrm{H}$ & 2.45169100 & -3.03881800 & 3.22994300 \\
$\mathrm{H}$ & 0.95926200 & -4.45201600 & 1.48285500 & $\mathrm{H}$ & 2.72095100 & -3.17557500 & 1.46154300 \\
$\mathrm{H}$ & 2.27839600 & -3.56136300 & 0.66517200 & $\mathrm{H}$ & 1.44096600 & -4.14832700 & 2.24706300 \\
$\mathrm{~K}$ & -1.35411000 & 3.89298300 & 0.84045800 & $\mathrm{~K}$ & -1.34660000 & 3.68227700 & 1.37371000 \\
\hline
\end{tabular}




\section{References}

1. Rostovskii, N. V.; Agafonova, A. V.; Smetanin, I. A.; Novikov, M. S.; Khlebnikov, A. F.; Ruvinskaya, J. O.; Starova, G. L. Metal-Catalyzed Isomerization of 5-HeteroatomSubstituted Isoxazoles as a New Route to 2-Halo- $2 H$-azirines. Synthesis 2017, 49, 4478-4488.

2. Agafonova, A. V.; Rostovskii, N. V.; Smetanin, I. A.; Starova, G. L.; Khlebnikov, A. F.; Novikov, M. S. Synthesis of 2-(Di/tri/tetraazolyl)-2H-azirine-2-carboxylates by Halogen Substitution: Evidence for an $\mathrm{S}_{\mathrm{N}} 2^{\prime}-\mathrm{S}_{\mathrm{N}} 2^{\prime}$ Cascade Mechanism. J. Org. Chem. 2018, 83, 13473-13480.

3. Agafonova, A. V.; Smetanin, I. A.; Rostovskii, N. V.; Khlebnikov, A. F.; Novikov, M. S. Easy Access to 2-Fluoro- and 2-Iodo-2H-azirines via the Halex Reaction. Synthesis 2019, $51,4582-4589$.

4. Pinho e Melo, T. M. V. D; Lopes, C. S. J.; Cardoso, A. L.; Rocha Gonsalves, A. M. d'A. Synthesis of 2-Halo-2H-azirines. Tetrahedron 2001, 57, 6203-6208.

5. Agafonova, A. V.; Smetanin, I. A.; Rostovskii, N. V.; Khlebnikov, A. F.; Novikov, M. S. Synthesis of 2-Halo-2H-azirine-2-carboxylic Acid Amides and Esters by Isomerization of 5-(Dialkylamino/alkoxy)-Substituted Isoxazoles, Catalyzed by Iron(II) Sulfate. Chemistry of Heterocyclic Compounds 2017, 53, 1068-1071.

6. Rostovskii, N. V.; Smetanin, I. A.; Agafonova, A. V.; Sakharov, P. A.; Ruvinskaya, J. O.; Khlebnikov, A. F.; Novikov, M. S. Facile Access to 2-Acyloxy-, Aryloxy- and Alkenyloxy-2H-azirines via an $\mathrm{S}_{\mathrm{N}} 2{ }^{\prime}-\mathrm{S}_{\mathrm{N}} 2$ ' Cascade in 2-Halo-2H-azirines. Org. Biomol. Chem. 2018, 16, 3248-3257.

7. Smetanin, I. A.; Novikov, M. S.; Agafonova, A. V.; Rostovskii, N. V.; Khlebnikov, A. F.; Kudryavtsev, I. V.; Terpilowski, M. A.; Serebriakova, M. K.; Trulioff, A. S.; Goncharov N. V. A Novel Strategy for the Synthesis of Thermally Stable and Apoptosis-Inducing 2,3-Dihydroazetes. Org. Biomol. Chem. 2016, 14, 4479-4487.

8. Dolomanov, O.V.; Bourhis, L.J.; Gildea, R.J; Howard, J.A.K.; Puschmann, H. OLEX2: a complete structure solution, refinement and analysis program. J. Appl. Cryst. 2009, 42, 339-341.

9. Palatinus, L.; Chapuis, G. SUPERFLIP - a computer program for the solution of crystal structures by charge flipping in arbitrary dimensions. J. Appl. Cryst. 2007, 40, 786-790; Palatinus, L.; van der Lee, A. Symmetry determination following structure solution in P1. J. Appl. Cryst. 2008, 41, 975-984; Palatinus, L.; Prathapa, S. J.; van Smaalen, S. EDMA: a computer program for topological analysis of discrete electron densities. J. Appl. Cryst. 2012, 45, 575-580.

10. Sheldrick, G.M. Crystal structure refinement with SHELXL. Acta Cryst. 2015, C71, 3-8.

11. Sheldrick, G.M. SHELXT - Integrated space-group and crystal-structure determination. Acta Cryst. 2015, A71, 3-8.

12. Gaussian 09, Revision D.01, Frisch, M. J.; Trucks, G.W.; Schlegel, H. B.; Scuseria, G. E.; Robb, M. A.; Cheeseman, J. R.; Scalmani, G.; Barone, V.; Mennucci, B.; Petersson, G. A.; Nakatsuji, H.; Caricato, M.; Li, X.; Hratchian, H. P.; Izmaylov, A. F.; Bloino, J.; 
Zheng, G.; Sonnenberg, J. L.; Hada, M.; Ehara, M.; Toyota, K.; Fukuda, R.; Hasegawa, J.; Ishida, M.; Nakajima, T.; Honda, Y.; Kitao, O.; Nakai, H.; Vreven, T.; Montgomery, J. A.; Peralta, J. E.; Ogliaro, F.; Bearpark, M.; Heyd, J. J.; Brothers, E.; Kudin, K. N.; Staroverov, V. N.; Kobayashi, R.; Normand, J.; Raghavachari, K.; Rendell, A.; Burant, J. C.; Iyengar, S. S.; Tomasi, J.; Cossi, M.; Rega, N.; Millam, N. J.; Klene, M.; Knox, J. E.; Cross, J. B.; Bakken, V.; Adamo, C.; Jaramillo, J.; Gomperts, R.; Stratmann, R. E.; Yazyev, O.; Austin, A. J.; Cammi, R.; Pomelli, C.; Ochterski, J. W.; Martin, R. L.; Morokuma, K.; Zakrzewski, V. G.; Voth, G. A.; Salvador, P.; Dannenberg, J. J.; Dapprich, S.; Daniels, A. D.; Farkas, Ö.; Foresman, J. B.; Ortiz, J. V.; Cioslowski, J.; Fox, D. J. Gaussian 09, Revision C.01; Gaussian: Wallingford CT, 2013. 